\title{
EMDR Therapy Overview
}

The world is round and the place which may seem like the end may also be the beginning.-Ivy Baker Priest (Parade, 1958)

\section{REINTRODUCTION TO EMDR THERAPY}

The goal of EMDR is to achieve the most profound and comprehensive treatment effects possible in the shortest period of time, while maintaining client stability within a balanced system.

(SHAPIRO, 2018, P. 6)

This chapter summarizes the information covered in the most recent EMDR therapy trainings, as well as Dr. Francine Shapiro's primary text (2018), in the hope of providing additional clarity to the newly trained clinician. It looks at different ways trauma can be conceptualized and includes a reintroduction to the Adaptive Information Processing (AIP) model, the concept of the three-pronged approach, targets associated with EMDR therapy, and clinical guidelines pertinent to EMDR therapy. References to educational learning materials, research, other relevant supplementary information, and key points that are important to remember during the EMDR therapy learning process are also covered.

Although the EMDR therapy principles, protocols, and procedures have been simplified with tables and figures in this Primer, it is not a mechanistic or cookie-cutter approach. EMDR therapy is a fluid process, and the results will vary from client to client. Formal training in EMDR therapy allows clinicians to initiate understanding its model, methodology, and mechanism. This knowledge, combined with their own clinical intuition, allows them to begin practicing this therapeutic approach. No one should read this book thinking that it is a substitute for formal training. EMDR therapy seems simple on its surface; however, in reality, its competent execution is complex and complicated. 
Extensive familiarity with Dr. Shapiro's primary text is a prerequisite for the reading of this Primer, which is intended to supplement, not replace, her required pre-training readings. No clinician who intends to utilize EMDR therapy with clients can afford to be without Eye Movement Desensitization and Reprocessing: Basic Principles, Protocols, and Procedures (Shapiro, 2018). In the early days of implementation, you may need to refer to Dr. Shapiro's book daily. Read it often and use it as your primary EMDR therapy reference guide. Every time you read it, you will probably notice something that you did not quite understand or retain the first few times around. Read it thoroughly and refer to it often. It is not necessary that you memorize the book; just remember that it is there for you as an ongoing guide to your clinical work. It is also suggested that clinicians read Getting Past Your Past (Shapiro, 2012b). This book provides clinicians and clients alike a greater understanding of why people act the way they do. Clinicians may also read EMDR: The Breakthrough Therapy for Overcoming Anxiety, Stress, and Trauma (Shapiro \& Forrest, 2016) as a basic overview of the clinical applications of EMDR therapy.

EMDR therapy, a reprocessing therapy, is a robust, comprehensive psychotherapeutic treatment approach comprising eight distinct phases that begin with the clinician's initial contact with the client. These include taking a thorough client history, preparing the client for the EMDR therapy process, setting up the protocol, desensitizing and reprocessing the trauma, installing a positive cognition (PC), doing a body scan to check for residual trauma, "closing down" a session, and reevaluating the status of a trauma. All eight phases must be in place in the order described previously. Chapter 2 contains an in-depth discussion of these phases.

There have been other offshoots of EMDR therapy since its inception (e.g., Grand and Goldberg, 2011; Kip et al., 2013; Pace, 2003). These techniques have their supporters and many successes may have been reported, but these treatments, to date, have little to no validation in the research literature. The efficacy of many of these models has not been tested within a scientific, empirical setting, whereas EMDR therapy's validity has been proven repeatedly.

\section{TRAUMA}

\section{WHAT IS TRAUMA?}

The diagnostic criteria for posttraumatic stress disorder (PTSD; DSM-V, 309.81; ICD-10, F43.10) cited in the Diagnostic and Satistical Manual of Mental Disorders (5th ed.; DSM-5; American Psychiatric Association [APA], 2013) is the definition used most frequently to describe acute trauma in adults. This definition describes trauma as an event experienced or witnessed by a person that results in intrusive symptoms, avoidance, negative alterations in cognitions and mood, and alterations in arousal and reactivity (APA, 2013). Flannery describes trauma as

the state of severe fright that we experience when we are confronted with a sudden, unexpected, potentially life-threatening event, over which we have no control, and to which we are unable to respond effectively no matter how hard we try (1995).

A child who was sexually abused by her older brother may grow up to believe "I am bad" or "The world is unsafe." When an individual experiences a traumatic event, the event can become entrenched (or fixed) in the form of irrational beliefs, negative emotions, blocked energy, and/or physical symptoms, such as anxieties, phobias, flashbacks, nightmares, and/ or fears. Regardless of the magnitude of the trauma, it may have the potential for negatively impacting an individual's self-confidence and self-efficacy. The event can become locked or 
"stuck" in the memory network (i.e., "an associated system of information" [Shapiro, 2018, p. 30]) in its original form, causing an array of traumatic or PTSD symptoms. Triggers activate images, physical sensations, tastes, smells, sounds, and beliefs that might echo the experience as though it were the day it originally happened or cause other distortions in perception of current events. Reminders of the event have the potential for triggering an emotional or physical response. By utilizing EMDR therapy, the client can unblock the traumatic information and can fully experience and integrate the trauma toward a healthy resolution.

\section{TYPES OF TRAUMA}

Dr. Shapiro (2018) distinguishes between big “T” traumas and other adverse life experiences or disturbing life events (formerly referred to as small " $t$ " traumas). When a person hears the word "trauma," experiences and images of man-made events such as fires, explosions, automobile accidents, or natural disasters, which include hurricanes, floods, and tornadoes, emerge. Sexual abuse, a massive heart attack, death of a loved one, Hurricane Katrina, and the 9/11 attacks on the World Trade Center by international terrorists are graphic examples of big " $T$ " traumas. Among other descriptors, these types of traumas can be defined as dangerous and life threatening and fit the criteria in the DSM-5 (APA, 2013) as stated previously.

Then there are the traumas Shapiro (2018) has designated as adverse life experiences. These types of events may be subtler and tend to impact one's beliefs about self, others, and the world. Adverse life experiences are those that can affect our sense of self, self-esteem, selfdefinition, self-confidence, and optimal behavior. They influence how we see ourselves as a part of the bigger whole. They are often ubiquitous (i.e., constantly encountered) in nature and are stored in state-dependent mode in our memory network. Unless persistent throughout the client's childhood, adverse life experiences usually do not have much impact on overall development, yet maintain the ability to elicit negative self-attributions and have potential for other long-term negative consequences. The clinical presentations that often signal the presence of past adverse life events may be low self-esteem and anxiety as well as panic disorders and/or phobias, depression, posttraumatic symptoms, and the presence of dissociative disorders. The domains of dysfunction tend to be emotional, somatic, cognitive, and relational.

To illustrate the difference between an adverse life experience and a big " $\mathrm{T}$ " trauma, let us consider the case of Rebecca, who grew up as "the minister's daughter." As the offspring of a local pastor, Rebecca grew up, figuratively speaking, in a glass house. She believed that her father's job rested on her behavior inside and outside of her home. In her world, everyone was watching. She was always in the spotlight, and no one seemed to want to share his or her life with her. She went through childhood with few friends. "I remember before and after church, the groups of kids forming. I was the outsider. No one invited me in.” All the kids were afraid that every move they made would be reported to her daddy. Being at home was not much better. Her father was never home. He was always out "tending to his flock" and had little time left for his own family. Her mother was not of much comfort either, because she spent much of her time trying to be perfect as well. Living in a glass house was not easy for any of them, especially Rebecca, the oldest of three. By the time Rebecca entered therapy, she was a wife and mother. She thought she had to be perfect in motherhood and in her marriage as well. She became frustrated, angry, and lonely. She felt misunderstood and neglected by her husband. He was never there. He never listened. She thought she could do nothing right, as hard as she tried.

Probing into Rebecca's earliest childhood memories, no tragic or traumatic memories (i.e., big " $\mathrm{T}$ ” traumas) emerged. As she continued to explore her past, the hardships and rigors of living in a glass house as the preacher's daughter slowly became apparent. The original 
target that initiated a round of EMDR reprocessing sessions focused on Rebecca "sitting on my hands in church and being a good little girl." Her negative belief about herself as she focused on this global touchstone event was "I have to be perfect." She felt isolated, overlooked, and abandoned by her parents and the parishioners of her father's church. These were undoubtedly adverse life experiences. There was no one single event or series of traumatic events that set her current problem or issue in place. It was her way of life; and how, where, and why she was forced to live as a child caused a specific set of symptoms and interfered with her living happily and successfully in the present.

The differentiation between adverse life experiences and big " $\mathrm{T}$ " traumas often appears too simplistic. Another way of discussing the types of trauma is to look at it in terms of shock or developmental trauma.

Shock trauma involves a sudden threat that is perceived by the central nervous system as overwhelming and/or life threatening. It is a single-episode traumatic event. Examples include car accidents, violence, surgery, hurricanes and other natural disasters, rape, battlefield assaults, and war.

Developmental trauma refers to events that occur over time and gradually affect and alter a client's neurological system to the point that it remains in a traumatic state. This type of trauma may cause interruptions in a child's natural psychological growth. Examples of developmental trauma are abandonment or long-term separation from a parent, an unstable or unsafe environment, neglect, serious illness, physical or sexual abuse, and betrayal at the hands of a caregiver. This type of trauma can have a negative impact on a child's sense of safety and security in the world and tends to set the stage for future trauma in adulthood as the sense of fear and helplessness that accompany it goes unresolved.

Table 1.1 outlines more definitely the differences between big " $\mathrm{T}$ ” traumas and adverse life experiences or disturbing life events (i.e., small " $\mathrm{t}$ ” traumas).

\section{ADAPTIVE INFORMATION PROCESSING—“THE PAST DRIVES THE PRESENT"}

EMDR therapy is a distinct integrative psychotherapeutic approach and is compatible with other major orientations of psychotherapy. This eight-phase approach is led by an information processing model and guides clinical practice (i.e., case conceptualization and treatment planning) in general.

Dr. Francine Shapiro developed a hypothetical information processing model of learning called the AIP model (changed from Accelerated Information Processing model in 1995) to provide a theoretical framework and principles for EMDR therapy. Accelerated Information Processing clarifies how EMDR therapy works, and AIP guides how it is used (Table 1.2). Dr. Shapiro recognized the need to more efficiently explain the consistent treatment effects being obtained and reported from EMDR therapy.

AIP elaborates on the observed treatment effects of EMDR therapy by describing an innate physiological system that helps to transform disturbing information into adaptive resolution by psychologically integrating the information. In this model, memory networks constitute the basis of our perceptions, attitudes, and behaviors and constitute the basis of health or dysfunction. They may contain positive and negative experiences and may be adaptive or maladaptive based on the nature of these experiences. These memories consist of stored information, such as sensory input (i.e., captured by our five senses), thoughts, emotions, and beliefs (Figure 1.1). Dr. Shapiro believes that disturbing events, whether big " $T$ " 


\section{TABLE 1.1 Similarities and Differences Between Big "T" and Small}

"t" Trauma

BIG "T"TRAUMA

SMALL "t"TRAUMA (DISTURBING/

DISTRESSING LIFE EVENTS)

Major event normally seen as traumatic

May be a single- or multiple-event trauma

May be pervasive

Most often there is intrusive imagery

Still elicits similar negative beliefs, emotions, and physical sensations

Lasting negative effect on the client's sense of safety in the world
Disturbing/distressing life event that may not always be perceived as traumatic

More common and ubiquitous; usually accumulates over time from childhood

More often it is pervasive and ongoing

Often there is no intrusive imagery

Still elicits similar negative beliefs, emotions, and physical sensations

Lasting negative effect on the client's sense of self (self-confidence, self-esteem, selfdefinition)

\section{Examples:}

Serious accidents (e.g., automobile or bike accidents, plane crashes, serious falls)

Natural disasters (e.g., earthquakes, tornadoes, tsunamis, forest fires, volcanic eruptions, floods)

Man-made disasters (e.g., 9/11, explosions, fires, wars, acts of terrorism)

Major life changes (e.g., serious illnesses, loss of loved ones)

Physical and sexual assaults

Major surgeries, life-threatening illnesses (e.g., cancer, heart attacks, craniotomies, heart bypasses)

Ongoing life events (e.g., sexual abuse, domestic violence)

War- and combat-related incidents

\section{Examples:}

Moving multiple times during childhood

Excessive teasing or bullying

Persistent physical illnesses

Constant criticism

Rejections

Betrayals

Disparaging remarks

Losing jobs

Divorce or witnessing parental conflict

Unmet developmental needs

Death of pets

Public shaming, humiliation, or failure

Unresolved guilt

Physical or emotional neglect

Getting lost

Chronic harassment

\section{TABLE 1.2 Accelerated Information Processing Versus Adaptive} Information Processing

\section{ACCELERATED INFORMATION PROCESSING AIP (HOW IT WORKS) (HOW IT IS USED)}

Working hypothesis

Explains how EMDR works

Developed to explain the rapid manner in which clinical results are achieved

Simple desensitization treatment effect
Working model

Explains why EMDR works

Developed to explain the clinical phenomena observed 


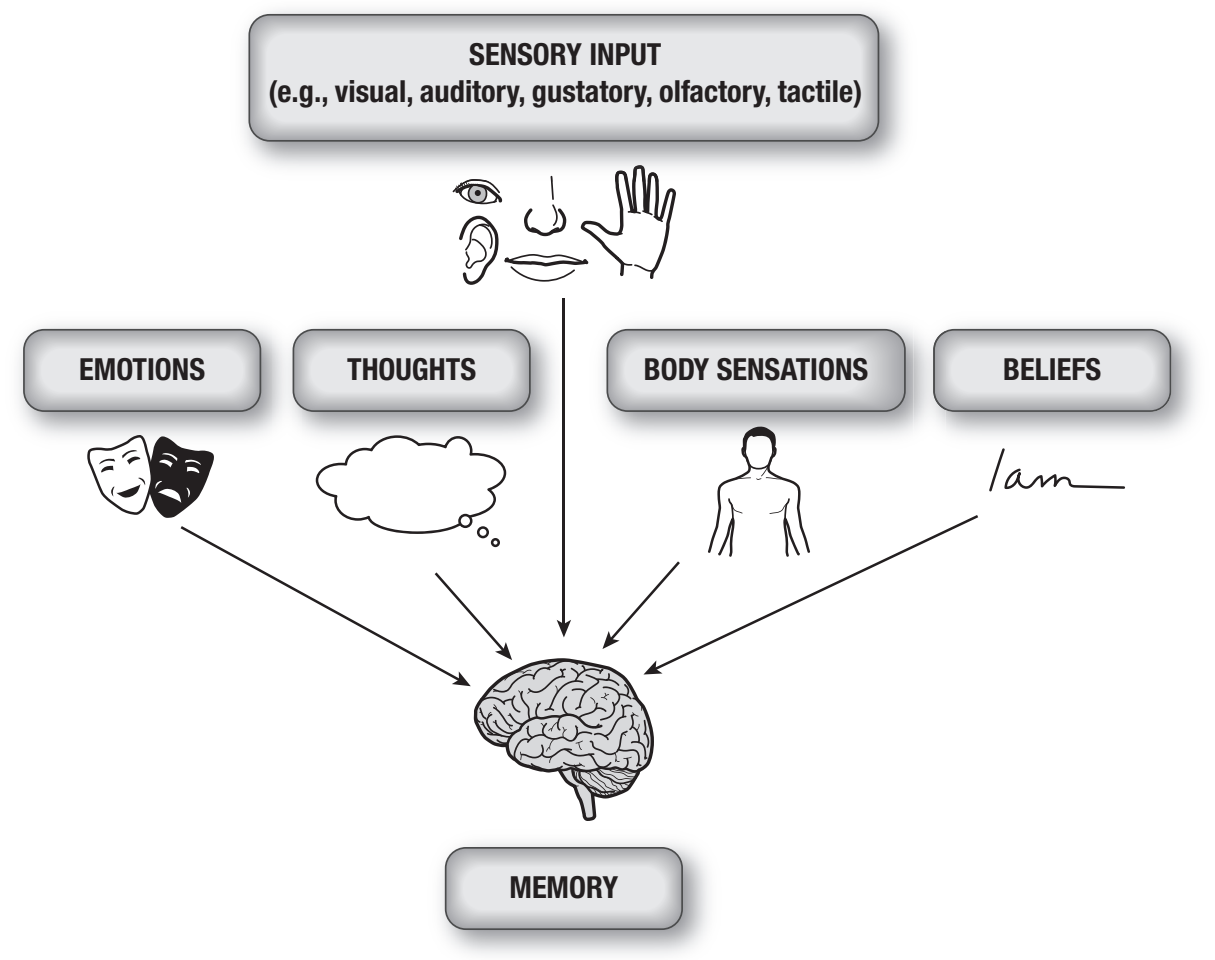

FIGURE 1.1 Diagram illustrating the components of memory.

traumas or adverse life experiences, are the primary source of our current dysfunction. When trauma happens, it causes a disruption in our information processing system, leaving any associated sights, sounds, thoughts, or feelings unprocessed and, subsequently, dysfunctionally stored as they are perceived (Shapiro, 2018). See Table 1.3 for examples of adaptive versus maladaptive resolution.

Shapiro (2018) posits that inherent in the AIP model is a psychological self-healing construct similar to the body's healing response to physical injury. For example, if you get a splinter stuck in your finger, your body's automatic response is to heal the area of injury. However, because the area is blocked by the splinter, healing cannot easily occur until the sliver is removed. In terms of mental processes, it is the inherent tendency of the information processing system to also move toward a state of health. So, even when something mildly disturbing happens, you may think about it, talk about it, and process it. You usually find that, within a day or so, you are no longer thinking so intensely about the event and, when you do, you have come to a resolution. For instance, if you are angry at your spouse, you may start to remember that your spouse has some good qualities as well as these very annoying ones. It is a case of the mind adaptively processing the disturbing material and connecting that disturbance into the larger picture of the experience. Table 1.4 demonstrates how EMDR therapy catalyzes healing and learning.

On the other hand, when a trauma occurs that is too large for your system to adequately process, it can become "stuck" (i.e., dysfunctionally stored) in the central nervous system. 


\section{TABLE 1.3 Adaptive Versus Maladaptive Resolution}

ADAPTIVE

Big “T” (e.g., safety)

I survived.

I can learn from this.

I can protect myself.

I am safe.

\section{MALADAPTIVE}

Small "t” (i.e., adverse or disturbing/distressing life events; e.g., responsibility)

I am fine as I am.

I did the best I could.

I am significant/important.

Low self-esteem

Irrational guilt

Self-neglect, co-dependence

Driving phobia

shbacks

Intense driving anxiety

Night terrors

\section{TABLE 1.4 EMDR Therapy Catalyzes Learning}

\section{TARGET}

When the target is a disturbing memory.

When the target is positive (i.e., an alternative desirable imagined future).

Negative images, beliefs, and emotions

become less vivid, less enhanced, and less valid.

Before reprocessing, links to dysfunctional material.
Positive images, beliefs, and emotions

become more vivid, more enhanced, and more valid.

Learning is a continuum.

Maladaptive responses, such as flashbacks or dreams, can be triggered by present stimuli, and there may be attempts of the information processing system to resolve the trauma (Shapiro, 2018). When the system becomes overloaded as just described, EMDR therapy is proving to be the treatment of choice for many to help restart this mental healing process and allow the traumas to be reprocessed. See Exhibit 1.1 for a graphical representation of the AIP model.

The AIP model also posits that earlier life experiences set the stage for later life strengths or problems. Information from earlier disturbing life events can be physiologically and dysfunctionally stored in the nervous system if not properly assimilated at the time of the event. Problematic behaviors and disorders can occur as a result. Table 1.5 describes the differences between dysfunctionally stored and adaptively stored memories.

At the time of disturbing or traumatic events, information may be stored in the central nervous system in state-specific form (i.e., the negative cognitive belief and emotional and physical sensations the client experienced at the time of the traumatic event remain stored in the central nervous system just as if the trauma is happening in the now). Over time, a client may develop repeated negative patterns of feeling, sensing, thinking, believing, and behaving as a result of the dysfunctionally stored material. These patterns are stimulated, activated, or triggered by stimuli in the present that cause a client to react in the same or similar ways 
EXHIBIT 1.1 Adaptive Information Processing Model: The Information Processing System at Work

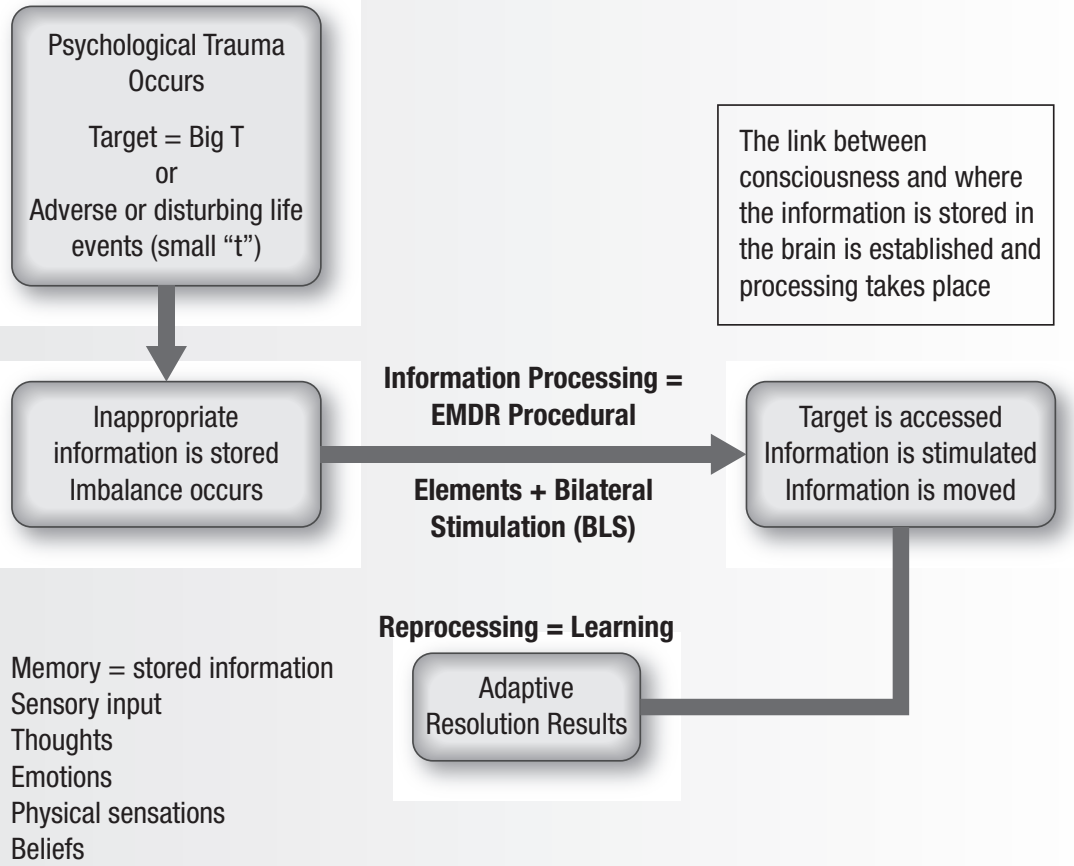

Abbreviation: BLS, bilateral stimulation.

TABLE 1.5 Differences Between Dysfunctionally Stored and Adaptively Stored Memories

\section{DYSFUNCTIONALLY STORED MEMORIES}

Information processing system is overwhelmed and becomes "stuck"

Embraces an inappropriate, developmentally arrested lack of power in the past

Past-oriented or developmentally arrested, dysfunctional perspective

Stored in incorrect form of memory (i.e., implicit/motoric)

Past is present

\section{ADAPTIVELY STORED MEMORIES}

Information processing system is able to connect to current information and resources and adequately process information

Embraces an age-appropriate power in the present

Present-oriented and age-appropriate, adaptive perspective

Stored in correct form of memory (i.e., explicit/narrative)

Past is past 


\title{
TABLE 1.6 Activation Components of EMDR Therapy
}

\section{ACCESS}

Frozen dysfunctional memory

\section{STIMULATE}

Information processing system

\section{MOVE}

Information to adaptive resolution

\author{
RESULTS \\ Lessening of disturbance \\ Gained information and insights \\ Changes in emotional and physical responses
}

as in the past. Shapiro (2018) states throughout her basic text that the negative beliefs and affect from past events spill into the present. By processing earlier traumatic memories, EMDR therapy enables the client to generalize positive affect and cognitions to associated memories found throughout the "neuro" networks (i.e., memory networks), thus allowing more appropriate behaviors in the present. Table 1.6 demonstrates a more simplified version of how EMDR reprocessing works (Shapiro, 2009-2017a, 2009-2017b).

Cognitive behavioral techniques, such as systematic desensitization, imaginal exposure, or flooding, require the client to focus on anxiety-provoking behaviors and irrational thoughts or to relive the trauma or other adverse life experiences. EMDR therapy accesses or develops adequate current resources, and then targets the experiences that caused the negative cognition, affect, and physical sensations to become "stuck" in a client's nervous system. Once the memories have been reprocessed utilizing EMDR, a physiological shift can occur that causes the disturbing picture to fade appropriately with the associated negative self-belief, feelings, and physical sensations. The "block" (i.e., dysfunctionally stored information) in the client's nervous system is shifted, and the disturbance is brought to an adaptive resolution as the natural healing process is activated. The primary byproduct of reprocessing is a decrease or elimination of the negative charge associated with the trauma or disturbing life events.

Changes in perception and attitude, experiencing moments of insight, and subtle differences in the way a person thinks, feels, behaves, and believes are byproducts as well. The changes can be immediate. Take, for instance, a session with a young woman who had been brutally raped by her ex-boyfriend. During the Assessment Phase, Andrea's terror appeared raggedly etched in her face and slumped demeanor. After many successive sets of bilateral stimulation (BLS), her pale facial features began to redden, her posture began to straighten, and her breath began to gain strength and resolve as she spontaneously stated, "He took my power that night. No more! I am taking my power back. He no longer has the 


\section{EXHIBIT 1.2 Adaptive Information Processing Model: Information Processing Mechanism}

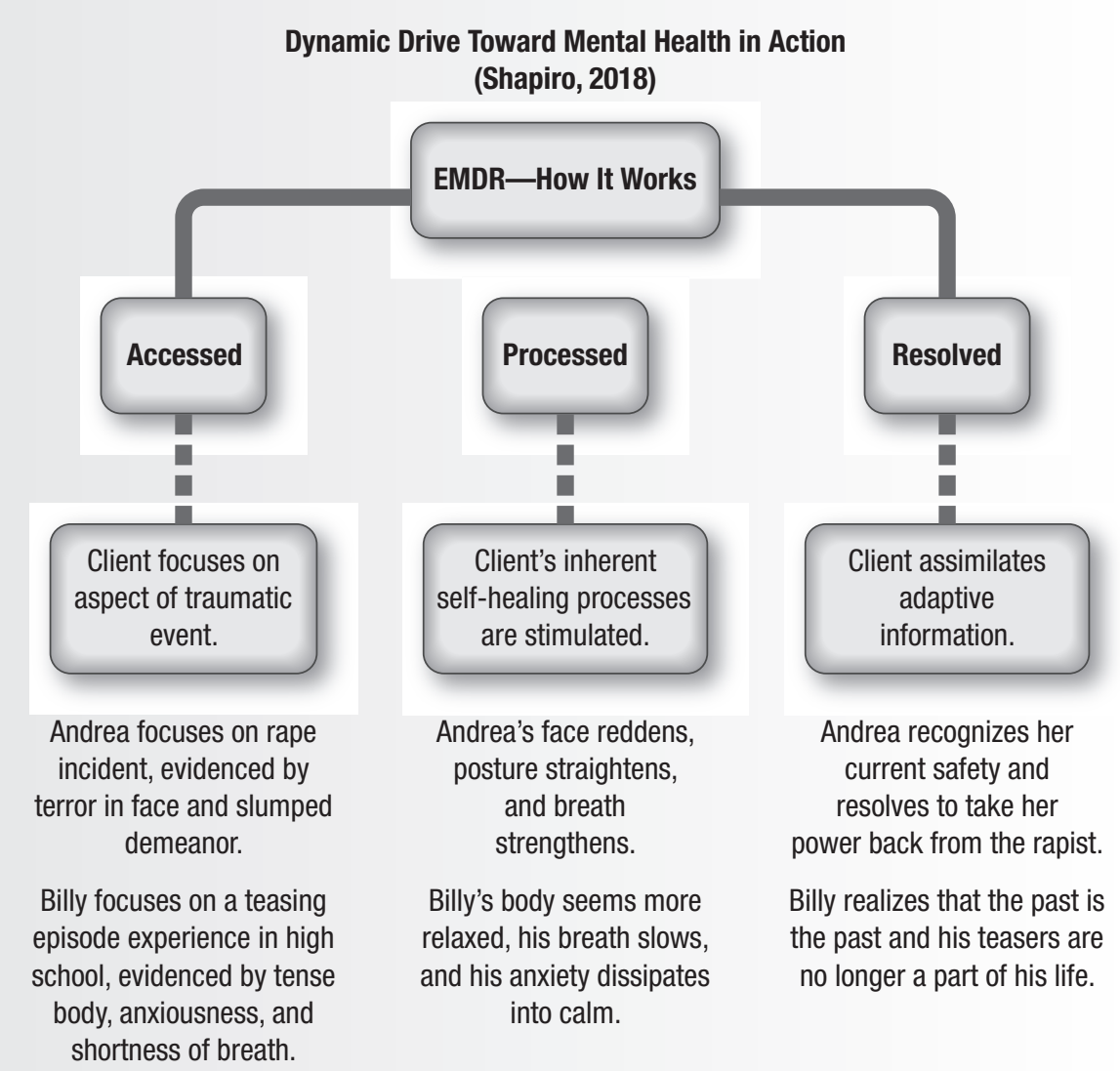

power to terrorize me!" Or consider Billy, who was teased unmercifully in high school by his football teammates. Prior to desensitization and reprocessing, he appeared tense, anxious, and short of breath. After just a few sets of BLS, his body appeared more relaxed, his breath slowed, and his anxiety dissipated into a state of calm. "That was then. This is now. They are nobody to me now."

Exhibit 1.2 demonstrates in action the inherent information processing mechanism as it highlights the changes that occurred as a result of Andrea and Billy's dynamic drive toward mental health with EMDR therapy.

Because the heart of EMDR therapy is the AIP model, it is critical that the clinician has a clear understanding of it before proceeding. An adequate conceptual understanding helps the clinician determine a client's appropriateness for EMDR therapy, as well as explain the process to the client during the Preparation Phase, so that he has some understanding of the 


TABLE 1.7 Client's Experience: Adaptive Information Processing in
Action

Abbreviation: AIP, Adaptive Information Processing.

potential treatment effects. Table 1.7 highlights the before and after treatment changes of EMDR in terms of the AIP model.

Using an example of a traumatic event, Table 1.8 illustrates more graphically what happens during reprocessing of a traumatic event.

For a more comprehensive explanation of AIP, read Shapiro's "Chapter 2, Adaptive Information Processing, the Model as a Working Hypothesis," in Eye Movement Desensitization and Reprocessing: Basic Principles, Protocols, and Procedures (3rd ed.; 2018) and "EMDR, Adaptive Information Processing, and Case Conceptualization" (Shapiro, 2007a).

As the previous tables and figures demonstrate, EMDR is an "empowerment" therapy as it provides space for a client to realize and regain personal power.

\section{MODEL, METHODOLOGY, AND MECHANISM OF EMDR THERAPY}

Why and how does EMDR therapy work? What are the fundamental procedures and elements that contribute to the EMDR therapy overall treatment effect? Unfortunately, no one really knows the neurobiological underpinnings for why EMDR therapy works. Many 
TABLE 1.8 Transmutation of the Targeted Memory (e.g., What Happens During Reprocessing)

NEGATIVE EXPERIENCE ADAPTIVE LEARNING EXPERIENCE

Example: After a heated argument, Mary is raped by her ex-boyfriend in the parking lot of a bar they just left.

$\begin{array}{lll}\text { Mary experiences: } & & \text { Mary experiences: } \\ \text { Intrusive images: } & & \text { Cognitive restructuring of perceptions } \\ \text { Negative thoughts and self-beliefs (e.g., } & & \text { Association to positive affects } \\ \text { I am a bad person) } & \text { Sense of well-being and self-efficacy } \\ \text { Negative emotions (e.g., shame, fear, } & \text { New insights } \\ \text { anger, anxiety) } & \text { Negative physical sensations (e.g., heart } & \text { Feelings of empowerment } \\ \text { palpitations, sweaty palms, difficulty } & \text { Learning } \\ \text { breathing) } & \text { Sense of powerlessness } & \text { Greater sense of understanding } \\ \text { Lack of choice } & & \\ \text { Lack of control } & & \\ \text { Inadequacy } & \end{array}$

questions remain to be answered and, at the same time, clients experience continued positive clinical effects. The following are the three primary aspects of EMDR therapy:

1. Model-The AIP model provides the theoretical model for EMDR therapy.

2. Methodology_Eight phases of EMDR therapy plus the ethics, safeguards, and validated modifications for basic and specific clinical situations and populations.

3. Mechanism-Current hypotheses on how and why EMDR therapy works on a neurobiological level.

\section{MODEL-HOW?}

The AIP model guides its clinical practice (i.e., case conceptualization and treatment planning) and predicts the EMDR treatment effects, and it is independent from the "why" stated later. It is through the lens of the AIP model that the developmental phenomenon is understood, any clinical phenomena that arise during EMDR processing are interpreted, and successful application and positive treatment outcomes are predicted.

Guided by this information processing model, memory networks are believed to form the basis of clinical symptoms and mental health in general, and "unprocessed memories are considered to be the primary basis of pathology" (Shapiro, 2009-2017a). The important components of the model as outlined by Shapiro (1995, 2001, 2018, 2009-2017a, 2009-2017b) are summarized in Table 1.9.

There is a body of literature, including both research and case reports on a variety of clinical complaints, that illustrates the predictive value of the AIP in terms of body dysmorphic disorder (Brown et al., 1997); generalized anxiety disorder (Gauvreau \& Bouchard, 2008); olfactory reference syndrome (McGoldrick et al., 2008); panic disorder with agoraphobia (Fernandez \& Faretta, 2007); bonding failures (Madrid et al., 2006); depression (Uribe \& 


\section{TABLE 1.9 Important Components of the Information Processing} Model

The AIP model views maladaptive/negative memory networks as the "underlying basis of both pathology and mental health.”

Disturbing memories are dysfunctionally stored (i.e., perceived in the same way as when the memory was originally formed) and may disrupt the information processing system.

Emotions, physical sensations, and thoughts and beliefs associated with unprocessed memories are experienced by the client as their perceptions in the present link to the historical memory networks.

In order to be interpreted, a client's perceptions of resent situations link into networks of physically stored memories (negative or dysfunctional) from the past. In other words, the past is present.

As the processing begins, disruptions may be caused by high levels of emotional disturbance or dissociation, which can block adaptive processing.

When a client is processing the memory of a traumatic event, they have the opportunity to forge adaptive associations with memory networks of functional information stored in the brain. This EMDR associative process allows these connections to be made.

During processing, the unprocessed elements of a client's memory (i.e., image, thoughts, sounds, emotional and physical sensations, beliefs) have the ability to transform/ transmute to an adaptive resolution. At this point, learning may take place. By discarding maladaptive information and storing adaptive information, a client has new learning that may better inform future experiences and choices.

Abbreviation: AIP, Adaptive Information Processing.

Ramirez,2006); adolescent depression(Bae etal.,2008);PTSD (Moletal., 2005; Rabonietal., 2006); the role of EMDR therapy in medicine (Shapiro, 2014); dental phobia (Doering et al., 2013); perpetrators with a trauma history (Riccietal.,2006); adult attachment (Wesselman \& Potter, 2009); psychosis (Heins et al., 2011; van den Berg \& van den Gaag, 2012; Varese et al., 2012); peer verbal abuse (Teicher et al., 2010); obsessive-compulsive disorder (Nazari et al., 2011); borderline personality disorder (Brown \& Shapiro, 2006); phantom limb pain (de Roos et al., 2010; Russell, 2008; Schneider et al., 2007; Schneider et al., 2008; Wilensky, 2006); physical punishment and mental disorders (Afifi et al., 2012); childhood trauma and children's emerging symptoms (Arseneault et al., 2012); childhood trauma and household dysfunction (Felitti et al., 1998); and biological sensitivity (Obradovic et al., 2010).

\section{METHODOLOGY-HOW/WHAT?}

EMDR therapy employs an eight-phase integrative treatment approach. Often customized to reflect a client's clinical diagnosis or individual presentation, EMDR therapy utilizes a distinct set of procedures and protocols to address a client's presenting issues. BLS is only one component of the methodology that guides this therapeutic practice of EMDR. EMDR 
therapy is also a three-pronged approach that includes a client's past experiences, present triggers, and a future template of how the client may want to be or respond in a situation. EMDR therapy is flexible in that it also combines aspects of a clinician's previous orientations to psychotherapy as part of the process.

For further reading on the "how" and "what" of EMDR therapy, an overview of the model and procedures is elaborated on in Table 1.10.

TABLE 1.10 Model-Adaptive Information Processing and EMDR Therapy Procedures: How/What

\section{7}

Brown, K. W., McGoldrick, T., \& Buchanan, R. (1997). Body dysmorphic disorder: Seven cases treated with eye movement desensitization and reprocessing. Behavioural and Cognitive Psychotherapy, 25(2), 203-207. https://doi.org/10.1017/S1352465800018403

\section{8}

Felitti, V. J., Anda, R. F., Nordenberg, D., Williamson, D. F., Spitz, A. M., Edwards, V., Koss, M. P., \& Marks, J. S. (1998). Relationship of childhood abuse and household dysfunction to many of the leading causes of death in adults: The adverse childhood experiences (ACE) study. American Journal of Preventive Medicine, 14, 245-258. https://doi.org/10.1016/ S0749-3797(98)00017-8

\section{1}

Ray, A. L., \& Zbik, A. (2001). Cognitive behavioral therapies and beyond. In C. D. Tollison, J. R. Satterhwaite, \& J. W. Tollison (Eds.). Practical pain management (3rd ed., pp. 189-208). Lippincott.

Shapiro, F. (2001). Eye movement desensitization and reprocessing: Basic principles, protocols, and procedures (2nd ed.). Guilford Press.

\section{2}

Perkins, B., \& Rouanzoin, C. (2002). A critical evaluation of current views regarding eye movement desensitization and reprocessing (EMDR): Clarifying points of confusion. Journal of Clinical Psychology, 58(1), 77-97. https://doi.org/10.1002/jclp.1130

Shapiro, F. (2002). EMDR as an integrative psychotherapy approach: Experts of diverse orientations explore the paradigm prism. American Psychological Association Press.

\section{4}

Heim, C., Plotsky, P. M., \& Nemeroff, C. B. (2004). Importance of studying the contributions of early adverse experience to neurobiological findings in depression. Neuropsychopharmacology, 29, 641-648. https://doi.org/10.1038/sj.npp.1300397

\section{5}

Gold, S. D., Marx, B. P., Soler-Baillo, J. M., \& Sloan, D. M. (2005). Is life stress more traumatic than traumatic stress? Journal of Anxiety Disorders, 19, 687-698. https://doi. org/10.1016/j.janxdis.2004.06.002

Mol, S. S. L., Arntz, A, Metsemakers, J. F. M, Dinant, G., Vilters-Van Montfort, P. A. P., \& Knottnerus, A. (2005). Symptoms of post-traumatic stress disorder after non-traumatic events: Evidence from an open population study. British Journal of Psychiatry, 186, 494-499. https://doi.org/10.1192/bjp.186.6.494 


\section{TABLE 1.10 Model-Adaptive Information Processing and EMDR} Therapy Procedures: How/What (continued)

2006

Brown, S., \& Shapiro, F. (2006). EMDR in the treatment of borderline personality disorder. Clinical Case Studies, 5(5), 403-420. https://doi.org/10.1177/1534650104271773

Madrid, A., Skolek, S., \& Shapiro, F. (2006). Repairing failures in bonding through EMDR. Clinical Case Studies, 5(4), 271-286. https://doi.org/10.1177/1534650104267403

Raboni, M. R., Tufik, S., \& Suchecki, D. (2006). Treatment of PTSD by eye movement desensitization reprocessing (EMDR) improves sleep quality, quality of life, and perception of stress. Annals of the New York Academy of Sciences, 1071(1), 508-513. https://doi.org/10.1196/annals.1364.054

Ricci, R. J., Clayton, C. A., \& Shapiro, F. (2006). Some effects of EMDR on previously abused child molesters: Theoretical reviews and preliminary findings. Journal of Forensic Psychiatry and Psychology, 17(4), 538-562. https://doi.org/10.1080/14789940601070431

Shapiro, F. (2006). New notes on adaptive information processing: Case formulation principles, scripts, and worksheets. EMDR Humanitarian Assistance Programs.

Uribe, M. E. R., \& Ramirez, E. O. L. (2006). The effect of EMDR therapy on the negative information processing on patients who suffer depression. Revista Electrónica de Motivación y Emoción, 9, 23-24.

Wilensky, M. (2006). Eye movement desensitization and reprocessing (EMDR) as a treatment for phantom limb pain. Journal of Brief Therapy, 5(1), 31-44.

\section{7}

Fernandez, I., \& Faretta, E. (2007). Eye movement desensitization and reprocessing in the treatment of panic disorder with agoraphobia. Clinical Case Studies, 6(1), 44-63. https:// doi.org/10.1177/1534650105277220

Schneider, J., Hofmann, A., Rost, C., \& Shapiro, F. (2007). EMDR and phantom limb pain: Theoretical implications, case study, and treatment guidelines. Journal of EMDR Practice and Research, 1(1), 31-45. https://doi.org/10.1891/1933- 3196.1.1.31

Shapiro, F. (2007a). EMDR, adaptive information processing, and case conceptualization. Journal of EMDR Practice and Research, 1(2), 68-87. https://doi.org/10.1891/19333196.1.2.68

Shapiro, F. (2007b). EMDR and case conceptualization from an adaptive information processing perspective. In F. Shapiro, F. Kaslow, \& L. Maxfield (Eds.), Handbook of EMDR and family therapy processes (pp. 3-36). John Wiley \& Sons.

Shapiro, F., Kaslow, F. W., \& Maxfield, M. (2007). Handbook of EMDR and family therapy processes. John Wiley \& Sons.

\section{8}

Bae, H., Kim, D., \& Park, Y. C. (2008). Eye movement desensitization and reprocessing for adolescent depression. Psychiatry Investigation, 5(1), 60-65. https://doi.org/10.4306/ pi.2008.5.1.60

Gauvreau, P., \& Bouchard, S. (2008). Preliminary evidence for the efficacy of EMDR in treating generalized anxiety disorder. Journal of EMDR Practice and Research, 2(1), 26-40. https://doi.org/10.1891/1933-3196.2.1.26

McGoldrick, T., Begum, M., \& Brown, K. W. (2008). EMDR and olfactory reference syndrome: A case series. Journal of EMDR Practice and Research, 2(1), 63-68. https://doi. org/10.1891/1933-3196.2.1.63 
TABLE 1.10 Model-Adaptive Information Processing and EMDR Therapy Procedures: How/What (continued)

\section{8}

Russell, M. C. (2008). Treating traumatic amputation-related phantom limb pain: A case study utilizing eye movement desensitization and reprocessing within the Armed Services. Clinical Case Studies, 7(2), 136-153. https://doi. org/10.1177/1534650107306292

Schneider, J., Hofmann, A., Rost, C., \& Shapiro, F. (2008). EMDR in the treatment of chronic phantom limb pain. Pain Medicine, 9(1), 76-82. https://doi.org/10.1111/j.15264637.2007.00299.x

Solomon, R. M., \& Shapiro, F. (2008). EMDR and the adaptive information processing model-Potential mechanisms of change. Journal of EMDR Practice and Research, 2(4), 315-325. https://doi.org/10.1891/1933-3196.2.4.315

\section{9}

Wesselmann, D., \& Potter, A. E. (2009). Change in adult attachment status following treatment with EMDR: Three case studies. Journal of EMDR Practice and Research, 3(3), 178-191. https://doi.org/10.1891/1933-3196.3.3.178

\section{0}

de Roos, C., Veenstra, A., de Jongh, A., den Hollander-Gijsman, M., van der Wee, N., Zitman, F., \& van Rood, Y. R. (2010). Treatment of chronic phantom limb pain using a trauma-focused psychological approach. Pain Research \& Management, 15(2), 65-71. https://doi.org/10.1155/2010/981634

Obradovic, J., Bush, N. R., Stamperdahl, J., Adler, N. E., \& Boyce, W. T. (2010). Biological sensitivity to context: The interactive effects of stress reactivity and family adversity on socioemotional behavior and school readiness. Child Development, 1, 270-289. https://doi. $\operatorname{org} / 10.1111 / j .1467-8624.2009 .01394 . x$

Robinson, J. S., \& Larson, C. (2010). Are traumatic events necessary to elicit symptoms of posttraumatic stress? Psychological Trauma: Theory, Research, Practice, and Policy, 2, 71-76. https://doi.org/10.1037/a0018954

Teicher, M. H., Samson, J. A., Sheu, Y.-S., Polcari, A., \& McGreenery, C. E. (2010). Hurtful words: Association of exposure to peer verbal abuse with elevated psychiatric symptom scores and corpus callosum abnormalities. American Journal of Psychiatry, 167, 14641471. https://doi.org/10.1176/appi.ajp.2010.10010030

\section{1}

Arseneault, L., Cannon, M., Fisher, H. L., Polanczyk, G., Moffitt, T. E., \& Caspi, A. (2011). Childhood trauma and children's emerging psychotic symptoms: A genetically sensitive longitudinal cohort study. American Journal of Psychiatry, 168, 65-72. https://doi. org/10.1176/appi.ajp.2010.10040567

Heins, M., Simons, C., Lataste, T., Pfeifer, S., Versmissen, D., Lardinois, M., Marcelis, M., Delespaul, P., Krabbendam, L., van Os, J., \& Myin-Germeys, I. (2011). Childhood trauma and psychosis: A case-control and case-sibling comparison across different levels of genetic liability, psychopathology, and type of trauma. American Journal of Psychiatry, 168, 1286-1294. https://doi.org/10.1176/appi.ajp.2011.10101531 


\section{TABLE 1.10 Model-Adaptive Information Processing and EMDR} Therapy Procedures: How/What (continued)

2011

Nazari, H., Momeni, N., Jariani, M., \& Tarrahi, M. J. (2011). Comparison of eye movement desensitization and reprocessing with citalopram in treatment of obsessive-compulsive disorder. International Journal of Psychiatry in Clinical Practice, 15(4), 270-274. https:// doi.org/10.3109/13651501.2011.590210

\section{2}

Afifi, T. O., Mota, N. P., Dasiewicz, P., MacMillan, H. L., \& Sareen, J. (2012). Physical punishment and mental disorders: Results from a nationally representative US sample. Pediatrics, 130, 184-192. https://doi.org/10.1542/peds.2011-2947

Shapiro, F. (2012a). EMDR therapy: An overview of current and future research. European Review of Applied Psychology, 62(4), 193-195. https://doi.org/10.1016/j.erap.2012.09.005 van den Berg, D. P. G., \& van der Gaag, M. (2012). Treating trauma in psychosis with EMDR: A pilot study. Journal of Behavior Therapy and Experimental Psychiatry, 43(1), 664-671. https://doi.org/10.1016/j.jbtep.2011.09.011

Varese, F., Smeets, F., Drukker, M., Lieverse, R, Lataster, T., Viechtbauer, W., Read, J., van Os, J., \& Bentall, R. P. (2012). Childhood adversities increase the risk of psychosis: A metaanalysis of patient-control, prospective- and cross-sectional cohort studies. Schizophrenia Bulletin, 38(4), 661-671. https://doi.org/10.1093/schbul/sbs050

\section{3}

Doering, S., Ohlmeier, M.-C., de Jongh, A., Hofmann, A., \& Bisping, V. (2013). Efficacy of a trauma-focused treatment approach for dental phobia: A randomized clinical trial. European Journal of Oral Sciences, 121(6), 584-593. https://doi.org/10.1111/eos.12090

Faretta, E. (2013). EMDR and cognitive behavioral therapy in the treatment of panic disorder: A comparison. Journal of EMDR Practice and Research, 7, 121-133. https://doi. org/10.1891/1933-3196.7.3.121

\section{4}

Read, J., Fosse, R., Moskowitz, A., \& Perry, B. (2014). The traumagenic neurodevelopmental model of psychosis revisited. Neuropsychiatry, 4(1), 65-79. https://doi.org/10.2217/ NPY.13.89

Shapiro, F. (2014). The role of eye movement desensitization and reprocessing (EMDR) therapy in medicine: Addressing the psychological and physical symptoms stemming from adverse life experiences. The Permanente Journal, 18, 71-77. https://doi.org/10.7812/ TPP/13-098

\section{5}

Allon, M. (2015). EMDR group therapy with women who were sexually assaulted in the Congo. Journal of EMDR Practice and Research, 9, 28-34. https://doi.org/10.1891/19333196.9.1.28

Behnam Moghadam, M., Alamdari, A. K., Behnam Moghadam, A., \& Darban, F. (2015). Effect of EMDR on depression in patients with myocardial infarction. Global Journal of Health Science, 7, 258-262. https://doi.org/10.5539/gjhs.v7n6p258 
TABLE 1.10 Model-Adaptive Information Processing and EMDR Therapy Procedures: How/What (continued)

\section{8}

Shapiro, F. (2018). Eye movement desensitization and reprocessing: Basic principles, protocols and procedure (3rd ed.). Guilford Press.

Gauhar, M., \& Wajid, Y. (2016). The efficacy of EMDR in the treatment of depression. Journal of EMDR Practice and Research, 10(2), 59-69. https://doi.org/10.1891/19333196.10.2.59

\section{MECHANISM-WHY?}

Over the past 25 years, numerous studies have indicated that eye movements have effects on a client's memory in terms of vividness, retrieval, emotional arousal, and more. Studies that evaluated EMDR therapy's mechanism of action are listed in Table 1.11.

\section{TABLE 1.11 Mechanism of Action-Why}

\section{6}

Andrade, J., Kavanagh, D., \& Baddeley, A. (1997). Eye-movements and visual imagery: A working memory approach to the treatment of post-traumatic stress disorder. British Journal of Clinical Psychology, 36, 209-223. https://doi.org/10.1111/j.2044-8260.1997. tb01408.x

Armstrong, M. S., \& Vaughan, K. (1996). An orienting response model of eye movement desensitization. Journal of Behavior Therapy \& Experimental Psychiatry, 27, 21-32. https:// doi.org/10.1016/0005-7916(95)00056-9

MacCulloch, M. J., \& Feldman, P. (1996). Eye movement desensitization treatment utilizes the positive visceral element of the investigatory reflex to inhibit the memories of posttraumatic stress disorder: A theoretical analysis. British Journal of Psychiatry, 169(5), 571-579. https://doi.org/10.1192/bjp.169.5.571

Wilson, D., Silver, S. M., Covi, W., \& Foster, S. (1996). Eye movement desensitization and reprocessing: Effectiveness and autonomic correlates. Journal of Behaviour Therapy and Experimental Psychiatry, 27, 219-229. https://doi.org/10.1016/S00057916(96)00026-2

\section{9}

Lipke, H. (1999). Comments on "thirty years of behavior therapy" and the promise of the application of scientific principles. The Behavior Therapist, 22, 11-14.

Rogers, S., Silver, S., Goss, J., Obenchain, J., Willis, A., \& Whitney, R. (1999). A single session, controlled group study of flooding and eye movement desensitization and reprocessing in treating posttraumatic stress disorder among Vietnam war veterans: Preliminary data. Journal of Anxiety Disorders, 13, 119-130.

\section{0}

Bergmann, U. (2000). Further thoughts on the neurobiology of EMDR: The role of the cerebellum in accelerated information processing. Traumatology, 6(3), 175-200. https:// doi.org/10.1177/153476560000600303 


\section{TABLE 1.11 Mechanism of Action-Why (continued)}

\section{1}

Kavanagh, D. J., Freese, S., Andrade, J., \& May, J. (2001). Effects of visuospatial tasks on desensitization to emotive memories. British Journal of Clinical Psychology, 40, 267-280. https://doi.org/10.1348/014466501163689

\section{2}

Rogers, S., \& Silver, S. M. (2002). Is EMDR an exposure therapy? A review of trauma protocols. Journal of Clinical Psychology, 58, 43-59. https://doi.org/10.1002/jclp.1128

Stickgold, R. (2002). EMDR: A putative neurobiological mechanism of action. Journal of Clinical Psychology, 58, 61-75. https://doi.org/10.1002/jclp.1129

\section{4}

Barrowcliff, A. L., Gray, N. S., Freeman, T. C. A., \& MacCulloch, M. J. (2004). Eye movements reduce the vividness, emotional valence and electrodermal arousal associated with negative autobiographical memories. Journal of Forensic Psychiatry and Psychology, 15(2), 325-345. https://doi.org/10.1080/14789940410001673042

Suzuki, A., Josselyn, S. A., Frankland, P. W., Masushige, S., Silva, A. J., \& Satoshi, K. (2004). Memory reconsolidation and extinction have distinct temporal and biochemical signatures. Journal of Neuroscience, 24(20), 4787-4795. https://doi.org/10.1523/ jneurosci.5491-03.2004

\section{6}

Lee, C. W., Taylor, G., \& Drummond, P. D. (2006). The active ingredient in EMDR: Is it traditional exposure or dual focus of attention? Clinical Psychology and Psychotherapy, 13, 97-107. https://doi.org/10.1002/cpp.479

Servan-Schreiber, D., Schooler, J., Dew, M. A., Carter, C., \& Bartone, P. (2006). EMDR for PTSD: A pilot blinded, randomized study of stimulation type. Psychotherapy and Psychosomatics, 75, 290-297. https://doi.org/10.1159/000093950

\section{7}

Propper, R., Pierce, J. P., Geisler, M. W., Christman, S. D., \& Bellorado, N. (2007). Effect of bilateral eye movements on frontal interhemispheric gamma EEG coherence: Implications for EMDR therapy. Journal of Nervous and Mental Disease, 195, 785-788. https://doi. org/10.1097/NMD.0b013e318142cf73

\section{8}

Bergmann, U. (2008). The neurobiology of EMDR: Exploring the thalamus and neural integration. Journal of EMDR Practice and Research, 2(4), 300-314. https://doi. org/10.1891/1933-3196.2.4.300

Sack, M., Hofmann, A., Wizelman, L., \& Lempa, W. (2008). Psychophysiological changes during EMDR and treatment outcome. Journal of EMDR Practice and Research, 2, 239-246. https://doi.org/10.1891/1933-3196.2.4.239

Sack, M., Lempa, W., Steinmetz, A., Lamprecht, F., \& Hofmann, A. (2008). Alterations in autonomic tone during trauma exposure using eye movement desensitization and reprocessing (EMDR) - Results of a preliminary investigation. Journal of Anxiety Disorders, 22, 1264-1271. https://doi.org/10.1016/j.janxdis.2008.01.007

Elofsson, U. O. E., von Scheele, B., Theorell, T., \& Sondergaard, H. P. (2008). Physiological correlates of eye movement desensitization and reprocessing. Journal of Anxiety Disorders, 22, 622-634. https://doi.org/10.1016/ j.janxdis.2007.05.012 


\section{TABLE 1.11 Mechanism of Action-Why (continued)}

\section{8}

Stickgold, R. (2008). Sleep-dependent memory processing and EMDR action. Journal of EMDR Practice and Research, 2, 289-299. https://doi.org/10.1891/1933-3196.2. 4.289

\section{9}

Friedman, D., Goldman, R., Stern, Y., \& Brown, T. (2009). The brain's orienting response: An event-related functional magnetic resonance imaging investigation. Human Brain Mapping, 30(4), 1144-1154.

Gunter, R., \& Bodnar, G. (2009). EMDR works ... But how? Recent progress in the search for treatment mechanisms. Journal of EMDR Practice and Research, 3, 161-168.

Lilley, S. A., Andrade, J., Turpin, G., Sabin-Farrell, R., \& Holmes, E. A. (2009). Visuospatial working memory interference with recollections of trauma. British Journal of clinical psychology, 48(3), 309-321.

\section{0}

Bergmann, U. (2010). EMDR's neurobiological mechanisms of action: A survey of 20 years of searching. Journal of EMDR Research and Practice, 4, 22-42. https://doi. org/10.1891/1933-3196.4.1.22

Hornsveld, H. K., Landwehr, F., Stein, W., Stomp, M., Smeets, S., \& van den Hout, M. A. (2010). Emotionality of loss-related memories is reduced after recall plus eye movements but not after recall plus music or recall only. Journal of EMDR Practice and Research, 4, 106-112. https://doi.org/10.1891/1933-3196.4.3.106

Kapoula, Z., Yang Q., Bonnet, A., Bourtoire, P., \& Sandretto, J. (2010). EMDR effects on pursuit eye movements. PLoS ONE, 5(5), e10762. https://doi.org/10.1371/journal. pone.0010762

van den Hout, M. A., Engelhard, I. M., Smeets, M. A., Hornsveld, H., Hoogeveen, E., \& de Heer, E. (2010). Counting during recall: Taxing of working memory and reduced vividness and emotionality of negative memories. Applied Cognitive Psychology, 24(3), 303-311. https://doi.org/10.1002/acp.1677

\section{1}

El Khoury-Malhame, M., Lanteaume, L., Beetz, E. M., Roques, J., Reynaud, E., Samuelian, J. -C., Blin, O., Garcia, R., \& Khalfa, S. (2011). Attentional bias in post-traumatic stress disorder diminishes after symptom amelioration. Behavior Research and Therapy, 49(11), 796-801. https://doi.org/10.1016/j.brat.2011.08.006

Kristjánsdóttir, K., \& Lee, C. M. (2011). A comparison of visual versus auditory concurrent tasks on reducing the distress and vividness of aversive autobiographical memories. Journal of EMDR Practice and Research, 5, 34-41. https://doi. org/10.1891/1933-3196.5.2.34

\section{2}

van den Hout, M., \& Engelhard, I. (2012). How does EMDR work? Journal of Experimental Psychopathology, 3, 724-738. https://doi.org/10.5127/jep.028212

van den Hout, M. A., Rijkeboer, M. M., Engelhard, I. M., Klugkist, I., Hornsveld, H., Toffolo, M. J., \& Cath, D. C. (2012). Tones inferior to eye movements in the EMDR treatment of PTSD. Behaviour Research and Therapy, 50, 275-279. https://doi. org/10.1016/j.brat.2012.02.001 


\section{TABLE 1.11 Mechanism of Action-Why (continued)}

2013

de Jongh, A., Ernst, R., Marques, L., \& Hornsveld, H. (2013). The impact of eye movements and tones on disturbing memories involving PTSD and other mental disorders. Journal of Behavior Therapy and Experimental Psychiatry, 44(4), 477-483. https://doi.org/10.1016/j. jbtep.2013.07.002

Pagani, M., Hogberg, G., Fernandez, I., \& Siracusano, A. (2013). Correlates of EMDR therapy in functional and structural neuroimaging: A critical summary of recent findings. Journal of EMDR Practice and Research, 7(1), 29-38. https://doi. org/10.1891/1933-3196.7.1.29

\section{4}

Leer, Engelhard, and van den Hout provided corroborating evidence that recall with EM causes 24 -h changes in memory vividness/emotionality.

\section{6}

van Schie, K., Engelhard, I. M., Klugkist, I., \& van den Hout, M. A. (2016). Blurring emotional memories using eye movements: Individual differences and speed of eye movements. European Journal of Psychotraumatology, 7, 29476. https://doi.org/10.3402/ ejpt.v7.29476

van Veen, S. C., Engelhard, I. M., \& van den Hout, M. A. (2016). The effects of eye movements on emotional memories: Using an objective measure of cognitive load. European Journal of Psychotraumatology, 7, 30122. https://doi.org/10.3402/ejpt. v7.30122

\section{8}

Calancie, O. G., Khalid-Khan, S., Booij, L., \& Munoz, D. P. (2018). Eye movement desensitization and reprocessing as a treatment for PTSD: Current neurobiological theories and a new hypothesis. Annals of the New York Academy of Science, 1426(1), 127-145. https://doi.org/10.1111/nyas.13882

Abbreviation: EM, eye movement.

\section{THREE-PRONGED APPROACH}

\section{PAST, PRESENT, FUTURE}

EMDR therapy is a three-pronged treatment approach that focuses on reprocessing of past events, current triggering stimuli, and adaptive rehearsal in future situations (Figure 1.2). This may seem simple, but it is often a concept that escapes many newly trained EMDR therapy clinicians.

Regardless of what you as a participant were taught in the earlier didactic trainings, what you most likely will remember is what was on the instructional sheet that sat on your lap. The first question that you asked the client was, "What old issue or old memory would you like to focus on today?" It is important to note that this question was only used in the training exercises and not in daily clinical practice. Fortunately, this teaching method has changed as the training focus now is to establish a more formalized plan that attempts to identify past events (and a touchstone event, if available), present triggers, and a future template, and to encourage the participant to process in this order. Shapiro (2006) referred to this strategy as the Treatment Planning Guide (TPG). 


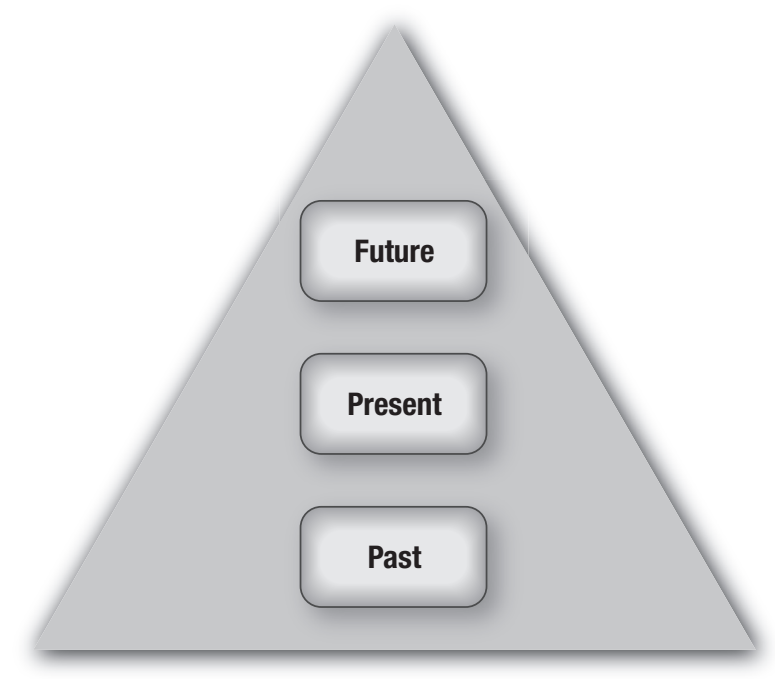

FIGURE 1.2 Self-actualization: EMDR therapy and the three-pronged hierarchy.

To completely resolve a client's issue and achieve adaptive resolution, EMDR therapy is designed to (a) address a client's past events, (b) clean out related current stimuli that might trigger distress in the client and/or reprocess difficult present experiences, and (c) prepare the client for future situations involving the same kind of circumstances (or reaction). The concept of the three-pronged approach is so important that an entire chapter in this Primer has been devoted to it (Chapter 4).

\section{THREE-PRONGED TARGETS-EXPERIENTIAL CONTRIBUTORS TO PRESENT-DAY PROBLEMS}

The order of the processing is important. First, it is necessary to strive to adaptively resolve past traumas, then process current stimuli that trigger distress, and finally do a future template on the present trigger. See Exhibit 1.3 for a breakdown of what is identified and processed under each prong of the EMDR approach.

The clinician may want to consider targeting the memories that lay the groundwork for any present problems and/or issues first. It may be a single traumatic event or what is called a touchstone event, a primary and self-defining event in the client's life. In AIP language, Dr. Shapiro refers to the touchstone memory as a node to which similar events will attach in the continuous formation of a "neuro" or memory network that is critical to the client's sense of self. It is a portal into the memory network as different associations may arise (2018; see Exhibit 1.4A and 1.4B).

Once all presently charged past events are processed (i.e., after the touchstone event is processed), other past events may or may not have a cognitive or affective charge remaining. The clinician may want to consider processing those that have a "charge" before continuing to recent events. Then any recent events, circumstances, situations, stressors, or other triggers that might elicit a disturbance are targeted. After the past events and present disturbances 
EXHIBIT 1.3 Three-Pronged Targets: Order of Reprocessing

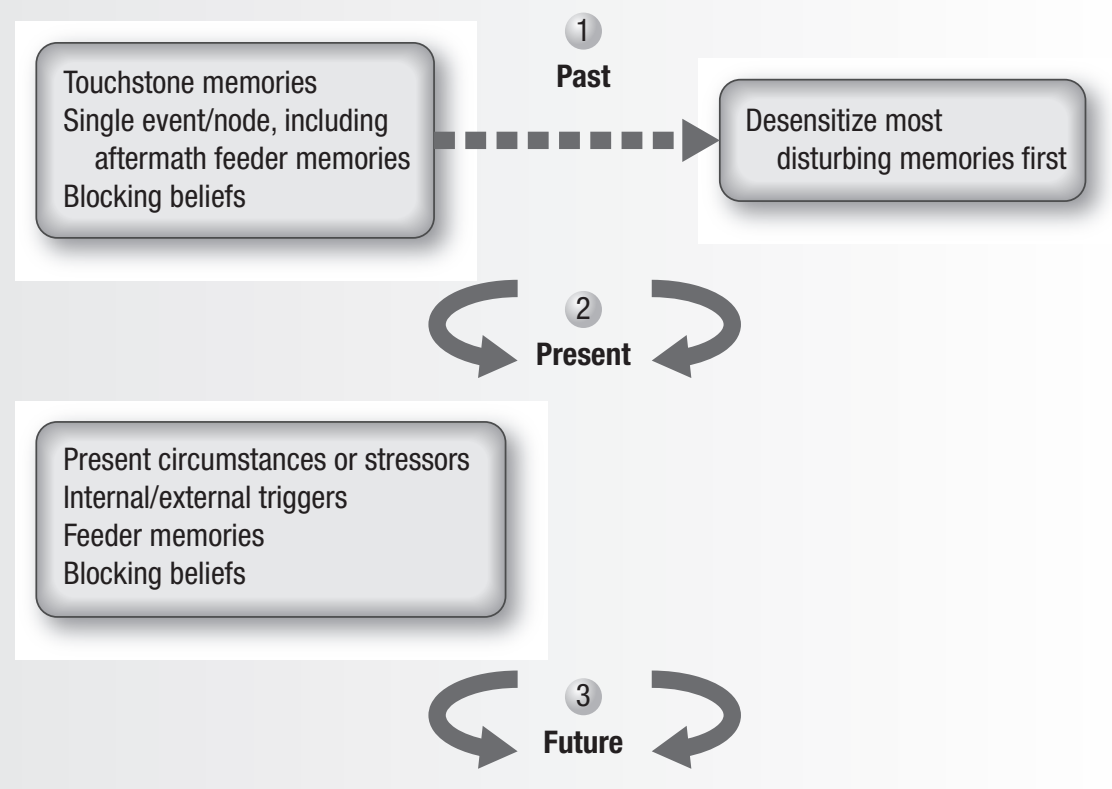

Future desired state (i.e., desired thoughts and actions for the future); incorporating a future or positive template that will accelerate learning new and healthier skills, attitudes, and behaviors

Anticipated anxieties

Development of more adaptive, alternative patterns of response

Feeder memories

Blocking beliefs

have been identified and reprocessed, focus on the future desired behavior and the client's ability to make better choices or cope more effectively. This entails education, modeling, and targeting what Dr. Shapiro calls a future or positive template (2018). It is important for the client to appropriately and properly assimilate the new information gained through the previous prongs (i.e., past, present, and future) by providing them with experiences that ensure future successes.

During recent EMDR trainings, the order of processing the three prongs (i.e., past, present, and future) and strategically identifying the touchstone event, if any, have been emphasized more dramatically. If trained in EMDR prior to 2008, the clinician needs to pay particular attention to Chapters 3 and 4 of this Primer.

Although the standard EMDR therapy protocol prescribes the order of processing as past, present, and future, this is not always practical or effective. Symptoms, circumstances, 
EXHIBIT 1.4 (A) Targets or nodes and (B) targets or nodes with examples.

\section{Targets or Nodes}

(Memories/experiences

to be targeted)
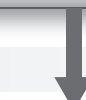

\section{Channels of Associated}

Information

(Dysfunctionally stored information)

(A)

Belief
Participant
Stimuli
Type of event

Physical sensations

Emotional sensations

Single event

Targets or Nodes

(Being bitten by a dog)

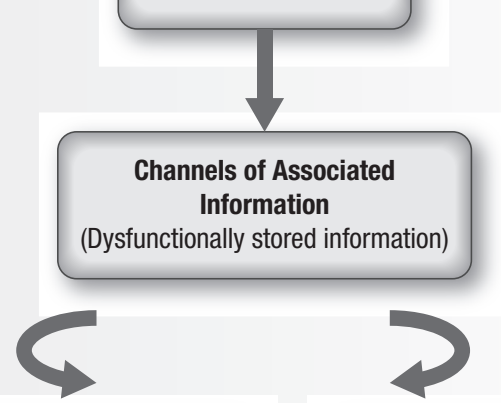

"I am unsafe"

Image of dog's bared teeth

Smell of dog's breath

Sound of dog running down the driveway

(B)

Heart pounding

Can't catch breath

Fear

Frustration

Confusion

Dog bite 
and other variables may indicate future, present, past (e.g., Inverted; Hofmann, 2005 or Reverse; Adler-Tapia, 2015 protocols) or present (e.g., recent events). The client's goals for therapy and presenting issue should determine the order of processing and the clinician should be prepared to direct the client where they need to go for a positive treatment outcome.

\section{THE IMPORTANCE OF PAST, PRESENT, AND FUTURE IN EMDR THERAPY}

The foundation of the three-pronged protocol postulates that earlier memories are processed before current events, and current events are processed before future events. Why is it so important to process these events in this order? What is the effect on the overall treatment result if it is not processed in order of past, present, and future? Earlier life experiences set the groundwork for present events and triggers. Hence, it is useful to reprocess as many of the historical associations with the triggers as possible. Once these associations have been transformed, some, if not many, present triggers will dissipate. There may, however, still be current triggers that exist outside of these channels of association that will need to be targeted and processed independently. Or there may be unprocessed material that surfaces when processing these triggers. These triggers will be the next targets to be processed.

The focus on the future template provides the client an opportunity to imaginally rehearse future circumstances and desired responses. This is yet another opening for unprocessed material to surface. The use of the future template provides the client a means of resolving any anticipatory anxiety that they may still experience in similar future situations. The three-pronged approach appears to be a bottom-up process in that the future is subsumed by the present and the present is subsumed by the past. It has been suggested that bypassing the three-pronged approach as part of the full EMDR treatment means obtaining only a fraction of the full treatment effect. If one does not complete the full protocol and believes that the material is resolved because the past has been successfully reprocessed, the client may remain unprepared for being triggered in the present and may still hold anxieties about the future.

\section{TARGETING POSSIBILITIES}

\section{TARGETS MAY ARISE IN ANY PART OF THE EMDR THERAPY PROCESS}

When a clinician instructs a client to focus on a target in EMDR reprocessing, they are asking the client to tune into a specific memory, image, person, or event or the most disturbing part of it. The target or node then becomes the pivotal point of entry into the associated psychologically stored material. If Raymond's presenting issue relates to the way he responds to his mother-in-law when she first sees him, the target he selects may be the image of her hugging and kissing him as a form of greeting. Because the target image has a constellation of associated experiences around it, Shapiro (2018) calls it a node.

Throughout Dr. Shapiro's clinical books $(2006,2018)$, she refers to several different targets that may arise in certain parts of the process. The past, present, and future targets referred to earlier are the primary focus in the EMDR therapy training. Her text also introduces the reader to other associated words, such as node, channel, cluster, and progression. Exhibit 1.5 attempts to provide a better understanding of the relationship between these types of targets from a more visual perspective. 


\section{EXHIBIT 1.5 Three-Pronged Targets: Types of Targets}

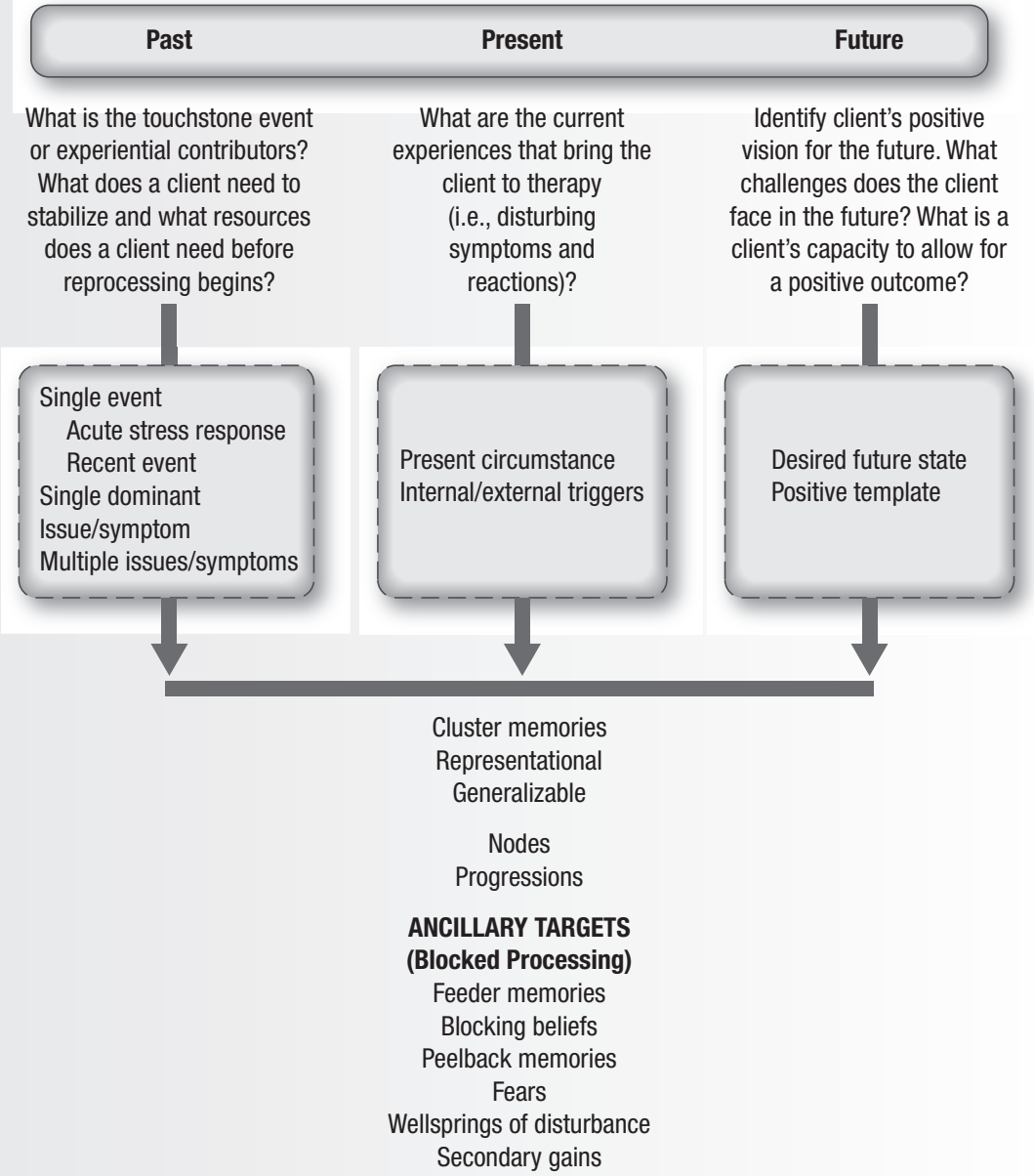

\section{TYPES OF EMDR TARGETS}

As you think about your client sessions, do you recognize any of the types of targets, including the ancillary targets (i.e., other factors that may be contributing to a client's disturbance) listed in Exhibit 1.5? The following definitions are provided as a refresher:

\section{TARGETS FROM THE PAST}

\section{Touchstone Memory}

A memory that lays the foundation for a client's current presenting issue or problem. This is the memory that formed the core of the maladaptive network or dysfunction. It is the first time a client may have believed, "I am not good enough," or that this conclusion was 
formed. The touchstone event often, but not necessarily, occurs in childhood or adolescence. Reprocessing will be more spontaneous for the client if the touchstone events can be identified and reprocessed earlier in the treatment.

Example: As an adult, Mary Jane reported being uncomfortable engaging with large groups of people (i.e., 20 or more). She frequently experienced high levels of anxiety before and during office meetings, in church, and at social events. She was nervous, tentative, fearful, and unsure because she could not trust herself to be in control. During the history-taking process, it was discovered that, when she was in the second grade, Mary Jane wet her pants often. She was afraid to use the restroom because she feared its "tall, dark stalls." Students often teased her, calling her "baby" and yelling out to the other students that she had wet her pants. What she came to believe about herself was, "I cannot trust myself." This belief carried over into her later life and caused her to react tentatively in group situations.

\section{Primary Events}

These are stand-alone events that may emerge during the History-Taking and Treatment Planning, Reprocessing, and Reevaluation Phases, as well as over the course of treatment itself.

Example: Eddie entered therapy months before complaining of headaches and nightmares caused by memories of long-term sexual abuse by his paternal uncle. During treatment, Eddie reported an event involving an automobile accident that occurred 13 years ago. As a result, Eddie still had some anxiousness around driving. This was targeted once the sexual abuse issues had been resolved.

\section{TARGETS FROM THE PRESENT}

\section{Circumstances}

Situations that stimulate a disturbance.

Example: Having his principal observe one of his classes caused Pierre to flush with anxiety, even though he had been Teacher of the Year three times running and was a 25-year veteran in the public school system as a high school teacher.

\section{Internal or External Triggers}

Internal and external cues that can stimulate dysfunctionally stored information and eliciting emotional or behavioral disturbances.

Examples: Sights, sounds, smells, or sensations may be triggers. A client reports becoming triggered by driving on or near a section of roadway where they were involved in a fatal crash in which their best friend was killed. Or a client becomes anxious and ashamed when being questioned by a police officer, even though they have not done anything wrong. The client may react to their own physiological stimuli. For example, they may be triggered by a slightly elevated increase in heart rate, which they fear might lead to a panic attack. One cancer survivor was triggered by an unexplained headache and loss of appetite, leading to fears of recurrence of cancer.

\section{TARGETS FROM THE FUTURE}

\section{Future Desired State}

How would the client like to be feeling, sensing, believing, perceiving, and behaving today and in the future? What changes would be necessary? The third prong of EMDR therapy 
focuses on targeting a positive template that will assist in incorporating positive new learnings and anticipatory events. This stage may involve teaching the client assertiveness skills, modeling good decision making, or having the client imagine future situations, such as coaching people to help them respond more appropriately.

Example: Ryan had always been a passive guy who never could say, "No." "Peace at any cost" was his motto. The touchstone event identified with his conflict-avoidant behavior was a memory of his usually calm mother lunging at his father with a butcher knife during the heat of his father's verbal attack. Before the night was over, his father had beaten his mother so severely that she was hospitalized for three days. Once this memory had been targeted and reprocessed, Ryan felt more empowered but needed instruction on how to stand up for himself more assertively. After assertiveness training, he was able to imagine himself successfully interacting and responding appropriately in conflict-laden circumstances.

\section{Positive Template (i.e., Imaginal Future Template Development)}

A process in which the client uses the adaptive information learned in the previous two prongs to ensure future behavioral success by incorporating patterns of alternative behavioral responses. These patterns require a client to imagine responding differently and positively to real or perceived negative circumstances or situations or significant people.

Example: Joe came home from a business trip and found his wife in bed with his best friend. Joe and his wife had reconciled despite the obvious upheaval it had caused in their already shaky relationship. In the processing of this abrupt discovery, Joe had mostly worked through his reactions and feelings toward his ex-best friend, but he never wanted to interact with him again. However, both worked at the same firm, and it was inevitable that their paths would cross. What the clinician had Joe imagine was a chance meeting with this man and how Joe would like to see this encounter transpire from beginning to end.

\section{OTHER POTENTIAL TARGETS}

\section{Node}

In terms of the AIP model, a node is an associated system of information (Shapiro, 2018). It is "the biologically stored experience central to the memory network designated for therapeutic targeting" (Shapiro, 2009-2017a). It represents a memory network. A node could represent a cluster, a progression, or a feeder memory. To further clarify, a node is the target on which the client focuses during the reprocessing phases; and it represents the unprocessed (i.e., dysfunctional) incident. A target then is the agreed-upon incident the client focuses on and is identified in the initial treatment plan.

Example: Jeremy initially entered therapy because he had difficulty interacting professionally with his supervisor. Whenever his boss called or e-mailed asking him to come to his office, Jeremy felt like a small child being summoned to the principal's office. "What did I do now?" he thought. After a thorough investigation of his past and present, Jeremy related how he felt and reacted around his father. "I always felt as though I had done something wrong." Jeremy's father worked and traveled extensively and was not home very much. When he was, Jeremy could find his father in his office working steadily and mostly unaware of the rest of the family activities in their home. His father was gruff and matter of fact and never paid much attention to Jeremy. When he wanted something from Jeremy or would reprimand him 
for something he did, he would call Jeremy to his office. It was one of those memories that became the target for Jeremy's presenting issue.

\section{Cluster Memories}

These memories form a series of related or similar events and have shared cues, such as an action, person, or location. Each event is representational or generalizable to the other. These nodes are not targeted in the sessions in which they have been identified. The clinician usually keeps an active list of any nodes that arise during reprocessing and reevaluates them later to see if further treatment is necessary.

Example: Anna between the ages of 7 and 10 years was stung by a bee three different times. Each of these events has varying degrees of trauma attached, but each possesses a shared cue, the bees. These are cluster memories and can be grouped together as a single target.

\section{Progression}

A progression is a potential node. It generally arises during the reprocessing of an identified target during or between sets (Shapiro, 2018).

Example: Tricia was targeting incidents related to her mother publicly humiliating her when the memory of how her mother acted at her grandfather's funeral arose. The clinician knew from previous sessions that Tricia had a close, loving relationship with her grandfather and that he was her primary advocate in the family. The clinician wrote down in her notes that her grandfather's funeral may need to be targeted in and of itself. When a progression (i.e., potential target) arises, it is important not to distract the client from her processing of the current target. Rather, the clinician continues to allow the client to follow the natural processing of the present target and note any disturbance around this event that she may need to explore and target during a future session.

\section{Feeder Memory}

This type of memory has been described by Shapiro (2018) as an inaccessible or untapped earlier memory that contributes to a client's current dysfunction and that subsequently blocks its reprocessing. Unlike progressions, which typically arise spontaneously, feeder memories usually are discovered more by direct inquiry and are touchstone memories that are yet to be identified. If a client becomes stuck during reprocessing, there may be a feeder memory stalling the processing. A feeder memory also differs from a progression in that the feeder memory is an untapped memory related to the current memory being processed. When this type of memory emerges during reprocessing, it should be investigated immediately, especially if a client is blocking on an adolescent or adult memory (Shapiro, 2009-2017a, 2009-2017b). A feeder memory is usually treated before the current memory (i.e., EMDR reprocessing within EMDR reprocessing). This is unlike a progression, which is a new target (i.e., memory) that pops up during the processing of another traumatic incident (see earlier, under Progression). The progression is acknowledged and processed later. Direct questioning, floatback, and affect scan may be utilized to identify feeder memories.

Sometimes the identification and spontaneous processing of the feeder memory is sufficient to unblock the processing of the current memory. The feeder memory still needs to be checked following completion of the current memory to determine if it holds any additional 
disturbance. Sometimes, the current memory needs to be contained and the feeder memory reprocessed (i.e., Phases 3-6) before resuming reprocessing of the current memory.

Example: Brittany was in the midst of reprocessing a disturbing event involving malicious accusations by her mother (i.e., "You're a slut." "You must have brought it on somehow." "You deserved everything that happened."). These comments were made by her mother after Brittany at the age of 18 years was nearly raped while walking home from school two months earlier. Following several sets of reprocessing and clinical strategies to unblock or shift her processing, Brittany's level of disturbance did not change. The clinician strategically asked Brittany to focus on the words "I am dirty" (her original negative cognition) and to scan for earlier events in her life that were shameful and humiliating. The memory that finally emerged was the memory of her brothers waving her dirty underwear out a second-story window of their home for all the neighborhood boys to witness. The memory of her brothers' cruel behavior is what is called a feeder memory.

\section{Blocking Belief}

A blocking belief is a belief that stops the processing of an initial target. It is a distorted or irrational conclusion about self, others, or life circumstances (e.g., "It's not safe to get over this problem." "If I feel better, I will forget (betray, dishonor, be disloyal) in the blank)." "I don't deserve to get over this problem."). This type of belief may resolve spontaneously during reprocessing or may require being targeted separately. Blocking beliefs typically show themselves when the clinician is evaluating the Subjective Units of Disturbance (SUD), Validity of Cognition (VoC), or body scan. In the Desensitization Phase, the SUD level will not move below 1; in the Installation Phase, the VoC remains below 7; and in the Body Scan Phase when the body does not clear. Typically, when the clinician asks the client in the Desensitization, Installation, and Body Scan Phases to focus on where the client feels it in their body and, if needed, asks after subsequent sets, "What keeps it a _____?" or "What prevents it (i.e., SUD) from being a 0?" or, if the client is in the Installation Phase, "What keeps it a _____?" or "What prevents it (i.e., VoC) from being a 7 ?" the client may be able to respond with a negative belief and an appropriate, associative early memory. At this point, the processing on the initial target is stopped until the blocking belief memory has been targeted and reprocessed. This does not necessarily require a new target but may be processed with the current target. The direct questioning, floatback, and affect scan techniques are generally useful in identifying blocking beliefs. If after processing there is no change, the clinician may consider the ecological validity of the blocking belief.

Example: Heather, a sergeant in the military, returned home after sustaining injuries during a rocket attack while on a routine field mission in Afghanistan. Two of her fellow soldiers died from the blast. Heather was hit by flying shrapnel that literally left a hole in her leg. She required two subsequent surgeries, neither of which resulted in removing all the rocket shrapnel from her leg. During recuperation, Heather reported disturbing recurring dreams, flashbacks, and thoughts of the rocket attack, which were frequently accompanied by high levels of anxiety or a panic attack. While reprocessing the event, the sergeant's negative cognition was "I'm unsafe" and her PC was "I can be safe." When assessing the sergeant's PC during the Installation Phase, she reported a VoC of 6 . After attempting to shift her response by asking, "Where do you feel it in your body?" with no success, Heather was asked by the clinician, "What prevents it (i.e., VoC) from being a 7 ?" Heather immediately responded with the blocking belief, "I can never be safe." Further questioning by the clinician revealed that, when Heather was 5 years old, she had been digitally penetrated by an older cousin who had said to 
her, "If you tell anyone what happened, you will never be safe. I will find you. And I will kill you." This is also a feeder memory in that it contributes to the current dysfunction and blocked processing. This feeder memory is represented by the blocking belief, "I can never be safe."

\section{Peelback Memory}

A peelback memory usually occurs when a touchstone has not been identified and, during reprocessing, other associations begin to "peel back" to expose prior disturbing memories. There is often confusion between a progression and a peelback memory. A peelback memory is an earlier unsuspected memory, whereas a progression is any new associated memory.

Example: After the processing of an earthquake, Taylor continued to exhibit symptoms of PTSD for which there seemed to be no reason. She continued to have many problems associated with the earthquake even though her house had remained intact, and she and others in her family did not sustain any injuries. Her initial intake showed no indications of previous trauma. On further processing of the earthquake, an early association "peeled back" a memory in her 20s when she was date raped, and then again to an even earlier time when she was molested by a neighbor in her adolescence. Her initial negative cognition, "I am not safe," may have helped to uncover these earlier memories. Unlike a feeder memory, which is an earlier disturbance that blocks the reprocessing of the event, a peelback memory emerges spontaneously during reprocessing. It is similar in terms of the emotional, physical, or cognitive content of the memory being reprocessed, but does not block the processing of the current memory being reprocessed.

\section{Fears}

Fear in the processing of targeted information can become a blocking mechanism. It stalls the process. Dr. Shapiro identified fears to include fear of the clinical outcome of EMDR therapy or the process itself, fear of going crazy, fear of losing good memories, and fear of change. Fear of the process can be readily recognized whenever a client begins to identify elements of EMDR therapy that appear to be problematic for her (2018). Also check to ensure that any expressed fears of the process are not related to secondary gain.

Example: It is not unusual for a client to express concern or fear that they are not "doing it" (i.e., the process) correctly or is afraid of extreme abreaction or that the clinician cannot handle the potential level of distress that they might express during the reprocessing.

\section{Wellsprings of Disturbance}

This phenomenon is indicative of "the presence of a large number of blocked emotions that can be resistant to full EMDR processing" (Shapiro, 2018) and is often caused by the existence of an extensive negative belief system. A wellspring is like a feeder memory in that both feed the emerging emotions. Clients who are resistant to therapy or who seek therapy involuntarily at the urging of someone else (e.g., therapy is court ordered, military command ordered, or requested by a persistent and threatening spouse or parent) are most susceptible to this phenomenon. They are in therapy because of someone else and possess no desire to report or deal with any feelings (Shapiro, 2018).

Example: A man who is forced into therapy at the urging of a disgruntled spouse may possess the belief that "real men don't cry." This belief may be associated with an earlier traumatic memory and result in the client suppressing any high level of disturbance that might 
otherwise naturally occur under a current circumstance (e.g., dealing with his wife's raging episodes). The true level of affective disturbance is never reached by the client, and it is this same level that contributes to the client's present dysfunction. Earlier experiences taught him that men (or boys) are not allowed to express themselves emotionally. If there is no change in the client's imagery, body sensations, or insight, but he continues to report a low level of disturbance, the wellspring phenomenon is probably in effect. When present, the clinician may need to provide additional EMDR strategies to access the blockage. See the formulas in Exhibit 1.6.

EXHIBIT 1.6 Difference Between Wellsprings of Disturbance and Blocking Belief

\section{Blocking Belief $=$ A negative belief about oneself that stalls reprocessing \\ Wellsprings of Disturbance $=$ Negative Beliefs + Unresolved (Early Memories) + Blocked Emotions}

The distinctions between wellsprings of disturbance and blocking beliefs are important because the presence of either determines what course of action a clinician may take to resolve the blocking issues.

\section{Secondary Gain}

A secondary gain issue has the potential of keeping a presenting issue from being resolved.

Example: Typical examples involve the following: what would be lost (e.g., a pension check); what need is being satisfied (e.g., special attention); or how current identity is preserved (e.g., "If I get over my pain, I'm abandoning those who have stood by me since the war." Or, "If I lose my disability, how will I support my family?”).

\section{Channels of Association}

Within the targeted memory, events, thoughts, emotions, and physical sensations may spontaneously arise or arise when a client is instructed to go back to target (i.e., return to the original event [incident, experience]). These are called channels of association and may emerge any time during the reprocessing phases (i.e., Phases 3-6).

When cognition based, channels of association may be another level of the same plateau (responsibility/defectiveness or responsibility/action, safety/vulnerability, or power/control [or choice]) or can be another plateau entirely.

Example: Cara was in the middle of reprocessing being robbed (e.g., safety) after a movie one Friday night. After about five or six sets, she remembered that she had failed to zip up her purse while still in the theater when getting her keys out to drive home. She had a huge bank envelope largely visible for anyone to see (i.e., responsibility). When this channel cleared (i.e., continued to remain positive or remained neutral), the clinician took her back to target, and another channel of association emerged. Cara remembered seeing the robber in the movie theater and that he had been intensely staring at her. She decided it was nothing to worry about and went out to her car (e.g., choice, control). 
Channels of association may emerge during reprocessing, where related memories, thoughts, images, emotions, and sensations are stored and linked to one another. The following example demonstrates a series of channels related to the client's emotions.

Example: Cara was robbed after a movie one Friday night. After one set of BLS, the fear that she reported during the Assessment Phase worsened as her hands began to tremble and chest tightened. When this channel cleared (i.e., continued to remain positive or remained neutral), the clinician took her back to target, and another emotional channel of association emerged. Cara expressed anger with the robber and with herself for being so careless with the bank envelope.

Now that you have a clearer picture of what these targets are and how they are related, can you think of examples for each? Recollect targets from some of your reprocessing sessions with clients to help you identify examples of each. Targets-past, present, future-especially ancillary targets, can emerge in any of the three prongs in the EMDR protocol. Refer to Exhibit 1.7 for assistance. It is important to be on the lookout for them through the entire process to ensure adaptive resolution of every aspect of the client's traumatic history.

\section{BILATERAL STIMULATION}

\section{WHAT DOES IT DO?}

BLS, along with dual attention (i.e., simultaneous awareness of the traumatic memory and the present) and the eight-phase, three-pronged protocol, are the core components of EMDR therapy. Research has validated that BLS makes a unique and effective contribution. The client simultaneously focuses on a negative aspect of an internal experience (e.g., image, thought, emotion, physical sensation) while experiencing rapid, alternating external stimuli (e.g., eye movements, taps, tones).

When Dr. Shapiro was in the early stages of developing the theory, procedures, and protocol behind EMDR therapy, she thought that it was the saccadic eye movements or eye tracking that helped to activate the information processing system, which processes the dysfunctionally stored material around a traumatic event. Alternating bilateral hand taps and auditory tones may also be utilized. The type of BLS utilized is important in terms of what the client can best tolerate while facilitating dual attention. A person with an eye disorder obviously might not be able to track a clinician's fingers well. Someone who does not like to be touched may not be able to tolerate being tapped by the clinician or the proximity of the clinician to them. The type of stimulation chosen depends on the client. Although eye movements are the preferred form of BLS, it is important to be able to offer alternative types of BLS to accommodate the client's needs.

A client's preferred means of BLS is not always the most effective. De Jongh et al.s (2013) discrepant findings "suggest that patients are not the ones that should choose or decide which modality is best for them when they request EMDR therapy." Shapiro (2014) recommends eye movements as the preferred method of BLS, but also suggests that clients be offered tactile or tones when deemed necessary.

If the information during reprocessing is not moving or becomes stuck, it is important to have the client agree beforehand on two preferred directions (i.e., back and forth, up and down, or diagonal) or two types of modalities (i.e., eye movements, audio, tapping) from which the client can choose. Thus, if a need for change in direction or modality occurs, the client has agreed to their preferences in advance. Any time a change in BLS is indicated, the clinician should check with or inform the client that a change is being made before implementing the change. 
EXHIBIT 1.7 Three-Pronged Targets: Types of Targets With Examples

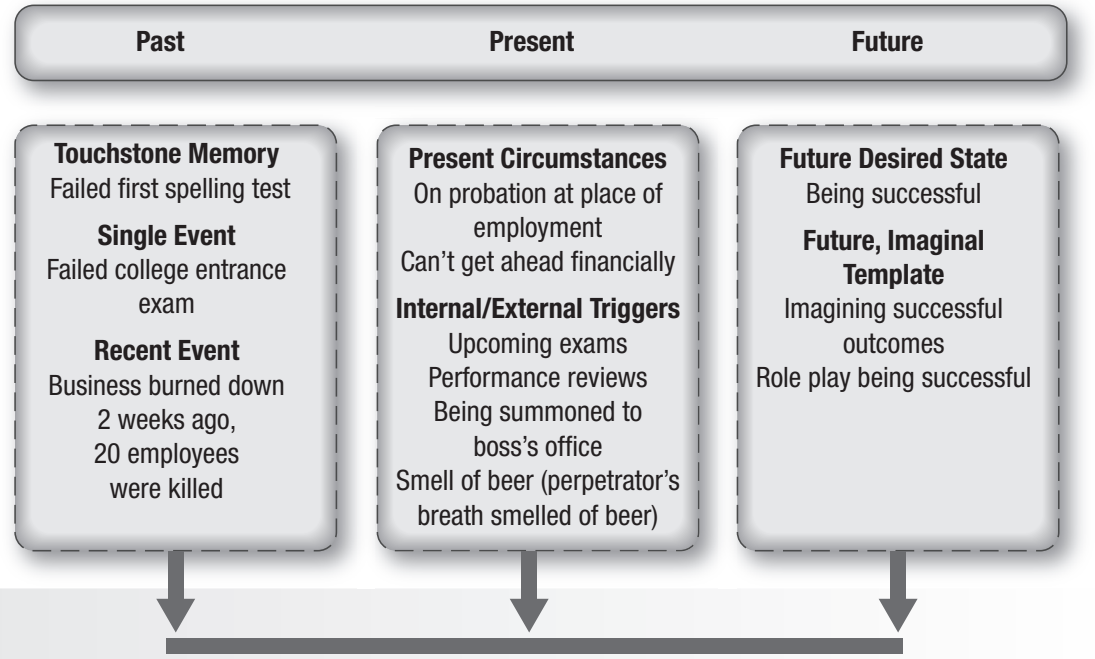

Cluster Memories

Failed driver's license test first time

Flunked gym in 7 th grade

Was asked to quit church choir by music director

\section{Progression \\ (for later processing)}

Was kicked off the football team because he witnessed sexual misconduct between another player and coach

\section{ANCILLARY TARGETS \\ (Block Processing) \\ Feeder Memory \\ I will fail.}

Target earliest memory of having failed

\section{Blocking Belief}

In order to succeed, I need to have my father's approval.

\section{Peelback Memory Rape \\ Fear}

Fear of failure

\section{Wellsprings of Disturbance}

Low level of disturbance; Inability to feel emotions

Target earliest memory of having failed and negative belief that blocks emotions

\section{Secondary Gain}

Fear of loss of identity

Who am I without this fear of failure?

Who am I if I succeed? 


\section{PREFERRED MEANS OF BILATERAL STIMULATION}

Shapiro's (2018) preferred means of BLS is eye movement. All the research involved in establishing the efficacy of EMDR therapy was conducted utilizing eye movements. This type of stimulation also supports dual attention, whereby the client can attend to both internal and external stimuli. The client processes using eye movements with their eyes open so that they remain aware of their present environment.

Many studies have focused on investigating the role of eye movements in EMDR to date (Acierno et al., 1994; Andrade et al., 1997; Barrowcliff et al., 2003, 2004; Boudewyns \& Hyer, 1996; Chemtob et al., 2000; Christman et al., 2003; Christman et al., 2006; Davidson \& Parker, 2001; de Jongh et al., 2013; Devilly et al., 1998; Engelhard et al., 2010a; Engelhard et al., 2010b; Engelhard et al., 2011; Gunter \& Bodner, 2008; Homer et al., 2016; Kavanagh et al., 2001; Kearns and Engelhard, 2015; Kuiken et al., 2002; Kuiken et al., 2010; Lee \& Drummond, 2008; Lohr et al., 1995; Lohr et al., 1996; MacCulloch, 2006; Maxfield et al., 2008; Nieuwenhuis et al., 2013; Parker \& Dagnall, 2007; Parker et al., 2008; Parker et al., 2009; Patel \& McDowall, 2016; Pitman et al., 1996; Propper \& Christman, 2008; Renfrey \& Spates, 1994; Rimini et al., 2016; Sack et al., 2016; Samara et al., 2011; Sanderson \& Carpenter, 1992; Schubert et al., 2011; Servan-Schreiber et al., 2006; Sharpley et al., 1996; Smeets et al., 2012; Solomon et al., 1992; van den Hout et al., 2001; van den Hout et al., 2011, 2012; van Etten \& Taylor, 1998; van Veen et al., 2015; Wilson et al., 1996; Zarghi, 2015).

Two dominant theories have emerged as a result of these research studies about the effects of eye movement during EMDR: (a) eye movements have a tendency to interfere with working memory by reducing emotionality and vividness of autobiographical memories (Andrade et al., 1997; Barrowcliff et al., 2003; Barrowcliff et al., 2004; Engelhard et al., 2010a, 2010b, 2011; Gunter \& Bodner, 2008; Kavanagh et al., 2001; Maxfield et al., 2008; Schubert et al., 2011; van den Hout et al., 2001) and (b) eye movements are linked into the same processes as rapid eye movement (REM) sleep (Barrowcliff et al., 2004; Christman et al., 2003; Christman et al., 2006; Elofsson et al., 2008; Kuiken et al., 2002, 2010; Parker \& Dagnall, 2007; Parker et al., 2008, 2009; Sack et al., 2008; Schubert et al., 2011; Stickgold, 2002, 2008). Stickgold (2002) proposed that "the repetitive redirecting of attention in EMDR induces a neurobiological state, like that of REM sleep" (i.e., the effect of BLS is related to REM in a waking state).

Studies suggest that eye movements are superior to tones (van den Hout et al., 2012) and saccadic eye movements are superior on all parameters in all conditions to vertical eye movements (Parker et al., 2008). One study found that eye movements increased the true memory of an event (Parker et al., 2009). No research presently exists to support having the client process with his eyes closed. Lee and Cuijpers (2013) reported positive effects of eye movements in their meta-analysis.

Table 1.12 outlines the randomized studies of hypotheses regarding eye movements in EMDR reprocessing.

\section{SHORTER OR LONGER? SLOWER OR FASTER?}

During the Preparation Phase, BLS is originally introduced with the safe (calm) place and any other resource enhancement or stabilization exercises deemed appropriate by the clinician prior to using EMDR reprocessing and, then again, during reprocessing in the Desensitization, Installation, and Body Scan Phases. There is a difference in the speed and number of BLS passes used. A single pass in terms of BLS is a "round trip" from center to right, then to left, and back to center again. A series of passes make up a "set." The recommended rate of speed is slower, and the number of round-trip passes is fewer (i.e., 4-6 round 
TABLE 1.12 Hypothesis Regarding Eye Movements-Randomized Studies to Date

1996

Sharpley, C. F., Montgomery, I. M., \& Scalzo, L. (1996). Comparative efficacy of EMDR and alternative procedures in reducing the vividness of mental images. Scandinavian Journal of Behaviour Therapy, 25, 37-42. https://doi. org/10.1080/16506079609456006

EMs superior to control conditions in terms of reducing image vividness.

\section{7}

Andrade, J., Kavanagh, D., \& Baddeley, A. (1997). Eye-movements and visual imagery: A working memory approach to the treatment of posttraumatic stress disorder. British Journal of Clinical Psychology, 36, 209-223. https://doi.org/10.1111/j.2044-8260.1997. tb01408.x

EMs superior to control conditions in terms of reducing image vividness and emotionality.

\section{1}

Kavanagh, D. J., Freese, S., Andrade, J., \& May, J. (2001). Effects of visuospatial tasks on desensitization to emotive memories. British Journal of Clinical Psychology, 40, 267-280. https://doi.org/10.1348/014466501163689

EMs superior to control conditions in terms of reducing within-session image vividness and emotionality.

van den Hout, M., Muris, P., Salemink, E., \& Kindt, M. (2001). Autobiographical memories become less vivid and emotional after eye movements. British Journal of Clinical Psychology, 40(2), 121-130. https://doi.org/10.1348/014466501163535

EMs superior to control conditions in terms of reducing image vividness and emotionality.

\section{2}

Kuiken, D., Bears, M., Miall, D., \& Smith, L. (2002). Eye movement desensitization reprocessing facilitates attentional orienting. Imagination, Cognition and Personality, 21(1), 3-20. https://doi.org/10.2190/L8JX-PGLC-B72R-KD7X

EMs superior to control conditions when correlated with increased attentional flexibility.

\section{3}

Barrowcliff, A., Gray, N., MacCulloch, S., Freeman, T., \& MacCulloch, M. (2003). Horizontal rhythmical eye movements consistently diminish the arousal provoked by auditory stimuli. British Journal of Clinical Psychology, 42(Pt 3), 289-302. https://doi. org/10.1348/01446650360703393

EMs superior to control conditions in terms of reducing arousal provoked by auditory stimuli.

Christman, S. D., Garvey, K. J., Propper, R. E., \& Phaneuf, K. A. (2003). Bilateral eye movements enhance the retrieval of episodic memories. Neuropsychology, 17, 221-229. https://doi.org/10.3758/PBR.15.3.515

Saccadic (not tracking) EMs superior to control condition in episodic retrieval. 
TABLE 1.12 Hypothesis Regarding Eye Movements-Randomized Studies to Date (continued)

2004

Barrowcliff, A. L., Gray, N. S., Freeman, T. C. A., \& MacCulloch, M. J. (2004). Eyemovements reduce the vividness, emotional valence and electrodermal arousal associated with negative autobiographical memories. Journal of Forensic Psychiatry and Psychology, 15(2), 325-345. https://doi.org/10.1080/14789940410001673042

In testing the reassurance reflex model, EMs were found to be superior to control conditions in terms of reducing image vividness and emotionality.

\section{6}

Christman, S. D., Propper, R. E., \& Brown, T. J. (2006). Increased interhemispheric interaction is associated with earlier offset of childhood amnesia. Neuropsychology, 20, 336. https://doi.org/10.1037/0894-4105.20.3.336

EMs may induce a change in: (a) interhemispheric interaction and (b) attendant psychological change in episodic retrieval.

Servan-Schreiber, D., Schooler, J., Dew, M. A., Carter, C., \& Bartone, P. (2006). EMDR for PTSD: A pilot blinded, randomized study of stimulation type. Psychotherapy and Psychosomatics, 75, 290-297. https://doi.org/10.1159/000093950

In this study, patients with single-event PTSD were provided three consecutive sessions, each with a different type of auditory and kinesthetic stimulation; 21 patients with singleevent PTSD (average IES: 49.5) received three consecutive sessions of EMDR with three different types of auditory and kinesthetic stimulation. All three types of stimulation proved useful with the one conferring the additional benefit of alternating stimulation.

\section{7}

Parker, A., \& Dagnall, N. (2007). Effects of bilateral eye movements on gist based false recognition in the DRM paradigm. Brain and Cognition, 63, 221-225. https://doi. org/10.1016/j.bandc.2006.08.005

Bilateral saccadic EM groups "were more likely to recognize previously presented words and less likely to falsely recognize critical non-studied associates" (p. 223).

\section{8}

Gunter, R. W., \& Bodner, G. E. (2008). How eye movements affect unpleasant memories: Support for a working-memory account. Behaviour Research and Therapy, 46(8), 913-931. https://doi.org/10.1016/j.brat.2008.04.006

Support for working-memory account of the benefits of EM.

Lee, C. W., \& Drummond P. D. (2008). Effects of eye movement versus therapist instructions on the processing of distressing memories. Journal of Anxiety Disorders, 22(5), 801-808. https://doi.org/10.1016/j.janxdis.2007.08.007

Significant reduction in distress for EM in post-treatment and follow-up.

Maxfield, L., Melnyk, W. T., \& Hayman, C. A. G. (2008). A working memory explanation for the effects of eye movements in EMDR. Journal of EMDR Practice and Research, 2(4), 247-261. https://doi.org/10.1891/1933-3196.2.4.247

Supported the working memory explanation on the effects of EM on dual-attention tasks on autobiographical memory.

Parker, A., Relph, S., \& Dagnall, N. (2008). Effects of bilateral eye movement on retrieval of item, associative and contextual information. Neuropsychology, 22, 136-145. https://doi. org/10.1037/0894-4105.22.1.136 
TABLE 1.12 Hypothesis Regarding Eye Movements-Randomized Studies to Date (continued)

\section{8}

Bilateral saccadic EM group superior on retrieval of the item and associative and contextual information.

\section{9}

Parker, A., Buckley, S., \& Dagnall, N. (2009). Reduced misinformation effects following saccadic bilateral eye movements. Brain and Cognition, 69, 89-97. https://doi. org/10.1016/j.bandc.2008.05.009

Supports hypothesis of the effects on episodic memory and interhemispheric activation.

\section{0}

Engelhard, I. M., van den Hout, M. A., Janssen, W. C., \& van der Beek, J. (2010a). Eye movements reduce vividness and emotionality of "flashforwards." Behaviour Research and Therapy, 48, 442-447. https://doi.org/10.1016/j.brat.2010.01.003

In non-dual task condition and while thinking of future-oriented images, EMs resulted in reduced ratings in image vividness and emotional intensity.

Engelhard, I. M., van den Hout, M. A., Janssen, W. C., \& van der Beek, J. (2010b). The impact of taxing working memory on negative and positive memories. European Journal of Psychotraumatology, 1, 5623. https://doi.org/10.3402/ejpt.vli0.5623

Compared Tetris to EMs.

Kuiken, D., Chudleigh, M., \& Racher, D. (2010). Bilateral eye movements, attentional flexibility and metaphor comprehension: The substrate of REM dreaming? Dreaming, 20, 227-247. https://doi.org/10.1037/a0020841

Found differential effects between EM and non-EM conditions.

\section{1}

Engelhard, I. M., van den Hout, M. A., Dek, E. C. P., Giele, C. L., van der Wielen, J.-W., Reijnen, M. J., \& van Roij, B. (2011). Reducing vividness and emotional intensity of recurrent "flashforwards" by taxing working memory: An analogue study. Journal of Anxiety Disorders, 25, 599-603. https://doi.org/10.1016/j.janxdis.2011.01.009

Found with EMs on recall only; there was a trend toward reduced vividness of intrusive images and emotionality.

Samara, Z., Bernet M., Elzinga, B. M., Heleen A., Slagter, H. A., \& Nieuwenhuis, S. (2011). Do horizontal saccadic eye movements increase interhemispheric coherence? Investigation of a hypothesized neural mechanism underlying EMDR. Frontiers in Psychiatry, 2, 4. https://doi.org/10.3389/fpsyt.2011.00004

In healthy adults, 30 seconds of bilateral saccadic EMs "enhanced the episodic retrieval of non-traumatic emotional stimuli."

Schubert, S. J., Lee, C. W., \& Drummond, P. D. (2011). The efficacy and psychophysiological correlates of dual-attention tasks in eye movement desensitization and reprocessing (EMDR). Journal of Anxiety Disorders, 25, 1-11. https://doi.org/10.1016/j.janxdis.2010.06.024

EMs led to increased reduction in distress, decrease in heart rate at onset of EMs, decrease in skin conductance during EMs, increase in heart rate variability and respiration as EMs continued, and increased frequency of orienting responses at the start of exposure. van den Hout, M. A., Engelhard, I. M., Rijkeboer, M. M., Koekebakker, J., Hornsveld, H., Leer, A., Toffolo, M. B. J., \& Akse, N. (2011). EMDR: Eye movements superior to beeps in taxing working memory and reducing vividness of recollections. Behaviour Research and Therapy, 49, 92-98. https://doi.org/10.1016/j.brat.2010.11.003 
TABLE 1.12 Hypothesis Regarding Eye Movements-Randomized Studies to Date (continued)

2011

Supports a working memory account of EMDR therapy and that EMs are superior to the effects of beeps on negative memories.

\section{2}

Smeets, M. A. M., Dijs, M. W., Pervan, I., Engelhard, I. M., \& van den Hout, M. A. (2012). Time-course of eye movement-related decrease in vividness and emotionality of unpleasant autobiographical memories. Memory, 20(4), 346-357. https://doi.org/10.1080/0 9658211.2012.665462

Supports the theory that emotionality reduces only after vividness has dropped. van den Hout, M. A., Rijkeboer, M. T., Engelhard, I. M., Klugkist, I., Hornsveld, H., Toffolo, M., \& Cath, D. (2012). Tones inferior to eye movements in the EMDR treatment of PTSD. Behaviour Research and Therapy, 50, 275-279. https://doi.org/10.1016/j. brat.2012.02.001

In this study, EM were proven to be more beneficial than tones. It was unclear if tones added to recall only.

\section{3}

de Jongh, A., Ernst, R., Marques, L, \& Hornsveld, H. (2013). The impact of eye movements and tones on disturbing memories involving PTSD and other mental disorders. Journal of Behavior Therapy and Experimental Psychiatry, 44, 477-483. https://doi.org/10.1016/j. jbtep.2013.07.002

Evidence for the value of employing EMs in EMDR treatment, and evidence that a client's preference of BLS (EMs, tactile, or auditory) may not necessarily be the most effective form of bilateral stimulation.

Nieuwenhuis, S., Elzinga, B. M., Ras, P. H., Berends, F., Duijs, P., Samara, Z., \& Slagter, H. A. (2013). Bilateral saccadic eye movements and tactile stimulation, but not auditory stimulation, enhanced memory retrieval. Brain and Cognition, 81, 52-56. https://doi. org/10.1016/j.bandc.2012.10.003

Functional connectivity between the two hemispheres of the brain increased by bilateral activation of the same.

\section{5}

Kearns, M, Engelhard I. M. (2015). Psychophysiological responsivity to scriptdriven imagery: An exploratory study of the effects of eye movements on public speaking flashforwards. Frontiers in Psychiatry, 6, 115. https://doi.org/10.3389/ fpsyt.2015.00115

Using the control condition of imagery and EM while holding a mental image of a scenario in mind showed a significant decrease in heart rate, thus providing a measure of emotionality.

van Veen, S. C., van Schie, K., Wijngaards-de Meij, L. D., Littel, M., Engelhard, I. M., \& van den Hout, M. A. (2015). Speed matters: Relationship between speed of eye movements and modification of aversive autobiographical memories. Frontiers in psychiatry, 6(45), 1-9. https://doi.org/10.3389/fpsyt.2015.00045

This study demonstrated that image vividness did not decrease emotionality over time. This is consistent with a working memory hypothesis that states that highly vivid images responded better to fast EMs while less vivid ones responded better to slower EMs. 
TABLE 1.12 Hypothesis Regarding Eye Movements-Randomized Studies to Date (continued)

\section{6}

Homer, S. R., Deeprose, C., \& Andrade, J. (2016). Negative mental imagery in public speaking anxiety: Forming cognitive resistance by taxing visuospatial working memory. Journal of Behavior Therapy and Experimental Psychiatry, 50, 77-82. https://doi. org/10.1016/j.jbtep.2015.05.004

It was established as hypothesized that reduction in vividness of represented imagery (i.e., public speaking was visualized less vividly; generated less anxiety when imagined) was more effected by EM than auditory task.

Sack, M., Zehl, S., Otti, A., Lahmann, C., Henningsen, P., Kruse, J., \& Stingl, M. (2016). A comparison of dual attention, eye movements, and exposure only during eye movement desensitization and reprocessing for posttraumatic stress disorder: Results from a randomized clinical trial. Psychotherapy and Psychosomatics, 85(6), 357-365. https://doi. org/10.1159/000447671

When eye fixation and exposure control were compared to bilateral EMs, both were found to be equally effective as well as superior to exposure alone at posttest.

Abbreviations: BLS, bilateral stimulation; EM, eye movement; IES, Impact of Event Scale; PTSD, posttraumatic stress disorder.

trips) when using BLS with resource, coping, relaxation, and stress reduction exercises and strategies. Slower and shorter sets are utilized in stabilization efforts to not activate any disturbing material prior to actual reprocessing.

While using BLS when reprocessing, including installation and, when necessary, the body scan, the speed is tolerably comfortable (i.e., much faster) for the client and number of passes is increased (i.e., from 20 to 24 round trips to start, lengthen to 36 to 40 as needed during the reprocessing phases). The number of sets of BLS is determined by attention to the client's response and is customized to the needs of the client. Faster and longer sets of BLS are more likely to activate linkages to other memory networks and trigger associated channels of information. The longer and faster the eye movement, the faster the associative linkages occur. Always increase the speed of BLS with caution because, if associative linkages come too fast for a client's window of tolerance, it can lead to a loss of dual awareness. Faster BLS may impede effective processing and consequently lead to slower reprocessing. On the other hand, if a client is trying to "think" rather than just observe during reprocessing, faster eye movements can help move him into actual reprocessing.

During the Desensitization Phase, the suggested average number of BLS sets (i.e., 20-24 longer, faster sets, then lengthen to $36-40$ or as needed by the client) is only a starting point for the client's processing. As the clinician wants to avoid sets that are too long or too short, it is suggested that she begin with 20 or more passes and watch the client's facial expressions and body language to determine the best length for any set. After this initial set, the clinician may customize the number of sets according to the client's need or response.

Some clients may require shorter or longer and/or slower or faster sets. For instance, clients who are anxious and/or unsure of the process may require longer sets of BLS. In addition, some clients need more time to shift from an external (outward) to an internal (inward) focus. The longer sets of BLS create adequate time for the client's processing to unfold and increase the likelihood of the client experiencing success with reprocessing. If a clinician watches eyes and other body language, clues will usually arise that indicate the appropriate pace and number of BLS sets. It is always appropriate to ask the client for feedback as well. 


\section{TABLE 1.13 Bilateral Stimulation-Fast or Slow? Short or Long?}

\section{SLOW, SHORT SETS (4-6) \\ ELICIT A RELAXATION RESPONSE (SLOWS DOWN THE TRAIN)}

Safe (calm) place

Resource development

Resource development and installation
FAST, LONG SETS (24-36)

ELICIT AN ACTIVATION RESPONSE (SPEEDS UP THE TRAIN)

In terms of desensitization and speed, eye movements (or passes) that are too slow tend to stimulate a relaxation response and may not facilitate sufficient dual attention. Therefore, it is imperative for optimal processing that the clinician facilitates the passes as fast as the client can comfortably tolerate. In addition, the clinician should avoid long pauses between sets without a specific reason (e.g., the client feels the need for clinician connection). The client is discouraged from talking at any length between sets. It is suggested that the clinician minimally or nonverbally acknowledge what the client has reported and say, "Notice that" or "Go with that."

Although the purpose of the Installation Phase is to fully integrate a positive self-assessment with the targeted information, there is still the possibility that other associations could emerge that may need to be addressed. The faster, longer sets of BLS facilitate the emergence of any lingering disturbing material related to the original targeted event. Remember, a completely successful treatment of the original target memory cannot be attained until the early memories that caused the blocking belief are reprocessed. There is little research regarding this widely practiced distinction in the speed and number of sets of BLS. However, it is considered a guideline by many EMDR trainers, consultants, facilitators, and clinicians.

Except for the reprocessing phases of treatment, shorter, slower sets of BLS (approximately 4-6) are used to reinforce and strengthen the client's positive networks. The clinician wants to ensure that the client's brain is not activated in such a way as to associate the positive networks with maladaptive experiences. Especially with more complex cases, the client may not possess sufficient adaptive memory networks; and the client must have access to adaptive memory networks for reprocessing to occur. During the Installation Phase, the sets of BLS should be longer and faster (i.e., 20-24 to start, lengthen to 36-40 as needed) because the client is strengthening the connection between the newly processed memory and its connection to other existing adaptive information networks and/or continued reprocessing of associated negative networks as well. Therefore, when a clinician wants to manage a client's response, use slow, shorts sets; when a clinician wants to allow a client's associations to roam freely, use longer, faster sets. Table 1.13 demonstrates when to use long or short and slow or fast sets of BLS.

Note: If a positive association occurs during reprocessing, do not slow the speed of the BLS or decrease the number of sets. During Phases 3 to 6, the sets of BLS are faster and longer as there is always the possibility of negative associations emerging at any phase.

\section{CONTINUOUS BILATERAL STIMULATION}

Although there may be clinicians who administer one continuous sequence of BLS throughout the Desensitization, Installation, and Body Scan Phases, Shapiro (2018) clearly outlines the reasons for breaking the stimulation into sets (Table 1.14). 
Note: If a clinician uses continuous BLS during the reprocessing phases 3 through 6 , it should not be called EMDR therapy.

\section{TABLE 1.14 Reasons for Breaking Bilateral Stimulation Into Sets}

Provides an opportunity for the clinician to determine if processing has occurred based upon the client's feedback.

Gives the client a break from processing, especially if a set contained an intense abreactive response.

Helps a client reorient in terms of present time and consequent safety (i.e., helps to keep "one foot in the present") and thereby facilitates dual awareness.

Allows a client to share any new revelations or insights that arise during processing.

Allows a clinician to reaffirm a client's experience throughout the process.

Enables a client to better integrate any new information that emerges on a verbal or conscious level.

Allows a clinician to continually reassess or judge the need for any additional clinical interventions or strategies.

Provides multiple opportunities for a clinician to give and a client to receive encouragement and reassurance.

In terms of abreactive responses, it solidifies for a client that they are larger than the disturbing experience and in control as they demonstrate they can approach and distance from the disturbance during these breaks.

Abbreviation: BLS, bilateral stimulation.

Table 1.15 lists what can happen if BLS (i.e., in terms of speed and number of sets) is used inappropriately.

\section{TABLE 1.15 Derailment Possibilities-Bilateral Stimulation}

Errors

\section{Using long and fast sets when doing stabilization}

Doing so may activate processing prematurely (i.e., speeds up the train) and cause a client to begin processing any dysfunctionally stored past memories or present triggers. Short, slow sets of BLS are indicated when facilitating stabilization.

\section{Using short and slow sets when doing reprocessing}

Slow, short BLS may stimulate a relaxation response and inhibit a client from being appropriately activated by the targeted incident/event. When doing reprocessing, the BLS should be tolerably uncomfortable for the client. Using slow, short sets may also not facilitate dual awareness. It becomes too easy for a client to become absorbed in the memory when following eye movements that are too slow or too short. Long, fast sets of BLS are indicated when reprocessing is occurring.

Note: Wilson et al. (1996) and Schubert et al. (2011) found that faster BLS may at times cause a compelled relaxation response. 


\section{TABLE 1.15 Derailment Possibilities-Bilateral Stimulation}

Errors (continued)

Continuing with eye movements if a client reports pain or dryness because of the process

If this occurs, switch to a previously agreed upon, alternate form of BLS. For example, if a client was reportedly poked in the eye when they were a child, auditory or tactile stimulation may be a better alternative.

\section{Pausing too long in between sets}

There should be a specific reason for pausing too long between sets. Maybe the client is overwhelmed, "needs" to talk, or wants assurance that they are "doing it right." If the client tends to relay everything that happened between sets, gently explain the information they give is for baseline reasons only (i.e., to let the clinician know where they are in the process), that the "mind is faster than the mouth," and only minimal information is needed between sets. When a clinician or client talks excessively between sets, it inhibits the reprocessing (i.e., slows the train) of the targeted material.

\section{During reprocessing, using sets that are too long or too short}

Using sets that are too long may cause a client to lose track of the primary event and become overwhelmed with too many thoughts, images, and emotions, and may inhibit or derail processing. Sets that are too short may inhibit processing by not giving a client enough time to initiate complete processing. The rule of thumb is to start with 20-plus sets of eye movements and judge from a client's response (i.e., facial expression and body language) to determine what the appropriate number of sets is for each individual client. However subtle, a client will usually give a clue as to when a set is complete.

\section{Using continuous BLS (i.e., taking no breaks)}

Using continuous BLS throughout an entire EMDR session follows the same logic previously for long sets. The primary purpose of stopping the train is so dysfunctional information may be unloaded and more adaptive information may be loaded. Also, taking a break between sets helps a client keep "one foot in the present."

\section{Continuing with BLS when the client has indicated that he is finished with the set}

It is not uncommon for a client to look at a clinician and say, "Can we take a break right now? I need to say something." In the event this occurs, simply stop the train and allow the client to unload any material; and restart the train when the client is willing and able.

Abbreviation: BLS, bilateral stimulation.

\section{HOW TO DO EYE MOVEMENTS}

Table 1.16 outlines the specific criteria outlined by Shapiro (2018) on the proper and acceptable way to facilitate eye movements in terms of preference, duration, speed, distance, height, and more. 


\section{TABLE 1.16 How to Do Eye Movements}

\section{Before concentrating on emotionally disturbing material}

Initially using horizontal or diagonal eye movements, find the best fit for a client (e.g., horizontal, vertical, diagonal).

Note: The vertical eye movements may be preferred if a client has a history of vertigo. It is also reported to be helpful in reducing extreme emotional nausea, agitation, eye tension, or dizziness. Vertical eye movements have been known to produce a calming effect.

Experiment to find a client's comfort level (i.e., distancing, speed, height).

Switch immediately to an alternate form of BLS if a client reports eye pain or dryness. Generate a full eye movement set by moving a clinician's hand from one side of a client's range of vision to the other.

The speed of the eye movement should be as rapid as possible and without any undue physical discomfort to a client.

Use at least two fingers as a focal point (i.e., two fingers held together is usually the preferred number in American culture).

With palm up approximately 12 to 14 inches from a client's face (i.e., preferably chin to chin or contralateral eyebrow, some say shoulder to shoulder), hold two fingers upright and ask a client, "Is this comfortable?"

Note: It is important to determine distance and placement of the eye movement with which a client is the most comfortable prior to the Assessment Phase.

Demonstrate the direction of the eye movements by starting in the middle and slowly moving the fingers back and forth in a client's visual field, and then ending in the center.

Evaluate and monitor a client's ability to track the moving fingers. Start slowly and increase the rate of speed to the fastest a client can comfortably tolerate physically and still keep in their window of emotional intensity.

Ask a client during this testing phase if they have any preferences in terms of speed, distance, and height prior to concentrating on any negative emotions or dysfunctional material.

\section{After concentrating on emotionally disturbing material}

Listen to the client's feedback to determine if adequate processing is taking place at the end of a session.

If the client appears comfortable and shifts are occurring, maintain the predetermined speed.

If the client is uncomfortable and shifts are not occurring, the clinician may need to adjust the speed, direction, and number of eye movements.

If the client appears stuck (i.e., after successive sets of eye movements, the client reports no shifts), change the direction of the eye movement.

If a client experiences difficulty (i.e., manifests in irregular eye movements or "bumpiness") following a clinician's fingers, attempt to assist the client to establish a more dynamic connection and sense of movement control that results in smoother tracking by instructing the client to "push my fingers with your eyes."

Assess the client's comfort, preferred speed, and ability to sustain eye movements by listening to their feedback between sets. 


\section{TABLE 1.16 How to Do Eye Movements (continued)}

If the client reports an increase in positive shifts, continue to maintain the same direction, speed, and duration.

Note: If a clinician believes it might be beneficial, it is appropriate to experiment. However, a client's feedback should be the final determinant whether to decrease or increase the duration of a set.

Continue BLS until a shift or plateau is observed in the client, unless the client utilizes their stop signal.

If a client experiences weakness in their eye muscles, they may be unable to do more than a few eye movements at a time.

If a client is experiencing high levels of anxiety, demonstrates a tracking deficit, or finds them aversive, they may be unable to track hand movements.

Note: If this occurs, reprocess the underlying experience of discomfort and, if need be, use the two-handed approach or auditory or tactile stimulation. The two-handed approach entails having the clinician position their closed hands on opposite sides of the client's visual field at eye level and then alternating raising right and left index fingers while instructing the client to move their eyes from one raised finger to the other. This type of BLS creates an orienting or attentional response and eliminates the need for client tracking.

Abbreviation: BLS, bilateral stimulation.

\section{IS BILATERAL STIMULATION EMDR THERAPY?}

BLS is but one component of EMDR therapy. Stimuli, such as directed and accelerated eye movements, are used to activate the client's information processing system as they focus on a past trauma, present-day trigger, or future event. Over the years, many beginning students of EMDR therapy, consultees, and even seasoned veterans have referred to BLS as EMDR. BLS is used when facilitating the sacred space, safe (calm) place, and resource development exercises (Chapter 8). When coupled solely with BLS, does this mean that sacred space, safe (calm) place, resource installation, or even the reprocessing phases are EMDR therapy? EMDR therapy is clearly identified as an eight-phase, three-pronged process. If one of the phases is eliminated or substituted with something else, it can no longer be called EMDR therapy.

\section{IMPORTANT CONCEPTS TO CONSIDER}

\section{MEMORY NETWORK ASSOCIATIONS}

No one knows what a memory network looks like, but these networks represent the basis of the AIP model. Metaphorically, Dr. Shapiro pictures these networks as a series of channels "where related memories, thoughts, images, emotions, and sensations are stored and linked to one another" (Shapiro, 2018). The negative memory network associations may consist of a series of memories linked to a person (e.g., critical mother); auditory, tactile, or other sensory stimuli (e.g., the sound of a car backfiring); events (e.g., automobile accident, plane 
crash, tornado); physical sensations (e.g., chest or leg pain); emotions (e.g., fear, sadness); or beliefs (e.g., I am unlovable). The goal is to assist the client's progression through these memory networks and toward an adaptive resolution.

\section{STOP SIGNAL}

It is important to heed a client's wishes to ensure a sense of safety in the therapeutic environment. EMDR treatment is a choice, and the desire to stop or continue in whatever context is a choice as well. It is one a clinician should always respect. It is imperative a client always feels that the process is a choice and not an imposition or demand. So, if a client asks to stop reprocessing, the clinician should stop immediately.

In the Preparation Phase, the client is asked to provide the clinician with a cue that indicates they want to stop the processing. It may be a hand, finger, or body gesture. The client may simply just turn away or hold their arms up in the air. Whatever the cue is, the processing should be discontinued immediately when used. This allows the client to maintain an ongoing sense of comfort, safety, and control. When this cue to stop is utilized, the clinician assists the client in accessing whatever is needed to stabilize the client and to proceed with the processing. Discontinue all BLS until the client is willing and able to continue reprocessing. The clinician may explore what is needed in order that reprocessing may resume. The clinician should inquire of a client, "What happened? Why do you want to stop?" "Do you need to slow down or just take a break?" Perhaps a client needs to take a break because they are confused or overwhelmed, or they simply want to share concerns about the processing itself. The clinician may ask, "Why did you stop?" (i.e., "What stopped the train?") and address any concerns the client may have. And then, "What is it you need to continue processing?" (i.e., What does the client need in order to get back on the train?). The clinician also needs to ensure the client has what they need before continuing processing. If a client still insists that they do not want to continue, a clinician should respectfully use the procedures for closing down an incomplete session and only return to processing when a client is ready (Shapiro, 2009-2017a, 2009-2017b). The clinician may ask the client at this point to access their safe (calm) place, use a container strategy, or do some other stabilization exercise to help them to ground, to bring energy back into the body, or bring them into the present moment. The clinician should explore what the client needs to be in place in order to resume processing in the session or at the next session. If a client does not want to continue, do not implement BLS as it may reactivate the processing. Table 1.17 demonstrates some appropriate ways for a client to indicate they want to discontinue or pause the process.

It is important not to accept words for stop signals (e.g., "Stop," "No more," "I can't do this," "I want to throw in the towel," or "Whoa!") as it may be confused with what is going on in the processing.

The stop signal is tantamount to having a cord on a train the passenger can pull to indicate to the engineer that they want the train to stop for some reason. For instance, the client may need an added rest before the next stop or may want to get off the train. Ultimately, it is the engineer (i.e., clinician) who stops the train when signaled by the client in some way to stop.

At the beginning of each session, the clinician may remind the client of the chosen stop signal by saying, "If at any time you feel you have to stop, raise your hand (i.e., remind client of desired stop signal)." 


\section{TABLE 1.17 Stop Signals}

"If at any time you feel you have to stop, (remind the client of chosen stop signal)." "Is this a comfortable distance and speed?"

Hand gestures

Hand Signal

Time-Out Signal
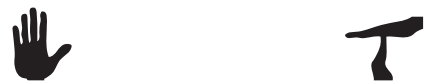

\section{Body gestures}

\section{Standing up}

Crossing arms or knees

Putting hands over mouth, ears, or eyes

Cyclist stop signal

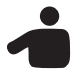

\section{EMDR THERAPY IS NOT HYPNOSIS}

A frequent question asked by clients is, "Is EMDR hypnosis?" When this question is asked, the clinician may want to spend some time with the client explaining the basic differences between the two. Clients may be concerned that EMDR reprocessing will induce a deep trance state where they may lack control. Unlike hypnosis, EMDR reprocessing causes a state of heightened emotional arousal. This is an important distinction for the clinician to make when this question arises.

The differences between hypnosis and EMDR therapy are summarized in Table 1.18.

\section{WHAT ONCE WAS ADAPTIVE BECOMES MALADAPTIVE}

Some behaviors are learned. Some serve us well and others do not. Some serve us for a period in our life and eventually become a nuisance. For example, a client who was repeatedly sexually molested by a relative as a young child may have learned to dissociate during the molestation. This was their automatic coping response to the fear and pain of the trauma at the time of the abuse. Years later, as an adult, they may still find themself dissociating during stressful situations in their work and life. As a child, dissociation was the only response available and allowed them to cope; it worked well at the time. As a maturing adult, the dissociation begins to cause problems at home, at school, and/or at work.

\section{DEVELOPING AND ENHANCING ADAPTIVE NETWORKS OF ASSOCIATION}

Before processing of the negative networks may begin, clients with more complex cases may need to access, strengthen, and reinforce positive life experiences and adaptive memories (e.g., positive resources and behaviors, learning, self-esteem). It is possible to access 
TABLE 1.18 Differences Between EMDR Therapy and Hypnosis

\section{EMDR}

Integrative psychotherapy model.

Efficacious treatment for PTSD (Bisson et al., 2007; Spates et al., 2009).

Bilateral eye movements are utilized.

EEG readings taken during EMDR reprocessing show a brain wave pattern within normal waking parameters (Nicosia, 1995).

During desensitization, the client may be in a state of heightened emotional arousal.

Clinician follows a set procedure and generally does not include cliniciangenerated suggestions (i.e., lacks suggestibility; Hekmat et al., 1994).

Each set of eye movements lasts about 30 seconds.

Memories may emerge, but memory retrieval is not the primary purpose.

Images of memories generally become more distant and less vivid, and more historical.

Clients tend to jump from one associative
memory to another.

Eyes are usually open.

\section{HYPNOSIS}

Medium within which EMDR may be practiced and enhanced (Brown, 2006).

Case reports available that suggest the efficacy of hypnosis for trauma treatment outcomes (Cardena et al., 2009).

Bilateral eye movements occur in hypnosis, but usually are ignored (Bramwell, 1906).

Theta (Sabourin et al., 1990), beta (De Pascalis \& Penna, 2009), or alpha (Meares, 1960) waves are characteristic of hypnotized subjects.

After induction, the client usually is in a deep hypnotic state.

There is no set procedure and includes clinician-generated suggestions (i.e., encourage suggestibility).

Client is in a trance lasting anywhere from 15 to 45 minutes or longer.

Is often used for memory retrieval.

Images of memories are generally enhanced and made more vivid and experienced in real time.

Clients follow a moment-by-moment ("frame by frame") sequence of events.

Eyes are closed throughout the induction and treatment phase.

Uses BLS (e.g., eye movements, taps, tones). Does not use BLS.

Clients appear more alert, remain conscious, Clients are less alert, not conscious, and and are less susceptible to inappropriate more susceptible to suggestion. suggestion.

\begin{tabular}{ll}
\hline Does not induce a trance state. & Induces a trance state. \\
\hline $\begin{array}{l}\text { Dual focus of attention is deliberately } \\
\text { maintained at all times. }\end{array}$ & $\begin{array}{c}\text { Dual focus of attention may occur } \\
\text { (Harford, 2010). }\end{array}$ \\
\hline $\begin{array}{l}\text { For long-term trauma issues, duration of } \\
\text { treatment is often brief. }\end{array}$ & $\begin{array}{c}\text { For long-term trauma issues, duration of } \\
\text { treatment is often lengthy. }\end{array}$ \\
\hline
\end{tabular}


these positive experiences by developing new and enhancing existing positive networks described as follows.

\section{Developing New Positive Networks}

(a) New positive experiences are created when the clinician teaches the client safe (calm) place and other stress management and relaxation techniques; (b) the initial introduction of BLS with stabilization exercises creates a positive experience with eye movements or other forms of BLS; and (c) the use of slower, shorter sets of BLS with the safe place, sacred space, or resource development and installation (RDI) imagery helps to create a positive experience for a client by inducing and fortifying agreeable or satisfying feelings (e.g., sense of safety, self-confidence, assurance) that currently exists within a client's positive neural networks. The goal is to create a positive experience in which a client begins to trust the BLS process, as well as strengthening and enhancing the therapeutic alliance. This may become its own positive network.

\section{Enhancing Already Existing Positive Networks}

Investigate and determine what positive life experiences and adaptive memories already exist. As the client holds these positive experiences in mind, the clinician implements BLS until the client feels the earlier positive emotions. If a client cannot come up with a positive memory, have them imagine one and facilitate the same steps mentioned previously. Identifying these positive memories is an important part of the Preparation Phase and facilitates later processing. This exercise works particularly well with a child who may need a stronger sense of safety or assurance before undergoing reprocessing.

Example: Adalia presents with a history of anxiety. The clinician asks the client to imagine a time in her past when she felt empowered and safe and instructs her to imagine how it might have felt to look, feel, and act that way again in a positive way. As she imagines this positive image, the clinician initiates short and slow sets of BLS until she can feel in the present what she believes it felt like in the imagined scene. This exercise works well with children.

Regardless of how and when they come about, it is important for these positive networks to be present and accessible for reprocessing to occur. With clients who have trouble in identifying positive networks of association or role models, plan on a longer preparation time before any processing of negative experiences.

\section{STATE VERSUS TRAIT CHANGE}

Shapiro (2008) differentiated between state and trait change. She defined a state change as momentary or transitory, whereas a trait change reflected a permanent change. A state change is a change of mind. It instills a sense of hope in the client. A state change also requires the use of coping mechanisms to continue the change, whereas a trait change no longer requires the same. With a trait change, the client changes how they see or view the event and, as a result, can experience it differently.

When a client changes their perspective about a previous traumatic event and has the needed skills, they can function more appropriately. An example of a state change is the client saying, "I am able to soothe myself by breathing and using my safe (calm) place when my boss asks me to come to his office. I feel much calmer." A trait change may be, "I am no longer triggered when my boss asks me to come to his office." To simplify, "states are weather" whereas 


\section{TABLE 1.19 State Versus Trait}

\section{STATE}

Momentary or transitory change.

Change of mind.

Coping skills may be required.

"I am able to soothe myself by breathing and using my calm/safe place when my boss asks me to come to this office. I feel much calmer."

Resourcing tends to cause state changes.

States are weather.

\section{TRAIT}

Permanent change.

Changes the way a client sees or views an event and thus can experience it differently. Coping skills are not required.

"I am no longer triggered when my boss asks me to come to his office."

Processing tends to lead to trait changes.

Traits are climate.

All traits are states, but not all states are traits.

"traits are climate." "All traits are states" but "not all states are traits" (Shapiro, 2006). See Table 1.19 for a better understanding of the differences between the two.

\section{DUAL AWARENESS-INTERNAL/EXTERNAL BALANCE}

Dual awareness or mindfulness, or what Dr. Shapiro calls "dual focus of attention" (2018), allows the client to maintain a sense of present awareness and for the client's internal processes to function without interference during reprocessing. It allows the client to be a nonevaluative observer with respect to whatever emerges during a reprocessing session. It helps the client keep "one foot in the past and one foot in the present." The client is on the "train" watching the scenery go by.

One of the primary reasons to teach a client grounding and breathing skills and anchoring them in the present is to help them learn to keep one foot in the present while reprocessing something traumatic from their past. This provides them with a dual focus of attention and reduces the possibility or risk of a client dissociating, blanking out, becoming overwhelmed, and/or resisting. Teaching them these skills prior to the reprocessing will help facilitate a smoother therapeutic experience. It also allows the client to maintain a sense of safety in the present while accessing and stimulating negative information from the past. The clinician can solidify the client's connection to the present by utilizing verbal reassurances, such as "Good," "You're doing fine," "It's over," or "You're safe now." The clinician may also stop and change the direction or speed of the BLS. When a client is in an abreactive state, these types of clinical strategies are particularly important to help the client maintain an external focus (Shapiro, 2018).

\section{ECOLOGICAL VALIDITY (I.E., SOUNDNESS)}

In attempting to discern whether a client's target has been resolved, look at what resolution of this traumatic event would look like in the real world given the individual, the timing, and the situation. To what degree does the current situation "fit" the circumstances? Ask yourself, "If a client was processing a rape that occurred months before and the rapist was still on the loose, would it be appropriate for them to continue to feel fear and demonstrate 
vigilance around this event?" The answer depends on how their information processing system works. Is there a reason they may be or may think they are still in danger? Is their sense of vigilance and fear around the rapist emotionally appropriate under the circumstances?

How does one recognize ecological validity? And how do you work with it within the EMDR therapy framework? First, use Wolpe's SUD scale (Wolpe, 1990; Exhibit 1.8).

\section{EXHIBIT 1.8 Subjective Units of Disturbance Scale}

Developed by Joseph Wolpe in 1969, the modified SUD scale is an 11-point Likert scale utilized to measure the subjective units of disturbance being experienced and reported by a client at a given time.

Neutral/No Disturbance

\section{Highest Disturbance}

\begin{tabular}{|c|c|c|c|c|c|c|c|c|c|c|}
\hline 0 & 1 & 2 & 3 & 4 & 5 & 6 & 7 & 8 & 9 & 10 \\
\hline
\end{tabular}

Abbreviation: SUD, Subjective Units of Disturbance.

The SUD scale is an 11-point Likert scale utilized to rate the anxiety level of a memory being accessed by a client in the present. When you ask the client during the Desensitization Phase to focus on the original event (incident, experience) and again ask, on a scale from 0 to 10 , "How disturbing does it feel now?" and the client says a 1, the clinician needs to check out what is blocking (i.e., blocking belief) desensitization of the original target by: (a) having the client focus on where they feel it in their body; and by asking (b) "What keeps it a 1 (or .5 or 2)?"; or (c) "What keeps it from being a 0 ?" In the case of a rapist, the client might respond, "He's still out there." Ask the client to "go with that" and continue to process to a more complete resolution. Do not assume that the client has reached the end of the channel just yet. Continue to process and check the SUD again before proceeding to the Installation Phase.

If the client still clings to the 1 (or .5 or 2) and "he's still out there," you can consider this to be ecologically valid. Ecological validity is one of the reasons the clinician should go directly to the Installation Phase without the client's SUD level getting down to a 0. The SUD scale will be discussed in more depth in Chapters 2 and 3.

A blocking belief may also arise in the Installation Phase when evaluating the VoC, a 7 -point semantic differential scale that measures the validity (i.e., felt sense of the trueness or falseness) of the client's stated PC. If the client reports a VoC of 6 or 6.5 , use the same questioning mentioned earlier when the SUD does not equal 0 (i.e., focus on where the client feels it in their body, "What keeps it a 6.5?" "What prevents it from being a 7 ?") to discern if there is: (a) a blocking belief, (b) feeder memory, or (c) ecological validity.

Dr. Shapiro has been known to say, "Forgiveness is like rain-it may or may not happen." If a client forgives someone who has hurt them, it does not mean they use poor judgment with respect to that individual (e.g., they will never leave their children with a past abuser). However, clients often arrive at forgiveness or compassion more quickly and more completely than they might with other forms of therapy. A clinician must be alert to not use 
their own experiences or experiences of their other clients to determine "ecological validity" for any specific client. If processing stops at a certain place, first attempt to remove the block by changing the direction or modality of the BLS. Always get the client's permission before doing so. Then do a couple more sets of BLS before determining if it is ecologically valid for the person to move further toward forgiveness or compassion.

\section{SIDE BENEFITS OF EMDR THERAPY}

The primary goal of EMDR therapy is to reprocess any irrational, negative cognitions; emotions; sensory stimuli (e.g., images, sounds, smells, tastes); and physical sensations associated with a trauma. It does not, however, remove or eliminate any irrational, negative sensations and cognitions related to a traumatic event. In the rape example mentioned earlier, it may be ecologically appropriate that the client maintain a healthy sense of fear and an appropriate level of vigilance until the rapist has been caught and their physical safety is ensured. It does not change the client's negative thoughts and feelings about the rapist and what they did to them.

Reprocessing will not eliminate any negative thoughts, emotions, or physical sensations that are appropriate to the situation. For example, a client may experience hate toward their abusive, neglectful, and distant mother. In response to ongoing experiences, the client may have developed low levels of self-esteem and confidence. Reprocessing may be successful in improving the client's self-esteem and other issues, but the client may or may not still feel hatred toward their mother.

EMDR therapy does not have the potential for making one fall back in love with their significant other if one does not love them, secure a raise at their job if one does not deserve one, believe that their abusiveness to another was acceptable, or make one the next race car champion of the Indy 500 if one does not have the ability to drive a race car. It cannot make the true untrue or the untrue true. It only can decompress the negative thoughts, feelings, and physical sensations from the client's internal system so that natural healing can take place. In the process of EMDR therapy, new insights may occur; behavior, perceptions, and attitudes can shift; and physical and emotional responses can change.

\section{HOLISTIC NATURE OF THE APPROACH}

Even though it has been around for more than 30 years, EMDR is very much a "cutting edge" therapy. One of the reasons that it continues to be cutting edge is that it appears to be a permanent means of flushing traumatic memories with the accompanying negative cognitions, emotions, and physical sensations from the client's system in a way that seems unique. It is a whole-system approach. It can reach down into the depths of a client's despair, attach itself to every negative element connected to a traumatic event, and then flush it out.

\section{COOKIE-CUTTER APPROACH}

EMDR therapy is not a one-size-fits-all approach. It is neither mechanistic nor cookie-cutter. Clients are as different as night and day and sessions can vary from simple to complex depending on a client's trauma history. Each client is approached individually in terms of history-taking, treatment planning, preparation, and target focus. Some clients will benefit from eye movements while others may do better with other forms of BLS. The speed and 
the length of the set will vary from client to client. Some will need more and different forms of preparation. Not only do clinicians approach each client differently, each client responds differently to EMDR treatment as well. EMDR therapy is not simply a matter of a clinician waving their fingers in front of a client's eyes as they focus on a trauma. From beginning to end, each client is treated as unique and special as the clinician helps them to navigate his way through his trauma to adaptive resolution.

\section{USEFUL METAPHORS}

\section{TRAIN METAPHOR}

While no longer a major form of transportation in America and the experience of riding a train is unfamiliar to many, the use of the train metaphor continues to be Shapiro's primary means of explaining the intricate principles, protocols, and procedures inherent in EMDR therapy.

Dr. Shapiro prescribes the use of a train metaphor to help clients move along their processing "tracks." Reference to and use of this metaphor will be utilized frequently throughout this Primer. During the Desensitization Phase, this metaphor can be applied as a means of noticing yet distancing the client from fear of the trauma. Dr. Shapiro favors this metaphor because it conveys a sense of movement and safety (Shapiro, 2006). The train metaphor may be used throughout the reprocessing as needed. It goes like this: "In order to help you just notice the experience, imagine riding on a train and the feelings, thoughts, etc., are just scenery going by" (Shapiro, 2018).

During the reprocessing, the image of the train going down the track is also used to encourage the client to continue. The passenger is the client, and the scenery represents the dysfunctional information that they are reprocessing. The clinician might say, "It's just old scenery. Just watch it go by." This metaphor is a reminder to the client that the train passes the scenery as quickly as it appears.

Dr. Shapiro describes the processing as "metaphorically like moving down a train track" (Shapiro, 2006). From the point of origination to the destination, there are freight depot stops where useless cargo (i.e., dysfunctional information) is unloaded and new, useful cargo (i.e., adaptive information) is loaded (i.e., adaptive resolution). In between stops, resources may need to be loaded for the client to continue the trip down the track, debris may need to be cleared from the track, and/or linkages to adaptive networks can occur. See Exhibit 1.9 for a pictorial rendition of this metaphor. Again, the damaged material is unloaded and discarded at the freight depots found along the track during the stopping and starting of the BLS. It is also at this freight depot where adaptive information is loaded. When the train reaches its final destination, the client has reached adaptive resolution. Metaphorically, the processing stimulated by the assessment questions and BLS move the train down the track toward adaptive resolution.

Whether suggesting the train or another example, the metaphor is an option being offered to a client if the trauma becomes too much to bear and distancing from it will allow reprocessing to continue. Installation of the metaphor is unnecessary.

During an abreaction, the train metaphor is a useful strategy for supportively assisting a client to allow movement down the tracks. The clinician can assist by saying, "It's just old information. Watch it like scenery going by."

Dr. Shapiro also uses the train metaphor to describe information that moves adaptively from dysfunctional to functional. It is a common experience for a client's once-vivid negative images, affect, and cognitions to become less vivid and less valid while the opposite happens 


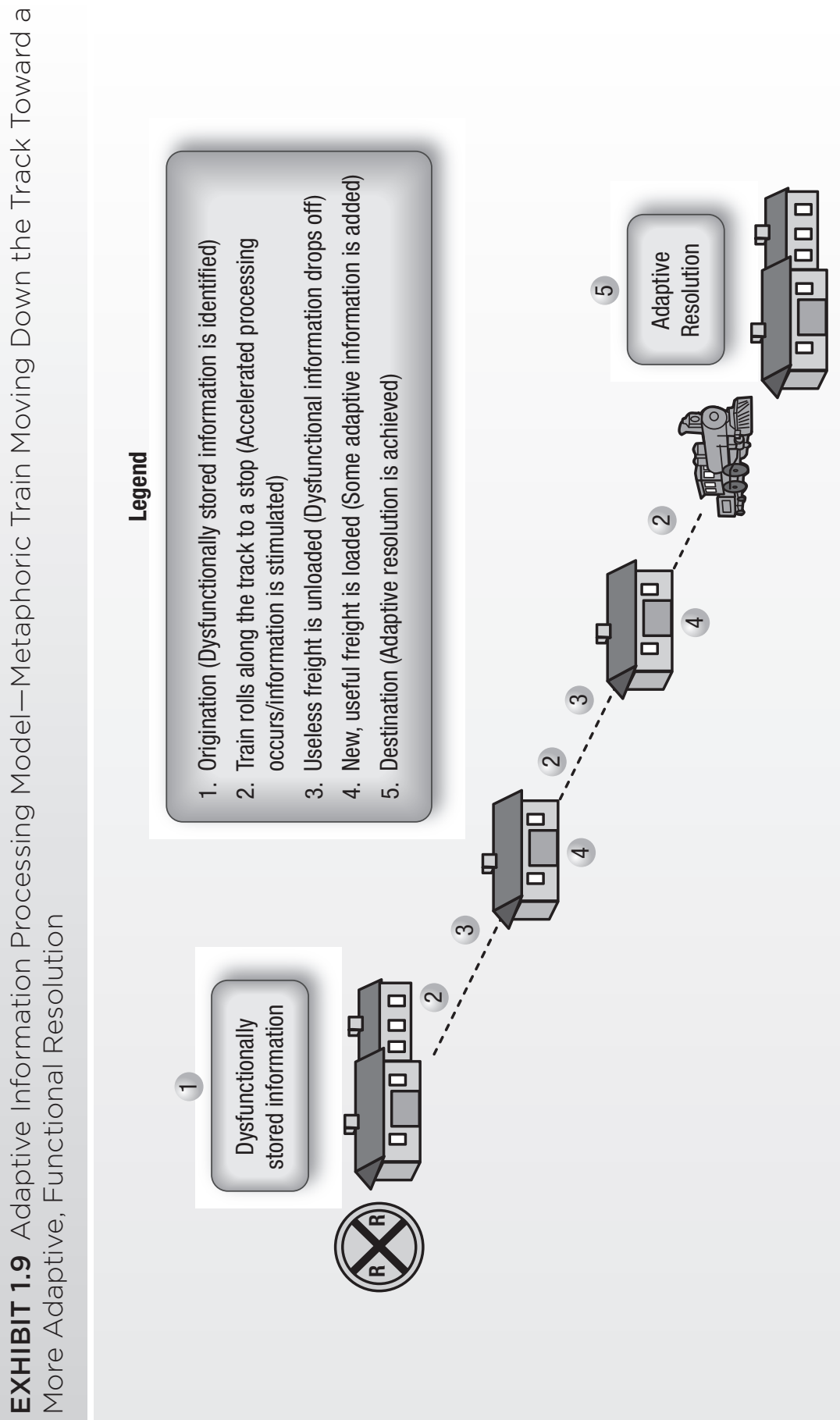


TABLE 1.20 Distancing Metaphors

Speeding train
Train
Scenery
the trauma passes by

to the positive images, affect, and cognitions. Can you visualize a train traveling down its track? Each time the dysfunctional information is stimulated or when accelerated processing takes place, the train moves down the track and stops. At each stop, the client unloads dysfunctional information and loads more functional or adaptive information. The train continues on this route until it reaches its final destination (i.e., adaptive resolution). One of the important elements of this journey that should not escape the client is that the choice to make a return trip down the same track and revisit the scenery that emerged is optional.

Although seemingly archaic, the use of the train metaphor versus others (i.e., movie theater, river) makes more sense for several reasons. It conveys the sense of movement and safety that Shapiro emphasizes (2018). It is a distancing metaphor. First, the train moves forward at a rapid rate of speed as it distances the client from the trauma while the scenery recedes in the distance at the same rate of speed. In addition, there is a visual barrier between the client who sits on the train and the scenery passing by outside the train. The protective barrier between the client and trauma helps to create a greater sense of safety. See a representation of this idea by the comparison with the other distancing metaphors in Table 1.20.

\section{TUNNEL METAPHOR}

Another metaphor used by Shapiro (2018) is driving a car through a tunnel. To get through the tunnel as quickly as possible, the driver will need to increase their pressure on the accelerator (i.e., "You are in a tunnel. Just keep your foot on the pedal and keep moving."). In EMDR reprocessing, the eye movements or other BLS act as the accelerator (i.e., the processing of the dysfunctional information is accelerated by the speed and length of the BLS). This metaphor is utilized to encourage the client to pass through the tunnel as fast as possible (i.e., keep moving his eyes quickly). If they ease up on the accelerator or choose to stop during transit, the car moves slower; and it takes much longer to get through the tunnel. Or they are left in the midst of unprocessed material. 


\section{HAND METAPHORS}

Using the hand as her prop, Shapiro explained two different aspects of the treatment process by using a metaphor. When she was training clinicians in EMDR therapy, Dr. Shapiro often used a hand metaphor to emphasize and explain the progression of the treatment effects through the memory network. With the back of her hand facing outward and her fingers pointing downward, she would explain the processing of an identified traumatic memory. The top part of the hand represented the target or node (i.e., the traumatic event or some aspect of it, such as thought, belief, body sensation) and her fingers the different channels of association that the client had to progress through to achieve adaptive resolution.

With the palm facing outward and fingers pointing downward, Dr. Shapiro would use another hand metaphor to demonstrate the different components of a memory. The top part of the hand represented the memory and the fingers the different aspects of the memory network-sensory (e.g., visual, auditory, olfactory, tactile, gustatory), thoughts, emotions, body sensations, and thoughts and beliefs (see Figure 1.1).

\section{ANCILLARY TARGETS}

A yellow or red board in train lingo is a fixed signal to slow and eventually stop a train. When an engineer encounters one of these, they know they need to stop and then, if possible, proceed with caution, depending on what is farther down the line. In some cases, there may be workers and equipment ahead indicating that the track is being repaired, or there may be an obstacle or debris blocking the tracks that needs to be cleared. Blocking beliefs (e.g., "I do not deserve good things" or "If I get over this, I will betray (be disloyal) to my buddies") and feeder memories (e.g., an unconscious memory that is emotionally feeding the targeted memory network) can activate yellow or red boards. When one of these emerges, it could be an indicator to a clinician that they may need to slow the "train" with successive sets of BLS or switch tracks to clear the track of this additional debris. Once this track has been cleared, the track is switched once again to allow the original "train" to continue down the line.

\section{SECONDARY GAINS}

Secondary-gain issues tend to obstruct or stall processing. Therefore, it is advisable for a clinician to attempt to ferret these out prior to any actual processing taking place. In the History-Taking Phase and Treatment Planning Phase, the clinician may investigate the presence of these gains by asking the client if they are aware of any reasons why processing might be unsuccessful. Is there something a client may be uncomfortable giving up to help resolve the issues they bring to therapy? What other issues are served by the presenting complaint, such as positive consequences (e.g., pension checks); needs (e.g., fear of dishonoring the dead by getting better and moving on; fear of bears may keep a wife from going on camping trips with her husband); or identity issues (e.g., fear of loss of professional or social identity)? Identify what feeds these secondary gains (e.g., low self-esteem, irrational fears, boundary issues, lack of assertiveness). Whatever the issues are, these fears need to be resolved before a successful therapeutic outcome can be expected or maintained. A clinician also needs to ensure that a client has the stability and resources available, which assists them in giving up the gains. Noncompliance may also be related to other fears (e.g., fear of success, fear of terminating therapy, fear of failure). When this occurs, the clinician may 
ask the client, "What is the worst that could happen?" or "What would change if you were successful?" The secondary-gain issues and fears may need to be addressed before any successful processing can occur.

\section{BLOCKING BELIEFS}

Blocking beliefs generally arise during EMDR processing toward the end of the Desensitization, Installation, and Body Scan phases. They become evident when a client's SUD level does not lower to a $0, \mathrm{VoC}$ does not rise to a 7 , or a clear body scan is not achieved.

\section{SUBJECTIVE UNITS OF DISTURBANCE AND THE EMERGENCE OF BLOCKING BELIEFS}

If, after repeated applications of different directions and types of BLS, the client cannot attain a 0 for a SUD level, the clinician may ask the client to: (a) focus on where they feel it in their body. If it still does not lower, the clinician is directed to look for the presence of a dysfunctional blocking belief by asking (b) "What keeps it a 1 (or .5 or 2)?" or (c) "What prevents it from being a 0 ?" If a benign or nonproblematic blocking belief (e.g., "I don't believe in absolutes") arises, the clinician should ask the client to "Just notice that," and add another set of BLS. Sometimes this allows the SUD to drop to 0. If not, then proceed to the Installation Phase.

To probe further for a blocking belief, the clinician may ask the client, "Is there a part of you that might have concerns about getting over this problem?" or "What would happen if you were to completely get over this problem?" and initiate one of more sets of BLS in order to bring forth the concern or an association to the client's consciousness. Examples of responses that may emerge are, "It's not safe to get over this problem," I don't deserve to get over this problem," or "If I feel better, I will forget about what happened."

If a dysfunctional blocking belief surfaces and, after successive sets of BLS, it does not remit, the clinician should target it with full reprocessing (i.e., Phases 3-6). This means that processing of the original target should be stopped until the blocking belief has been identified, targeted, and reprocessed. After a blocking belief has been successfully processed, the clinician should reevaluate the original targeted event and then complete the Desensitization Phase and proceed to the Installation Phase.

\section{VALIDITY OF COGNITION AND THE EMERGENCE OF BLOCKING BELIEFS}

Except for the wording, the same procedures cited earlier for blocking beliefs and the SUD level apply to blocking beliefs and the VoC. For assessing a blocking belief at the Installation Phase, the clinician asks the client: (a) to focus on where they feel it in their body; (b) "What keeps it a 6.5?"; or (c) "What prevents it from being a 7?" When assessing a client's level of validity, ecological validity should be taken into consideration by the clinician. What current life circumstances may keep a client's $\mathrm{VoC}$ from rising to a 7 ? If a client is processing a rape and their PC is, "I am safe," her 7 level may not be realistic or possible for them if their rapist is still at large. If blocking beliefs are revealed during the Installation Phase, they need to be fully processed using phases 3 through 6 . Once it has been reprocessed, the clinician should reevaluate the original targeted event and then complete the Desensitization Phase and proceed to the Body Scan Phase. 


\section{BODY SCAN AND THE EMERGENCE OF BLOCKING BELIEFS}

Blocking beliefs can emerge anywhere during EMDR treatment, including the body scan. If a blocking belief emerges during this phase and it appears more dysfunctional than innocuous, it should be targeted and processed with full reprocessing (i.e., Phases 3-6). Once resolved, the original target should be accessed, processed, and completed at the Body Scan Phase.

\section{FEEDER MEMORIES}

When Shapiro (2018) first began developing and implementing eye movement desensitization (EMD) in 1989, she started by targeting a client's current dysfunction. The result was that, when a client focused on their negative reaction to present stimuli, they would become more anxious and processing remained blocked. What she readily discovered was after several sets of BLS and often earlier, related memories would spontaneously emerge; and the client would get better once the processing of them was complete. It was then that Dr. Shapiro developed and introduced the three-pronged protocol-past, present, and futurewhereby her first area of focus changed from a client's presenting issue to the precipitating event that fueled their current dysfunction.

At this stage of development, Dr. Shapiro also began asking a client to focus on a presently held negative belief and scan back in the past for an earlier time they may have had this negative belief about themself. Because of these changes, Shapiro (2018) wrote, "The theoretical assumption of EMDR treatment is that any current dysfunctional reaction (with the exception of organically or chemically based pathologies) is always the result of a previous experience, although, of course, not necessarily one from childhood" (p. 181).

The earlier untapped memories that may emerge and appear to continue to feed a client's current dysfunction and block successful processing are called feeder memories and may arise in any of the three stages of the EMDR approach. Clinicians are now encouraged to initially treat the earlier memories with the standard protocol, followed by the present triggers and desired future outcome.

There are several ways to elicit the existence of feeder memories from a client: (a) direct questioning, (b) floatback, and (c) affect scan. If a feeder memory surfaces during the processing of another related event, these strategies may be utilized to ascertain the existence of a feeder memory within the reprocessing session or may be targeted later.

In addition to ancillary targets, there are other client obstacles that may obstruct reprocessing. In these cases, the clinician will need to determine the cause and rectify to initiate or continue processing. The obstacles highlighted in Table 1.21 were adapted from Dr. Shapiro's work (2009-2017a, 2009-2017b).

\section{TO INTERVENE OR NOT TO INTERVENE}

As a rule of thumb or when in doubt, do not intervene during reprocessing. Simply say, "Go with that." There are many indicators of successful processing, such as shifts: (a) in the memory itself; (b) from one memory to another; (c) in the reported changes or the emergence of new images, sounds, cognitive content, levels of affect, or physical sensations; (d) in a client's self-worth, self-efficacy, affect, and self-assessment; and (e) from dysfunctional to adaptive. It is important for a clinician to remember anything that emerges between sets is related to a client's experience, and only memories associated in some way with this 


\section{TABLE 1.21 Obstacles to Processing}

\begin{tabular}{ll} 
OBSTACLES & REASONS \\
$\begin{array}{c}\text { Headaches, nausea, } \\
\text { and dizziness }\end{array}$ & $\begin{array}{l}\text { Secondary-gain issues } \\
\text { May be artifact of eye movements } \\
\text { Client resistance } \\
\text { State of hypervigilance } \\
\text { May be part of the memory itself } \\
\text { May be the result of dissociation or dissociative disorder }\end{array}$ \\
\hline Client cannot feel & $\begin{array}{l}\text { Memory may be processed fully } \\
\text { Client cannot tolerate affect } \\
\text { Presently held beliefs exist that inhibit feelings (e.g., Big boys don't } \\
\text { cry/If I start to cry, I will never stop. It is dangerous, unsafe, or } \\
\text { shameful to express feelings) } \\
\text { Cultural or gender restraints or constraints with parents }\end{array}$ \\
\hline May serve as a buffer \\
Client believes he is supposed to talk
\end{tabular}

experience will emerge. To ensure the success of the reprocessing, it is imperative that a client be allowed on his own to discern the importance of the connections of all associations that may arise. As these associations arise and connections are made, the sets are continued, and the need to engage in any complex EMDR interventions is curtailed.

Table 1.22 provides some examples of changes that may occur in multimemory and single-event channels of association. When any of these shifts occur, stay out of the way and say, "Go with that."

\section{EYE MOVEMENT DESENSITIZATION VERSUS EMDR THERAPY}

In 1987, Dr. Shapiro inadvertently discovered the effects of spontaneous eye movements while focusing on disturbing thoughts of her own. She developed standardized procedures and a protocol that she called $E M D$. Two years after Dr. Shapiro's famous walk in the park, her seminal article, "Efficacy of the Eye Movement Desensitization Procedure in the Treatment of Traumatic Memories" (1989a), was published in the Journal of Traumatic Stress. In 1991, Dr. Shapiro renamed her psychotherapeutic method EMDR to recognize its shift from a desensitization paradigm to one of information processing.

While EMDR protocols are used within EMDR therapy to accomplish reprocessing of traumatic memories and comprehensive treatment, EMD is a brief strategy that can be used to reduce symptomatic reactions to a specific target or cluster of targets. EMD intentionally limits the linkages to associated memories. Associations that are reported outside of the target memory require that the client be returned to the target, the SUD reassessed, and BLS be initiated. 
TABLE 1.22 Patterns of Response

\section{CHANGES IN MULTIMEMORY EXAMPLES ASSOCIATIVE CHANNELS}

\begin{tabular}{|c|c|}
\hline $\begin{array}{l}\text { Dominant belief inherent in } \\
\text { trauma }\end{array}$ & $\begin{array}{l}\text { The memory of a boat accident brings up an } \\
\text { associated memory of being bullied and physically } \\
\text { assaulted by classmates in the second grade (both } \\
\text { shared the same negative belief, "I am powerless"). }\end{array}$ \\
\hline Major participant or perpetrator & $\begin{array}{l}\text { A client associates being beaten by his mother with } \\
\text { his mother driving drunk while he was in the car. }\end{array}$ \\
\hline Pronounced stimuli & $\begin{array}{l}\text { While processing waking up to a bedroom fire set by } \\
\text { a lit cigarette, a firefighter remembers an associative } \\
\text { memory of being overcome by smoke in a raging } \\
\text { prairie fire. }\end{array}$ \\
\hline Specific type of event & $\begin{array}{l}\text { During the processing of a memory when a teller was } \\
\text { robbed at gunpoint, an associated memory of being pistol- } \\
\text { whipped in a previous robbery years earlier emerges. }\end{array}$ \\
\hline Dominant physical sensations & $\begin{array}{l}\text { As a client processes their childhood memories of } \\
\text { being tied to a bedpost by a babysitter while their } \\
\text { parents were away, an associated memory of being } \\
\text { assaulted and tied up so the perpetrator can flee } \\
\text { spontaneously surfaces. }\end{array}$ \\
\hline Dominant emotions & $\begin{array}{l}\text { Disappointment over being passed over for a much- } \\
\text { deserved promotion is shared with an associated } \\
\text { memory of failing a CPA exam for the third time. }\end{array}$ \\
\hline $\begin{array}{l}\text { CHANGES IN SINGLE-EVENT } \\
\text { ASPECTS OF MEMORY }\end{array}$ & EXAMPLES \\
\hline Images & $\begin{array}{l}\text { Change in content or appearance: A father's outraged } \\
\text { face becomes a smiling one. } \\
\text { Change to an image of a different but associated event: } \\
\text { The image of a father's outraged face changes to } \\
\text { remembering his anger when he failed a class. } \\
\text { Change to a different aspect of the same event: The } \\
\text { image of a father's outraged face changes to one of a } \\
\text { grief-stricken man. } \\
\text { Shift in perspective: A son begins to see the image of his } \\
\text { father's outraged face as more pathetic or laughable. } \\
\text { Expansion of a scene to include more details: Original } \\
\text { image picture is one where a son sees the outrage } \\
\text { on his father's face upon hearing that his son was } \\
\text { expelled from school. As processing continued, the } \\
\text { scene opened up; and he suddenly remembered he } \\
\text { was expelled because of a physical fight that ensued } \\
\text { on school property between his father and himself. } \\
\text { The father started the fight. }\end{array}$ \\
\hline
\end{tabular}




\section{TABLE 1.22 Patterns of Response (continued)}

\section{CHANGES IN MULTIMEMORY EXAMPLES ASSOCIATIVE CHANNELS}

Shifts in the appearance of an image: The image of an outraged father may become larger (or smaller), blurs or fades, becomes closer (or more distant), turns gray or black or white, transforms into a still image, or disappears altogether.

Sounds
Cognitions

Shifts in the sound of a voice: The voice of an outraged father's voice may become softer, louder, quieter, distorted, or simply becomes mute.

Shifts in dialogue: The son suddenly commences to voice words of assertiveness toward his outraged father.

Shifts in language: The son is from Germany and reverted to his first language to express his assertiveness toward his outraged father.

Emergence of insight: As processing continues, the son suddenly realizes that the outrage his father demonstrates toward him is really about his father's sense of failure.

A polar shift occurs: In the earlier part of processing, the son's negative cognition (e.g., "I am not good enough") is replaced by a positive one (e.g., "I am okay as I am").

Emotions

Increases/decreases in intensity: After the first set of BLS, the son's fear of his outraged father becomes overwhelming (or becomes less fearful).

Shifts from one emotion to another: The son's fear of his outraged father changes to disgust, or crying changes to laughter.

Shifts toward more appropriate or ecologically valid emotions: The son's emotion shifts from fear to sadness to disgust during the course of processing.

Physical sensations

Reexperiences physical sensations tied to the emotions: The son experiences chest pains and shortness of breath as he processes fear of his father.

Experiences physical sensations felt at the time of the original event: The son feels a punch in his stomach as he processed a memory where his outraged father hit him in the gut.

Increases/decreases in intensity: The son's physical sensations of being hit in the stomach become less/ more intense during subsequent sets of BLS.

Shifts in the location: The physical sensation of being hit in the gut moves from the heart to the throat. 
TABLE 1.22 Patterns of Response (continued)

\section{CHANGES IN MULTIMEMORY EXAMPLES ASSOCIATIVE CHANNELS}

Shifts in the type of sensations felt in a certain location: The son's stomach awareness changes from nauseous to tight to feeling empty.

Abbreviations: BLS, bilateral stimulation; CPA, Certified Public Accountant.

\section{If during BLS, the client reports a free association that appears unrelated to the} precipitating event, gently say "Ok, now I would like you to go back to the bombing incident (name the event), what do you notice now?" Obtain a SUDS rating each time the client returns to the target memory. After obtaining the SUDS instruct, "Just think of that ..." (and then initiate the next set of BLS).

(RUSSELL \& FIGLEY, 2013, P. 99)

The clinician may find the use of EMD helpful when the goal is to desensitize a current or recent disturbing incident without accessing an associative memory network of experiences. This helps the client to decrease reactivity, obtain emotional regulation, and maintain dual awareness. This intervention can be used on its own or as a bridge to reprocessing. In these instances, EMD works much like other stabilization techniques, in that it helps a client establish or regain a sense of resiliency and mastery.

EMD is being used in the treatment of recent traumatic events or episodes and in several specific situations. One is when the target is an intrusive element, such as a disturbing image, sensation, thought, or feeling. Another is when processing overwhelms the AIP system. Switching briefly to EMD often helps to reduce the distress enough to start the processing again (Shapiro \& Laub, 2014).

The EMD procedure possesses the capacity to: (a) desensitize a highly disturbing or traumatic event, often within one session without an intense emotional reaction; (b) result in cognitive restructuring of the negative self-assessment along with a diminished visual representation of the original image; and (c) shift thoughts, feelings, and behaviors (Shapiro, 1989a).

Table 1.23 outlines the differences between EMD and EMDR.

The procedure initially developed by Shapiro (1989a) has evolved to more closely resemble the standard protocol, with deviations as follows (follow the standard Assessment Phase as completely as possible):

\section{ASSESSMENT PHASE}

1. Select a single memory and an image (or picture) that represents the worst part of the memory/incident.

What image (or picture) represents the worst part of the experience?

If no image/picture, When you think of the experience, what do you get?

2. Identify a negative belief (e.g., "I'm not safe," "I should have done something," "I have no control," or "I am helpless") which goes best with the target memory or image.

What words go best with the image (or picture) that express your negative belief about yourself now?

3. Identify a desired PC, such as "I'm safe now," "I did the best I could," or "It's over." 


\section{TABLE 1.23 Eye Movement Desensitization Versus EMDR}

EMD

Informed by desensitization model.

Procedure for desensitization.

Used for decreasing reactions, reducing arousal, and increasing stability to individual memories; symptom reduction while minimizing spontaneous associations to other experiences; and with clients who appear prone to emotional overwhelm, dysregulation, or outside of window of tolerance.

Brief sets of alternating eye movements or tapping facilitate effective desensitization.

\section{EMDR}

Informed by the AIP model.

Psychotherapeutic orientation.

Used for comprehensive cognitive and emotional restructuring.
Longer sets of eye movements, alternating taps and tones, facilitate information reprocessing.

Average number of passes is $12-24$.

Average recommended number of passes of BLS is $20-24$ to start, lengthen to $36-40$ as needed (the number of passes is generally customized to the client's response).

Sets are continued only as long as necessary There is no limit to the number of sets. to desensitize reactions to the target.

\section{Treatment effect}

Reduction of the fear and anxiety related to the disturbing memory.

\section{Comprehensive treatment effect}

Reduction in fear and anxiety.

Replacement of negative emotions with positive ones.

Emergence of insight.

Change in body sensations.

Surfacing of new behaviors.

Negative events transformed to adaptive learning experiences.

Abbreviations: AIP, Adaptive Information Processing; BLS, bilateral stimulation; EMD, eye movement desensitization.

When you bring up that image (or picture), what would you like (or prefer) to believe about yourself now?

4. Determine the validity of the client's PC (i.e., rate the VoC on a scale of 1-7).

When you focus on that image (or picture), how true do those words, (repeat positive cognition), feel to you now on a scale from 1 to 7 , where 1 feels completely false and 7 feels completely true?

5. Identify the associated emotions.

When you bring up that image (or picture) and those words, (repeat negative cognition), what emotion(s) do you feel now?

6. Determine the level of disturbance (i.e., rate the SUD on a scale of $0-10$ ) based on the memory or image and the negative belief. 
From 0 , which is neutral or no disturbance, to 10, which is the worst disturbance you can imagine, how disturbing does it feel to you now?

7. Identify the physical location of the body sensations.

Where do you feel it in your body?

\section{DESENSITIZATION PHASE}

8. Instruct the client to focus on the image, the negative belief, and where they feel it in their body, and initiate a set of BLS (i.e., faster, but with only 12-15 passes; set of BLS). Shorter sets of BLS are utilized with EMD to limit access to associations.

I would like you to bring up that image (or picture), those negative words (repeat negative cognition), and notice where you feel it in your body. Just let whatever happens, happen (set of BLS).

9. After each set of BLS, instruct the client to

Take a breath. (Pause.) Let it go. Then ask, What are you noticing?

The clinician should note the response, and then gently return the client to the target, NC, and reassess the SUD. After obtaining the SUD, instruct the client to "Just think of that," and initiate the next set of BLS (i.e., 12-15).

When you bring up that image (or picture) and those negative words, using a scale from 0 , which is neutral or no disturbance, to 10, which is the worst disturbance you can imagine, how disturbing does it feel to you now?

10. Repeat this process until the client reports little or no disturbance (i.e., SUD $=0$ or ecologically sound) and no new intrusive symptoms emerge.

11. Once the SUD has stopped decreasing, the clinician moves to the Installation Phase. When you focus on that image (or picture), do the words, (repeat positive cognition), still fit, or is there another positive statement you feel would be more suitable? When you focus on that image (or picture), how true do those words, (repeat positive cognition), feel to you now on a scale from 1 to 7 , where 1 feels completely false and 7 feels completely true?

Hold the two together. The clinician initiates BLS.

On a scale from 1 to 7 , how true do those words, (repeat positive cognition), feel to you now when you focus on the original experience?

The clinician continues to install the PC as long as the reprocessing keeps becoming more and more adaptive. If the reported $\mathrm{VoC}$ does not increase to 7 , the clinician will check for ecological appropriateness. The existence of blocking beliefs should be considered.

Continue to the Closure phase. See Chapter 6 for a complete transcript using EMD with a client. Please note that a Body Scan is not initiated using EMD as the clinician does not want to run the risk of opening up associative links.

\section{PRACTICAL TIPS TO REMEMBER}

\section{PRACTICE, PRACTICE, PRACTICE}

Practice, practice, practice is this Primer's mantra. In the EMDR Weekend 1 and 2 Trainings you were introduced to EMDR therapy-but, because of time limitations, you may not have fully integrated its substance and protocol into your own therapeutic paradigm. Learning EMDR therapy comes from the actual doing of it. Even skilled clinicians who have conducted 
hundreds of sessions have the potential for learning something new every time they execute the process with a client. It is only from practicing EMDR that excellence and expertise can be derived; so, the mantra practice, practice, practice cannot be overly emphasized.

\section{FOLLOW THE SCRIPT VERBATIM}

Newly trained EMDR therapy clinicians are strongly encouraged to follow Dr. Francine Shapiro's script verbatim in the Assessment Phase. Dr. Shapiro has chosen every word for a specific reason, and these words have been tested and validated repeatedly in one context or another in session after session with clients presenting various mental health issues. It is important for the clinician to understand the intent and implications of the wording of the well-researched protocol before implementing individual styles of eliciting the same information. In addition, it is highly beneficial for clinicians to follow the scripts provided for Phases 4 through 6. Clinicians who learned the script in the early days of EMDR therapy may notice how it has been refined throughout the past 30 years.

If you have been recently trained or have decided to finally put your EMDR training to use, sit with a copy of the standard protocol in your lap as you implement the Assessment Phase with clients (see Chapter 3). Reading the script verbatim may feel unnatural at first, but you can expect to feel more at ease as you learn the procedural steps. Sitting with the pages in your lap and reading the script as it is written can also serve as good modeling for your client as they watch you work with something new on their behalf. As they become more familiar with the protocol and what words are required in each part, the clinician will most likely develop their own style for setting up the EMDR protocol. The words in the Assessment Phase necessary to optimize receiving the desired processing outcome have been underlined in this chapter and in subsequent ones for your recognition and convenience.

The EMDR Fidelity Rating Scale (EFRS) was developed by van der Kolk et al. (2007) to evaluate adherence to EMDR therapy's eight phases of treatment (i.e., Phases 1-8) and three-pronged protocol (i.e., past, present, future). The EFRS can help a clinician evaluate use of the standard EMDR therapy protocol with memories associated with adverse life events or current triggers. A modified version of this scale (Korn et al., 2018) can be found on the EMDR Research Foundation website (see https://emdrfoundation.org/researchgrants/emdr-fidelity-rating-scale/). Clinicians are strongly encouraged to become familiar with this helpful scale. The more fidelity a clinician demonstrates, the better treatment outcome for the client.

Consider logging onto the Trauma Recovery/EMDR Humanitarian Assistance Programs (TR/EMDR-HAP) website to contribute to a worthy cause. You may want to consider purchasing the laminated SUD/VoC Scale Chart or the EMDR Progress Notepad. These items may be purchased online at the TR/EMDR-HAP website (https://www.emdrhap.org/content/). The worksheets can assist you in being more consistent and successful from client to client. The laminated chart may save time and help the client to distinguish a belief from a feeling and to select a negative belief appropriate to their situation. It is not an uncommon reaction for a client to look like a deer caught in the headlights when asked, "What words go best with the picture that express your negative belief about yourself now?" Having said this, use this placard sparingly. In all cases, it is imperative that the client be given ample opportunity to provide the clinician with a negative and positive cognitions on their own. When a client seemingly does not understand what is being asked for, the clinician should be knowledgeable and skilled in ways to tease these cognitions out before handing them the placard. 


\section{KNOW YOUR CLIENT}

Before you begin using the reprocessing phases, it is important that you know your client well. Know their strengths and weaknesses. Know their abilities and their limitations. Know their ego deficits. Know their coping mechanisms and strategies. Know their support system - or lack of it. Some clients may not be appropriate or ready for EMDR trauma processing. There could be situations, however, in which you will not have the luxury of waiting weeks to know your client before beginning reprocessing. Then it becomes imperative that you gain as much information as you can about your client in a brief period, particularly when situations or circumstances indicate a necessity of serious caution.

\section{STAY OFF THE TRACKS}

The hallmark of EMDR reprocessing is facilitating a client's flow of association and allowing the client to get to the end of the "track" on their own. After completion of the Assessment Phase, the clinician is encouraged to be very limited in what they say, such as "Take a breath. (Pause.) Let it go." "What are you noticing now?" "Good." "Go with that." "Notice that." The clinician does not say much of anything else unless the client appears stuck in the process.

The most appropriate and easiest method to stay out of the way is by consistently maintaining a position of quiet neutrality. During the process, the clinician encourages the client by saying "Good" or "You're doing fine." Beyond this, the clinician must be careful not to physically or verbally express what they believe or think about a client's responses between sets of BLS. It is imperative that the clinician allow the client to own the reprocessing of their traumatic event and not be encumbered by the clinician's interventions, comments, or questions. Remember, the clinician is not the agent of change: the client is.

\section{TRACKING THE CLIENT}

It is important for the clinician to write down as much as possible of what the client says during the Assessment Phase, especially the exact wording of the client's negative and positive cognitions and key words from their descriptions of traumatic events. Why? Because it is important to use exact wording when activating what the client says. If a client provides a negative cognition, such as "It's my fault," and a clinician reframes it as "I'm responsible," the clinician may have inadvertently distorted what the client originally meant. In doing so, the clinician has also placed themself in the client's process. Because the clinician reframed it that way, the client may begin to interpret it as "I'm responsible," simply because the clinician said it. "It's my fault" and "I am responsible" may or may not mean the same to the client. To the degree that it does not, it can alter the direction of processing. During the remaining reprocessing Phases 4 through 6, clinicians often find it helpful to write down what the client says. However, if writing down what the client says during reprocessing slows, interrupts, or hinders in any way the client's flow, stop writing and opt to listen and observe more closely what the client is experiencing in the moment.

\section{KEEP IT SIMPLE}

In the early days of your EMDR experience as a clinician, try to keep it simple. Do not go straight from the training to your office and select the most challenging client to conduct your first reprocessing session. Select someone with a less complex trauma, such as a client 
who presents with a single-event trauma. Maybe someone has recently been involved in an automobile accident that relates to no other traumatic event in their life. As will be described in Chapter 4, when the three-pronged approach is discussed, multiple-event traumas are more comprehensive, will take a longer time frame to deal with, and require more skill than a client who presents with a single event. As a new EMDR clinician, you may not yet have the skill level required to deal with multiple-event traumas.

\section{POWER OF NOW}

One of the most emphasized words in the EMDR protocols is "now." Why? Because we are asking the client what they believe negatively about themself, what they want to believe positively about themself, and what are the negative emotions and physical sensations that go with the event they are focusing on "now." How are they being affected in the present by something that happened to them 2 months, 2 years, or 20 years ago? How are they being affected now?

The clinician may need to repeat the "now" over and over to a client. The client may get confused between how they felt "then" about an incident and how they feel "now" and ask questions that indicate their confusion. "Do you mean then or now?" And they could say, "Then it felt awful, but now it does not feel so bad." If this happens during the Assessment Phase, the clinician may need to reevaluate whether the client has chosen an appropriate target. Remember, the clinician is looking to relieve the client of a memory that is charged with negativity. Use Exhibit 1.10 to help remember this important point.

\section{EXHIBIT 1.10 Past, Present, Future}

\section{How we feel and react to something in the}

present

that happened in the

past

that we do not want to happen in the

future

\section{ONE MORE TIME}

A good rule to remember during EMDR reprocessing with a client is that any time something is positively reinforced with BLS, it strengthens the focus of reinforcement. So, when 
a client reports a positive direction in the reprocessing, say "Go with that" just one more time before returning to target. After the client reaches the SUD of $0, \mathrm{VoC}$ of 7 , and a clear Body Scan, say "Go with that" one or more times to reinforce the positive treatment effect and/or to allow deepening of the PC (Shapiro, 2018, 2009-2017a, 2009-2017b). If the clinician is consistent with this rule, the success of the EMDR will be enhanced. In any case, it is important to continue BLS if positive material continues to emerge or strengthen in any part of the client's reprocessing experience.

When reinforcing a positive effect during the Desensitization, Installation, and Body Scan Phases, the BLS will be faster and the length of the sets longer (i.e., 20-24 round-trip passes to start, lengthen to $30-40$ as needed) than during the Preparation Phase, when using the safe (calm) place and other resource-building exercises (i.e., slower 4-6 round-trip passes). As indicated earlier, slower and shorter sets are utilized in stabilization efforts to not activate any disturbing material prior to actual reprocessing with EMDR. The primary reason for utilizing faster, longer sets during Phases 3 through 6 is that negative associations may emerge at any phase. Faster, longer sets facilitate the resolution of the negative material, adaptive resolution, and generalization.

\section{SOLO RUN}

Client selection is always an important part of the EMDR therapy process, but more so when a clinician is choosing clients for a first solo run. Clinicians may want to select clients with whom they have a strong client-clinician relationship. Pick the less complicated cases. Think of a client's trauma as an onion. How many layers are there? How thick? How thin? So, when looking at the onion, look for the one- or thin-layered skins. And go slowly.

If you are new to EMDR therapy, select a client with more strengths than weaknesses, adequate coping mechanisms, and a supportive network of family and friends. Initially, you may also want to consider working with a client's lesser issues to build up your experience and the client's confidence in AIP during your learning process. For example, Sharon entered therapy 3 weeks prior. She had been sexually abused over a long period of time by an older brother who had an intellectual disability. Because Sharon was new to therapy and the clinician was new to EMDR, it was decided that her first reprocessing session would focus on her fear of dogs. It turned out that Sharon had been bitten by a stray dog at the age of 5 years. Because the session was so successful, they were able to continue using the process on the sexual abuse she experienced at the hands of her older brother.

Dr. Shapiro suggests first identifying the "touchstone" event when there may be one (2008). Before proceeding in this direction, however, one must carefully discern whether it is possible for the client to attain successful processing of a touchstone event. This means that the client must be able to tolerate any level of disturbance that may arise. If the touchstone event is chosen for processing and the client becomes too overwhelmed by the experience, much time could be lost by having to "undo" the client's newly created fear of the process. On the other hand, if you do not target the touchstone event and it arises as a feeder memory, it has the potential to be even more disturbing. The significance of completing a comprehensive history and obtaining informed consent becomes clearer here; that is, it is important for the client to be informed of the potential for accessing feeder memories during the reprocessing of any chosen target.

In this chapter, an attempt has been made to help refamiliarize the reader with basic concepts inherent in EMDR and/or to provide valuable updates to EMDR therapy. Throughout 
subsequent chapters, case examples will be provided along with teaching points that attempt to explain the clinician's strategies or to point out techniques prescribed by Dr. Shapiro during reprocessing.

\section{SUMMARY STATEMENTS}

1. EMDR therapy is an integrative psychotherapeutic approach and is guided by the AIP model. The AIP model "provides the theoretical framework and principles for treatment and an explanation of the basis of pathology and personality development" (Shapiro, 2018). As an integrative psychotherapeutic approach, EMDR therapy is distinct from cognitive behavioral therapy, experiential, and psychodynamic approaches, although it is not exclusive and may be informed by or used together with these approaches.

2. EMDR therapy has eight distinct phases.

3. EMDR therapy is a three-pronged approach addressing the past, the present, and the future.

4. BLS is not EMDR therapy. It is only one component.

5. During the active reprocessing phases of EMDR therapy, dual awareness should be maintained always: one foot in the present, and one foot in the past.

6. EMDR therapy is a fluid, dynamic approach that entails the clinician using all their clinical skills. It is neither mechanistic nor a cookbook approach.

7. The heart of EMDR therapy is the AIP model. As such, it is critical that the clinician have a clear understanding of it to proceed with EMDR practice.

8. Practice, practice, practice. This is how we learn the model.

9. Know your client thoroughly.

10. Stay out of the client's way. The reprocessing is about the client, not the clinician.

\section{REFERENCES}

Acierno, R., Tremont, G., Last, C., \& Montgomery, D. (1994). Tripartite assessment of the efficacy of eye-movement desensitization in a multiphobic patient. Journal of Anxiety Disorders, 8, 259-276. https://doi.org/10.1016/0887-6185(94)90007-8

Adler-Tapia, R. (2015). EMDR psychotherapy case conceptualization with a reverse protocol [Conference presentation]. Twentieth EMDR International Association Conference, Philadelphia, PA.

Afifi, T. O., Mota, N. P., Dasiewicz, P., MacMillan, H. L., \& Sareen, J. (2012). Physical punishment and mental disorders: Results from a nationally representative US sample. Pediatrics, 130(2), 184-192. https://doi.org/10.1542/peds.2011-2947

Allon, M. (2015). EMDR group therapy with women who were sexually assaulted in the Congo. Journal of EMDR Practice and Research, 9, 28-34. https://doi.org/10.1891/1933-3196.9.1.28

American Psychiatric Association. (2013). Diagnostic and statistical manual of mental disorders (5th ed.). Author.

Andrade, J., Kavanagh, D., \& Baddeley, A. (1997). Eye-movements and visual imagery: A working memory approach to the treatment of posttraumatic stress disorder. British Journal of Clinical Psychology, 36, 209-223. https://doi.org/10.1111/j.2044-8260.1997.tb01408.x

Armstrong, M. S., \& Vaughan, K. (1996). An orienting response model of eye movement desensitization. Journal of Behavior Therapy \& Experimental Psychiatry, 27, 21-32. https://doi. org/10.1016/0005-7916(95)00056-9 
Arseneault, L., Cannon, M., Fisher, H. L., Polanczyk, G., Moffitt, T. E., \& Caspi, A. (2011). Childhood trauma and children's emerging psychotic symptoms: A genetically sensitive longitudinal cohort study. American Journal of Psychiatry, 168, 65-72. https://doi.org/10.1176/appi. ajp.2010.10040567

Bae, H., Kim, D., \& Park, Y. C. (2008). Eye movement desensitization and reprocessing for adolescent depression. Psychiatry Investigation, 5(1), 60-65. https://doi.org/10.4306/pi.2008.5.1.60

Barrowcliff, A. L., Gray, N. S., Freeman, T. C. A., \& MacCulloch, M. J. (2004). Eye-movements reduce the vividness, emotional valence and electrodermal arousal associated with negative autobiographical memories. Journal of Forensic Psychiatry and Psychology, 15(2), 325-345. https://doi. org/10.1080/14789940410001673042

Barrowcliff, A. L., Gray, N. S., MacCulloch, S., Freeman, T. C., \& MacCulloch, M. J. (2003). Horizontal rhythmical eye movements consistently diminish the arousal provoked by auditory stimuli. British Journal of Clinical Psychology, 42(Pt 3), 289-302. https://doi.org/10.1348/01446650360703393

Behnam Moghadam, M., Alamdari, A. K., Behnam Moghadam, A., \& Darban, F. (2015). Effect of EMDR on depression in patients with myocardial infarction. Global Journal of Health Science, 7 , 258-262. https://doi.org/10.5539/gjhs.v7n6p258

Bergmann, U. (2000). Further thoughts on the neurobiology of EMDR: The role of the cerebellum in accelerated information processing. Traumatology, 6(3), 175-200. https://doi.org/ $10.1177 / 153476560000600303$

Bergmann, U. (2008). The neurobiology of EMDR: Exploring the thalamus and neural integration. Journal of EMDR Practice and Research, 2(4), 300-314. https://doi.org/10.1891/1933-3196.2.4.300

Bergmann, U. (2010). EMDR's neurobiological mechanisms of action: A survey of 20 years of searching. Journal of EMDR Research and Practice, 4, 22-42. https://doi.org/10.1891/1933-3196.4.1.22

Bisson, J. I., Ehlers, A., Matthews, R., Pilling, S., Richards, D., \& Turner, S. (2007). Psychological treatments for chronic posttraumatic stress disorder. Systematic review and meta-analysis. British Journal, 190, 97-104. https://doi.org/10.1192/bjp.bp.106.021402

Boudewyns, P. A., \& Hyer, L. A. (1996). Eye movement desensitization and reprocessing (EMDR) as treatment for posttraumatic stress disorder (PTSD). Clinical Psychology and Psychotherapy, 3, 185-195. https://doi.org/10.1002/(SICI)1099-0879(199609)3:3<185::AID-CPP101>3.0.CO;2-0

Bramwell, J. M. (1906). Hypnotism. Alexander Moring, Ltd.

Brown, D. (2006). Treatment of attachment pathology in patients with trauma-related diagnoses. Workshop presented at the Annual Trauma Conference, Harvard Medical School, Boston, MA.

Brown, K. W., McGoldrick, T., \& Buchanan, R. (1997). Body dysmorphic disorder: Seven cases treated with eye movement desensitization and reprocessing. Behavioural and Cognitive Psychotherapy, 25(2), 203-207. https://doi.org/10.1017/S1352465800018403

Brown, S., \& Shapiro, F. (2006). EMDR in the treatment of borderline personality disorder. Clinical Case Studies, 5(5), 403-420. https://doi.org/10.1177/1534650104271773

Calancie, O. G., Khalid-Khan, S., Booij, L., \& Munoz, D. P. (2018). Eye movement desensitization and reprocessing as a treatment for PTSD: Current neurobiological theories and a new hypothesis. Annals of the New York Academy of Science, 1426(1), 127-145. https://doi.org/10.1111/nyas.13882

Cardena, E., Maldonado, J., van der Hart, O., \& Spiegel, D. (2009). Hypnosis. In E. B. Foa, T. M. Keane, \& M. J. Friedman (Eds.), Effective treatments for PTSD: Practice guidelines from the International Society for Traumatic Stress Studies (2nd ed., pp. 427-457). Guilford Press.

Chemtob, C. M., Tolin, D. F., van der Kolk, B. A., \& Pitman, R. K. (2000). Eye movement desensitization and reprocessing. In E. B. Foa, T. M. Keane, \& M. J. Friedman (Eds.), Effective treatments for PTSD: Practice guidelines from the International Society for Traumatic Stress Studies (pp. 139-155, 333-335). Guilford Press.

Christman, S. D., Garvey, K. J., Propper, R. E., \& Phaneuf, K. A. (2003). Bilateral eye movements enhance the retrieval of episodic memories. Neuropsychology, 17, 221-229. https://doi.org/10.3758/ PBR.15.3.515

Christman, S. D., Propper, R. E., \& Brown, T. J. (2006). Increased interhemispheric interaction is associated with earlier offset of childhood amnesia. Neuropsychology, 20, 336. https://doi. org/10.1037/0894-4105.20.3.336 
Davidson, P. R., \& Parker, K. C. H. (2001). Eye movement desensitization and reprocessing (EMDR): A meta-analysis. Journal of Consulting and Clinical Psychology, 69, 305-316. https://doi. org/101037//0022-006x.69.2.305

De Jongh, A., Ernst, R., Marques, L., \& Hornsveld, H. (2013). The impact of eye movements and tones on disturbing memories involving PTSD and other mental disorders. Journal of Behavior Therapy and Experimental Psychiatry, 44(4), 477-483. https://doi.org/10.1016/j.jbtep.2013.07.002

De Pascalis, V., \& Penna, P. M. (2009). 40-Hz EEG activity during hypnotic induction and hypnotic testing. International Journal of Clinical and Experimental Hypnosis, 38(2), 125-138. https://doi. org/10.1080/00207149008414507

de Roos, C., Veenstra, A., de Jongh, A., den Hollander-Gijsman, M., van der Wee, N., Zitman, F., \& van Rood, Y. R. (2010). Treatment of chronic phantom limb pain using a trauma-focused psychological approach. Pain Research \& Management, 15(2), 65-71. https://doi.org/10.1155/ 2010/981634

Devilly, G. J., Spence, S. H., \& Rapee, R. M. (1998). Statistical and reliable change with eye movement desensitization and reprocessing: Treating trauma with a veteran population. Behavior Therapy, 29, 435-455. https://doi.org/10.1016/S0005-7894(98)80042-7

Doering, S., Ohlmeier, M.-C., de Jongh, A., Hofmann, A., \& Bisping, V. (2013). Efficacy of a traumafocused treatment approach for dental phobia: A randomized clinical trial. European Journal of Oral Sciences, 121(6), 584-593. https://doi.org/10.1111/eos.12090

El Khoury-Malhame, M., Lanteaume, L., Beetz, E. M., Roques, J., Reynaud, E., Samuelian, J. C., Blin, O, Garcia, R., \& Khalfa, S. (2011). Attentional bias in post-traumatic stress disorder diminishes after symptom amelioration. Behavior Research and Therapy, 49(11), 796-801. https://doi. org/10.1016/j.brat.2011.08.006

Elofsson, U. O., von Scheele, B., Theorell, T., \& Sondergard, H. P. (2008). Physiological correlates of eye movement desensitization and reprocessing. Journal of Anxiety Disorders, 22(4), 622-634. https://doi.org/10.1016/j.janxdis.2007.05.012

Engelhard, I. M., van den Hout, M. A., Dek, E. C. P., Giele, C. L., van der Wielen, J.-W., Reijnen, M. J., \& van Roij, B. (2011). Reducing vividness and emotional intensity of recurrent "flashforwards" by taxing working memory: An analogue study. Journal of Anxiety Disorders, 25, 599-603. https://doi.org/10.1016/j.janxdis.2011.01.009

Engelhard, I. M., van den Hout, M. A., Janssen, W. C., \& van der Beek, J. (2010a). Eye movements reduce vividness and emotionality of "flashforwards". Behaviour Research and Therapy, 48, 442-447. https://doi.org/10.1016/j.brat.2010.01.003

Engelhard, I. M., van den Hout, M. A., Janssen, W. C., \& van der Beek, J. (2010b). The impact of taxing working memory on negative and positive memories. European Journal of Psychotraumatology, 1, 5623. https://doi.org/10.3402/ejpt.vli0.5623

Faretta, E. (2013). EMDR and cognitive behavioral therapy in the treatment of panic disorder: A comparison. Journal of EMDR Practice and Research, 7, 121-133. https://doi.org/ 10.1891/1933-3196.7.3.121

Felitti, V. J., Anda, R. F., Nordenberg, D., Williamson, D. F., Spitz, A. M., Edwards, V., Koss, M. P., \& Marks, J. S. (1998). Relationship of childhood abuse and household dysfunction to many of the leading causes of death in adults: The adverse childhood experiences (ACE) study. American Journal of Preventive Medicine, 14, 245-258. https://doi.org/10.1016/S0749-3797(98)00017-8

Fernandez, I., \& Faretta, E. (2007). Eye movement desensitization and reprocessing in the treatment of panic disorder with agoraphobia. Clinical Case Studies, 6(1), 44-63. https://doi.org/ $10.1177 / 1534650105277220$

Flannery, R., Jr. (1995). Posttraumatic stress disorder: The victim's guide to healing \& recovery. Crossroad.

Friedman, D., Goldman, R., Stern, Y., \& Brown, T. (2009). The brain's orienting response: An eventrelated functional magnetic resonance imaging investigation. Human Brain Mapping, 30(4), 1144-1154. https://doi.org/10.1002/hbm.20587

Gauhar, M., \& Wajid, Y. (2016). The efficacy of EMDR in the treatment of depression. Journal of EMDR Practice and Research, 10(2), 59-69. https://doi.org/10.1891/1933-3196.10.2.59 
Gauvreau, P., \& Bouchard, S. (2008). Preliminary evidence for the efficacy of EMDR in treating generalized anxiety disorder. Journal of EMDR Practice and Research, 2(1), 26-40. https://doi. org/10.1891/1933-3196.2.1.26

Gold, S. D., Marx, B. P., Soler-Baillo, J. M., \& Sloan, D. M. (2005). Is life stress more traumatic than traumatic stress? Journal of Anxiety Disorders, 19, 687-698. https://doi.org/10.1016/j. janxdis.2004.06.002.

Grand, D., \& Goldberg, A. (2011). This is your brain on sports: Beating blocks, slumps and performance anxiety for good! Dog Ear Publishing.

Gunter, R. W., \& Bodner, G. E. (2008). How eye movements affect unpleasant memories: Support for a working-memory account. Behaviour Research and Therapy, 46(8), 913-931. https://doi. org/10.1016/j.brat.2008.04.006

Harford, P. M. (2010). The integrative use of EMDR and clinical hypnosis in the treatment of adults abused as children. Journal of EMDR Practice and Research, 4(2), 60-75. https://doi. org/10.1891/1933-3196.4.2.60

Heim, C., Plotsky, P. M., \& Nemeroff, C. B. (2004). Importance of studying the contributions of early adverse experience to neurobiological findings in depression. Neuropsychopharmacology, 29, 641-648. https://doi.org/10.1038/sj.npp.1300397

Heins, M., Simons, C., Lataste, T., Pfeifer, S., Versmissen, D., Lardinois, M., Marcelis, M., Delespaul, P., Krabbendam, L., van Os, J., Myin-Germeys, I. (2011). Childhood trauma and psychosis: A case-control and case-sibling comparison across different levels of genetic liability, psychopathology, and type of trauma. American Journal of Psychiatry, 168, 1286-1294. https://doi. org/10.1176/appi.ajp.2011.10101531.

Hekmat, H., Groth, S., \& Rogers, D. (1994). Pain ameliorating effect of eye movement desensitization. Journal of Behavior Therapy and Experimental Psychiatry, 25(2), 121-129. https://doi. org/10.1016/0005-7916(94)90004-3

Hofmann, A. (2009). The inverted EMDR standard protocol for unstable complex post-traumatic stress disorder. In M. Luber (Ed.), Eye movement desensitization (EMDR) scripted protocols: Special populations (pp. 313-328). Springer Publishing Company.

Homer, S. R., Deeprose, C., \& Andrade, J. (2016). Negative mental imagery in public speaking anxiety: Forming cognitive resistance by taxing visuospatial working memory. Journal of behavior therapy and experimental psychiatry, 50, 77-82. https://doi.org/10.1016/j.jbtep.2015. 05.004

Hornsveld, H. K., Landwehr, F., Stein, W., Stomp, M. P. H., Smeets, M. A. M., \& van den Hout, M. A. (2010). Emotionality of loss-related memories is reduced after recall plus eye movements but not after recall plus music or recall only. Journal of EMDR Practice and Research, 3(4), 106-112. https://doi.org/10.1891/1933-3196.4.3.106

Kapoula, Z., Yang, Q., Bonnet, A., Bourtoire, P., \& Sandretto, J. (2010). EMDR effects on pursuit eye movements. PLoS ONE, 5(5), e10762. https://doi.org/10.1371/journal.pone.0010762

Kavanagh, D. J., Freese, S., Andrade, J., \& May, J. (2001). Effects of visuospatial tasks on desensitization to emotive memories. British Journal of Clinical Psychology, 40, 267-280. https://doi. org $/ 10.1348 / 014466501163689$

Kearns, M., \& Engelhard I. M. (2015). Psychophysiological responsivity to script-driven imagery: An exploratory study of the effects of eye movements on public speaking flashforwards. Frontiers in Psychiatry, 6, 115. https://doi.org/10.3389/fpsyt.2015.00115

Kip, K. E., Sullivan, K. L., Lengacher, C. A., Rosenzweig, L., Hernandez, D. F., Kadel, R., Kozel, F. A., Shuman, A., Girling, S. A., Hardwick, M. J., \& Diamond, D. M. (2013). Brief treatment of co-occurring post-traumatic stress and depressive symptoms by use of accelerated resolution therapy. Frontiers in Psychiatry, 4(11). https://doi.org/10.3389/fpsyt.2013.00011

Korn, D. L., Maxfield, L., Stickgold, R., \& Smyth, N. J. (2018). EMDR Fidelity Rating Scale (EFRS), version 2. https://emdrresearchfoundation.org/research-grants/emdr-fidelity-rating-scale/

Kristjánsdóttir, K., \& Lee, C. W. (2011). A comparison of visual versus auditory concurrent tasks on reducing the distress and vividness of aversive auto-biographical memories. Journal of EMDR Practice and Research, 5(2), 34-41. https://doi.org/10.1891/1933-3196.5.2.34 
Kuiken, D., Bears, M., Miall, D., \& Smith, L. (2002). Eye movement desensitization reprocessing facilitates attentional orienting. Imagination, Cognition and Personality, 21(1), 3-20. https://doi. org/10.2190/L8JX-PGLC-B72R-KD7X

Kuiken, D., Chudleigh, M., \& Racher, D. (2010). Bilateral eye movements, attentional flexibility and metaphor comprehension: The substrate of REM dreaming? Dreaming, 20, 227-247. https://doi. org/10.1037/a0020841

Lee, C. W., \& Cuijpers, P. (2013). A meta-analysis of the contribution of eye movements in processing emotional memories. Journal of Behavior Therapy and Experimental Psychiatry, 44(2), 231-239. https://doi.org/10.1016/j.jbtep.2012.11.001

Lee, C. W., \& Drummond, P. D. (2008). Effects of eye movement versus therapist instructions on the processing of distressing memories. Journal of Anxiety Disorders, 22(5), 801-808. https://doi. org/10.1016/j.janxdis.2007.08.007

Lee, C. W., Taylor, G., \& Drummond, P. D. (2006). The active ingredient in EMDR: Is it traditional exposure or dual focus of attention? Clinical Psychology and Psychotherapy, 13(2), 97-107. https:// doi.org/10.1002/cpp.479

Leer, A., Engelhard, I. M., \& van den Hout, M. A. (2014). How eye movements in EMDR work: Changes in memory vividness and emotionality. Journal of Behavior Therapy and Experimental Psychiatry, 45(3), 396-401. https://doi.org/10.1016/j.jbtep.2014.04.004

Lilley, S. A., Andrade, J., Turpin, G., Sabin-Farrell, R., \& Holmes, E. A. (2009). Visuospatial working memory interference with recollections of trauma. British Journal of Clinical Psychology, 48, 309-321. https://doi.org/10.1348/014466508X398943

Lipke, H. (1999). Comments on "thirty years of behavior therapy" and the promise of the application of scientific principles. The Behavior Therapist, 22, 11-14.

Lohr, J. M., Tolin, D. F., \& Kleinknecht, R. A. (1995). An intensive investigation of eye movement desensitization of medical phobias. Journal of Behavior Therapy and Experimental Psychiatry, 26, 141-151. https://doi.org/10.1016/0887-6185(95)00036-4

Lohr, J. M., Tolin, D. F., \& Kleinknecht, R. A. (1996). An intensive investigation of eye movement desensitization of claustrophobia. Journal of Anxiety Disorders, 10, 73-88. https://doi. org/10.1016/0887-6185(95)00036-4

MacCulloch, M. (2006). Effects of EMDR on previously abused child molesters: Theoretical reviews and preliminary findings from Ricci, Clayton, and Shapiro. Journal of Forensic Psychiatry and Psychology, 17(4), 531-537. https://doi.org/10.1080/14789940601075760

MacCulloch, M. J., \& Feldman, P. (1996). Eye movement desensitisation treatment utilises the positive visceral element of the investigatory reflex to inhibit the memories of post-traumatic stress disorder: A theoretical analysis. British Journal of Psychiatry, 169(5), 571-579. https://doi. org/10.1192/bjp.169.5.571

Madrid, A., Skolek, S., \& Shapiro, F. (2006). Repairing failures in bonding through EMDR. Clinical Case Studies, 5(4), 271-286. https://doi.org/10.1177/1534650104267403

Maxfield, L., Melnyk, W. T., \& Hayman, C. A. G. (2008). A working memory explanation for the effects of eye movements in EMDR. Journal of EMDR Practice and Research, 2(4), 247-261. https:// doi.org/10.1891/1933-3196.2.4.247

McGoldrick, T., Begum, M., \& Brown, K. W. (2008). EMDR and olfactory reference syndrome: A case series. Journal of EMDR Practice and Research, 2(1), 63-68. https://doi.org/10.1891/19333196.2.1.63

Meares, A. (1960). A system of medical hypnosis. The Julian Press.

Miller, E., \& Halpern, S. (1994). Letting go of stress. Inner Peace Music.

Mol, S. S. L., Arntz, A., Metsemakers, J. F. M., Dinant, G., Vilters-Van Montfort, P. A. P., \& Knottnerus, A. (2005). Symptoms of post-traumatic stress disorder after non-traumatic events: Evidence from an open population study. British Journal of Psychiatry, 186, 494-499. https://doi. org/10.1192/bjp.186.6.494

Nazari, H., Momeni, N., Jariani, M., \& Tarrahi, M. J. (2011). Comparison of eye movement desensitization and reprocessing with citalopram in treatment of obsessive-compulsive disorder. International Journal of Psychiatry in Clinical Practice, 15(4), 270-274. https://doi.org/10.3109/13651 501.2011 .590210 
Nicosia, G. J. (1995). A brief note: Eye movement desensitization and reprocessing is not hypnosis. Dissociation, 8(1), 69.

Nieuwenhuis, S., Elzinga, B. M., Ras, P. H., Berends, F., Duijs, P., Samara, Z., \& Slagter, H. A. (2013). Bilateral saccadic eye movements and tactile stimulation, but not auditory stimulation, enhance memory retrieval. Brain and Cognition, 81, 52-56. https://doi.org/10.1016/j.bandc.2012.10.003

Obradovic, J., Bush, N. R., Stamperdahl, J., Adler, N. E., \& Boyce, W. T. (2010). Biological sensitivity to context: The interactive effects of stress reactivity and family adversity on socioemotional behavior and school readiness. Child Development, 1, 270-289. https://doi.org/10.1111/j.14678624.2009.01394.x

Pace, P. (2003). Connecting ego states through time with EMDR and lifespan integration [Conference presentation]. Eighth EMDR International Association Conference, Denver, CO.

Pagani, M., Di Lorenzo, G., Monaco, L., Niolu, C., Siracusano, A., Verardo, A. R., Lauretti, G., Fernandez, I., Nicolais, G., Cogolo, P., \& Ammaniti, M. (2011). Pretreatment, intratreatment, and posttreatment EEG imaging of EMDR: Methodology and preliminary results from a single case. Journal of EMDR Practice and Research, 5(2), 42-56. https://doi.org/10.1891/19333196.5.2.42

Pagani, M., DiLorenzo, G., Verardo, A. R., Nicolais, G., Monaco, L., Lauretti, G., Russo, R., Niolu, C., Ammaniti, M. Fernandex, I., \& Siracusano, A. (2012). Neurobiological correlates of EMDR monitoring_an EEG study. PLoS ONE, 7(9), 1-12. https://doi.org/10.1371/journal.pone.0045753

Parker, A., Buckley, S., \& Dagnall, N. (2009). Reduced misinformation effects following saccadic bilateral eye movements. Brain and Cognition, 69, 89-97. https://doi.org/10.1016/j.bandc.2008.05.009

Parker, A., \& Dagnall, N. (2007). Effects of bilateral eye movements on gist based false recognition in the DRM paradigm. Brain and Cognition, 63, 221-225. https://doi.org/10.1016/j.bandc.2006.08.005

Parker, A., Relph, S., \& Dagnall, N. (2008). Effects of bilateral eye movement on retrieval of item, associative and contextual information. Neuropsychology, 22, 136-145. https://doi.org/10.1037/08944105.22.1.136

Patel, G. J., \& McDowall, J. (2016). The role of eye movements in EMDR: Conducting eye movements while concentrating on negative autobiographical memories results in fewer intrusions. Journal of EMDR Practice and Research, 10(1), 13-22. https://doi.org/10.1891/1933-3196.10.1.13

Perkins, B., \& Rouanzoin, C. (2002). A critical evaluation of current views regarding eye movement desensitization and reprocessing (EMDR): Clarifying points of confusion. Journal of Clinical Psychology, 58(1), 77-97. https://doi.org/10.1002/jclp.1130

Pitman, R. K., Orr, S. P., Altman, B., Longpre, R. E., Poire, R. E., \& Macklin, M. L. (1996). Emotional processing during eye movement desensitization and reprocessing therapy of Vietnam veterans with chronic posttraumatic stress disorder. Comprehensive Psychiatry, 37, 419-429. https://doi. org/10.1016/S0010-440X(96)90025-5

Propper, R. E., \& Christman, S. D. (2008). Interhemispheric interaction and saccadic horizontal eye movements: Implications for episodic memory, EMDR, and PTSD. Journal of EMDR Practice and Research, 2(4), 269-281. https://doi.org/10.1891/1933-3196.2.4.269.

Propper, R. E., Pierce, J., Geisler, M. W., Christman, S. D., \& Bellorado, N. (2007). Effect of bilateral eye movements on frontal interhemispheric gamma EEG coherence: Implications for EMDR therapy. Journal of Nervous Mental Disorders, 195(9), 785-788. https://doi.org/10.1097/ NMD.0b013e318142cf73

Raboni, M. R., Tufik, S., \& Suchecki, D. (2006). Treatment of PTSD by eye movement desensitization reprocessing (EMDR) improves sleep quality, quality of life, and perception of stress. Annals of the New York Academy of Sciences, 1071(1), 508-513. https://doi.org/10.1196/annals. 1364.054

Ray, A. L., \& Zbik, A. (2001). Cognitive behavioral therapies and beyond. In C. D. Tollison, J. R. Satterhwaite, \& J. W. Tollison (Eds.), Practical pain management (3rd ed., pp. 189-208). Lippincott.

Read, J., Fosse, R., Moskowitz, A., \& Perry, B. (2014). The traumagenic neurodevelopmental model of psychosis revisited. Neuropsychiatry, 4(1), 65-79. https://doi.org/10.2217/NPY.13.89 
Renfrey, G., \& Spates, C. R. (1994). Eye movement desensitization: A partial dismantling study. Journal of Behavior Therapy and Experimental Psychiatry, 25, 231-239. https://doi.org/10.1016/00057916(94)90023-X

Ricci, R. J., Clayton, C. A., \& Shapiro, F. (2006). Some effects of EMDR on previously abused child molesters: Theoretical reviews and preliminary findings. Journal of Forensic Psychiatry and Psychology, 17(4), 538-562. https://doi.org/10.1080/14789940601070431

Rimini, D., Molinari, F., Liboni, W., Balbo, M. Daro, R., Viotti, E., \& Fernandez, I. (2016). Effect of ocular movements during eye movement desensitization and reprocessing (EMDR) therapy: A near-infrared spectroscopy study. PlusOne, 11(10), e0164379. https://oi.org/10.1371/journal. pone. 0164379

Robinson, J. S., \& Larson, C. (2010). Are traumatic events necessary to elicit symptoms of posttraumatic stress? Psychological Trauma: Theory, Research, Practice, and Policy, 2, 71-76. https://doi. org/10.1037/a0018954

Rogers, S., \& Silver, S. M. (2002). Is EMDR an exposure therapy? A review of trauma protocols. Journal of Clinical Psychology, 58(1), 43-59. https://doi.org/10.1002/jclp.1128

Rogers, S., Silver, S. M., Goss, J., Obenchain, J., Willis, A., \& Whitney, R. L. (1999). A single session, group study of exposure and eye movement desensitization and reprocessing in treating posttraumatic stress disorder among Vietnam War veterans: Preliminary data. Journal of Anxiety Disorders, 13(1-2), 119-130. https://doi.org/10.1016/S0887-6185(98)00043-7

Russell, M. C. (2008). Treating traumatic amputation-related phantom limb pain: A case study utilizing eye movement desensitization and reprocessing within the Armed Services. Clinical Case Studies, 7(2), 136-153. https://doi.org/10.1177/1534650107306292

Russell, M. C., \& Figley, C. R. (2013). Treating traumatic stress injuries in military personnel: An EMDR practitioner's guide. Routledge Publishing.

Sabourin, M. E., Cutcomb, S. E., Crawford, H. J., \& Pribram, K. (1990). EEG correlates of hypnotic susceptibility and hypnotic trance: Spectral analysis and coherence. International Journal of Psychophysiology, 10, 125-142. https://doi.org/10.1016/0167-8760(90)90027-b

Sack, M., Hofmann, A., Wizelman, L., \& Lempa, W. (2008). Psychophysiological changes during EMDR and treatment outcome. Journal of EMDR Practice and Research, 2(4), 239-246. https:// doi.org/10.1891/1933-3196.2.4.239

Sack, M., Lempa, W., Steinmetz, A., Lamprecht, F., \& Hofmann, A. (2008). Alterations in autonomic tone during trauma exposure using eye movement desensitization and reprocessing (EMDR)Results of a preliminary investigation. Journal of Anxiety Disorders, 22(7), 1264-1271. https:// doi.org/10.1016/j.janxdis.2008.01.007

Sack, M., Zehl, S., Otti, A., Lahmann, C., Henningsen, P., Kruse, J., \& Stingl, M. (2016). A comparison of dual attention, eye movements, and exposure only during eye movement desensitization and reprocessing for posttraumatic stress disorder: Results from a randomized clinical trial. Psychotherapy and Psychosomatics, 85(6), 357-365. https://doi.org/10.1159/000447671

Samara, Z., Bernet, M., Elzinga, B. M., Heleen, A., Slagter, H. A., \& Nieuwenhuis, S. (2011). Do horizontal saccadic eye movements increase interhemispheric coherence? Investigation of a hypothesized neural mechanism underlying EMDR. Frontiers in Psychiatry, 2, 4. https://doi. org/10.3389/fpsyt.2011.00004

Sanderson, A., \& Carpenter, R. (1992). Eye movement desensitization versus image confrontation: A single-session crossover study of 58 phobic subjects. Journal of Behavior Therapy and Experimental Psychiatry, 23, 269-275. https://doi.org/10.1016/0005-7916(92)90049-o

Schneider, J., Hofmann, A., Rost, C., \& Shapiro, F. (2007). EMDR and phantom limb pain: Theoretical implications, case study, and treatment guidelines. Journal of EMDR Practice and Research, 1(1), 31-45. https://doi.org/10.1891/1933-3196.1.1.31

Schneider, J., Hofmann, A., Rost, C., \& Shapiro, F. (2008). EMDR in the treatment of chronic phantom limb pain. Pain Medicine, 9(1), 76-82. https://doi.org/10.1111/j.15264637.2007.00299.x 
Schubert, S. J., Lee, C. W., \& Drummond, P. D. (2011). The efficacy and psychophysiological correlates of dual-attention tasks in eye movement desensitization and reprocessing (EMDR). Journal of Anxiety Disorders, 25, 1-11. https://doi.org/10.1016/j.janxdis.2010.06.024

Servan-Schreiber, D., Schooler, J., Dew, M. A., Carter, C., \& Bartone, P. (2006). Eye movement desensitization and reprocessing for posttraumatic stress disorder: A pilot blinded, randomized study of stimulation type. Psychotherapy and Psychosomatics, 75(5), 290-297. https://doi. org/10.1159/000093950

Shapiro, E., \& Laub, B. (2014). The recent traumatic episode protocol (R-TEP): An integrative protocol for early EMDR intervention (EEI). In M. Luber (Ed.), Implementing EMDR early mental health interventions for man-made and natural disasters: Models, scripted protocols, and summary sheets (pp. 193-207). Springer Publishing Company.

Shapiro, F. (1989a). Efficacy of the eye movement desensitization procedure in the treatment of traumatic memories. Journal of Traumatic Stress, 2(2), 199-223. https://doi.org/10.1007/ BF00974159

Shapiro, F. (1989b). Eye movement desensitization: A new treatment for post-traumatic stress disorder. Journal of Behavior Therapy and Experimental Psychiatry, 20(3), 211-217. https://doi. org/10.1016/0005-7916(89)90025-6

Shapiro, F. (1995). Eye movement desensitization and reprocessing: Basic principles, protocols and procedures (1st ed.). Guilford Press.

Shapiro, F. (2001). Eye movement desensitization and reprocessing: Basic principles, protocols and procedures (2nd ed.). Guilford Press.

Shapiro, F. (2002). EMDR as an integrative psychotherapy approach: Experts of diverse orientations explore the paradigm prism. American Psychological Association Press.

Shapiro, F. (2006). EMDR: New notes on adaptive information processing with case formulations principles, forms, scripts and worksheets, version 1.1. EMDR Institute.

Shapiro, F. (2007a). EMDR, adaptive information processing, and case conceptualization. Journal of EMDR Practice and Research, 1(2), 68-87. https://doi.org/10.1891/1933-3196.1.2.68

Shapiro, F. (2007b). EMDR and case conceptualization from an adaptive information processing perspective. In F. Shapiro, F. Kaslow, \& L. Maxfield (Eds.), Handbook of EMDR and family therapy processes (pp. 3-36). John Wiley.

Shapiro, F. (2009-2017a). The EMDR approach to psychotherapy-EMDR Institute basic training course: Weekend 1 of the two part basic training. EMDR Institute.

Shapiro, F. (2009-2017b). The EMDR approach to psychotherapy-EMDR Institute basic training course: Weekend 2 of the two part basic training. EMDR Institute.

Shapiro, F. (2012a). EMDR therapy: An overview of current and future research. European Review of Applied Psychology, 62(4), 193-195. https://doi.org/10.1016/j.erap.2012.09.005

Shapiro, F. (2012b). Getting past your past: Take control of your life with self-help techniques from EMDR therapy. Rodale Books.

Shapiro, F. (2014). The role of eye movement desensitization and reprocessing (EMDR) therapy in medicine: Addressing the psychological and physical symptoms stemming from adverse life experience. Permanente Journal, 18(1), 71-77. https://doi.org/10.7812/TPP/13-098

Shapiro, F. (2018). Eye movement desensitization and reprocessing: Basic principles, protocols and procedures (3rd ed.). Guilford Press.

Shapiro, F., \& Forrest, M. S. (2016). EMDR: The breakthrough therapy for overcoming anxiety, stress, and trauma (2nd ed.). Basic Books.

Shapiro, F., Kaslow, F. W., \& Maxfield, M. (2007). Handbook of EMDR and family therapy processes. John Wiley.

Sharpley, C. F., Montgomery, I. M., \& Scalzo, L. (1996). Comparative efficacy of EMDR and alternative procedures in reducing the vividness of mental images. Scandinavian Journal of Behaviour Therapy, 25, 37-42. https://doi.org/10.1080/16506079609456006

Smeets, M. A. M., Dijs, M. W., Pervan, I., Engelhard, I. M., \& van den Hout, M. (2012). Time-course of eye movement-related decrease in vividness and emotionality of unpleasant autobiographical memories. Memory, 20(4), 346-357. https://doi.org/10.1080/09658211.2012.665462 
Solomon, R. M., \& Shapiro, F. (2008). EMDR and the adaptive information processing model: Potential mechanisms of change. Journal of EMDR Practice and Research, 2(4), 315-325. https://doi. org/10.1891/1933-3196.2.4.315

Solomon, S. D., Gerrity, E. T., \& Muff, A. M. (1992). Efficacy of treatments for posttraumatic stress disorder: An empirical review. Journal of the American Medical Association, 268, 633-638. https:// doi.org/10.1001/jama.1992.03490050081031

Spates, C. R., Koch, E., Cusack, K., Pagoto, S., \& Waller, S. (2009). Eye movement desensitization and reprocessing. In E. B. Foa, T. M. Keane, M. J. Friedman, \& J. A. Cohen (Eds.), Effective treatments for PTSD: Practice guidelines from the International Society for Traumatic Stress Studies (2nd ed., pp. 279-305). Guilford Press.

Stickgold, R. (2002). EMDR: A putative neurobiological mechanism of action. Journal of Clinical Psychology, 58(1), 61-75. https://doi.org/10.1002/jclp.1129

Stickgold, R. (2008). Sleep-dependent memory processing and EMDR action. Journal of EMDR Practice and Research, 2(4), 289-299. https://doi.org/10.1891/1933-3196.2.4.289

Suzuki, A., Josselyn, S. A., Frankland, P. W., Masushige, S., Silva, A. J., \& Satoshi, K. (2004). Memory reconsolidation and extinction have distinct temporal and biochemical signatures. Journal of Neuroscience, 24(20), 4787-4795. https://doi.org/10.1523/jneurosci.5491-03.2004

Teicher, M. H., Samson, J. A., Sheu, Y.-S., Polcari, A., \& McGreenery, C. E. (2010). Hurtful words: Association of exposure to peer verbal abuse with elevated psychiatric symptom scores and corpus callosum abnormalities. American Journal of Psychiatry, 167, 1464-1471. https://doi.org/10.1176/ appi.ajp.2010.10010030

Uribe, M. E. R., \& Ramirez, E. O. L. (2006). The effect of EMDR therapy on the negative information processing on patients who suffer depression. Revista Electrónica de Motivación y Emoción, 9, 23-24.

van den Berg, D. P. G., \& van der Gaag, M. (2012). Treating trauma in psychosis with EMDR: A pilot study. Journal of Behavior Therapy and Experimental Psychiatry, 43(1), 664-671. https:// doi.org/10.1016/j.jbtep.2011.09.011

van den Hout, M. A., Engelhard, I. M., Rijkeboer, M. M., Koekebakker, J., Hornsveld, H., Leer, A., Toffolo, M. B. J., Akse, N. (2011). EMDR: Eye movements superior to beeps in taxing working memory and reducing vividness of recollections. Behaviour Research and Therapy, 49, 92-98. https://doi.org/10.1016/j.brat.2010.11.003

van den Hout, M. A., \& Engelhard, I. (2012). How does EMDR work? Journal of Experimental Psychopathology, 3, 724-738. https://doi.org/10.5127/jep.028212

van den Hout, M. A., Engelhard, I. M., Smeets, M. A., Hornsveld, H., Hoogeveen, E., and de Heer, E. (2010). Counting during recall: Taxing of working memory and reduced vividness and emotionality of negative memories. Applied Cognitive Psychology, 24(3), 303-311. https://doi. org/10.1002/acp.1677

van den Hout, M. A., Muris, P., Salemink, E., \& Kindt, M. (2001). Autobiographical memories become less vivid and emotional after eye movements. British Journal of Clinical Psychology, 40(2), 121-130. https://doi.org/10.1348/014466501163535

van den Hout, M. A., Rijkeboer, M. M., Engelhard, I. M., Klugkist, I., Hornsveld, H., Toffolo, M. J., \& Cath, D. C. (2012). Tones inferior to eye movements in the EMDR treatment of PTSD. Behaviour Research and Therapy, 50(5), 275-279. https://doi.org/10.1016/j.brat.2012.02.001

van der Kolk, B. A., Spinazzola, J., Blaustein, M. E., Hopper, J. W., Hopper, E. K., Korn, D. L., \& Simpson, W. B. (2007). Randomized clinical trial of eye movement desensitization and reprocessing (EMDR), fluoxetine, and pill placebo in the treatment of posttraumatic stress disorder: Treatment effects and long-term maintenance. Journal of Clinical Psychiatry, 68(1), 37-46. https://doi. org/10.4088/JCP.v68n0105

van Etten, M. L., \& Taylor, S. (1998). Comparative efficacy of treatments for posttraumatic stress disorder: A meta-analysis. Clinical Psychology \& Psychotherapy, 5, 126-144. https://doi.org/10.1002/ (SICI)1099-0879(199809)

van Schie, K., Engelhard, I. M., Klugkist, I., \& van den Hout, M. A. (2016). Blurring emotional memories using eye movements: Individual differences and speed of eye movements. European Journal of Psychotraumatology, 7, 29476. https://doi.org/10.3402/ejpt.v7.29476 
van Veen, S. C., Engelhard, I. M., \& van den Hout, M. A. (2016). The effects of eye movements on emotional memories: using an objective measure of cognitive load. European Journal of Psychotraumatology, 7, 1-11. https://doi.org/10.3402/ejpt.v7.30122

van Veen, S. C., van Schie, K., Wijngaards-de Meij, L. D., Littel, M., Engelhard, I. M., \& van den Hout, M. A. (2015). Speed matters: Relationship between speed of eye movements and modification of aversive autobiographical memories. Frontiers in Psychiatry, 6(45), 1-9. https://doi.org/10.3389/ fpsyt.2015.00045

Varese, F., Smeets, F., Drukker, M., Lieverse, R., Lataster, T., Viechtbauer, W., Read, J., van Os, J., Bentall, R. P. (2012). Childhood adversities increase the risk of psychosis: A meta-analysis of patient-control, prospective- and cross-sectional cohort studies. Schizophrenia Bulletin, 38(4), 661-671. https://doi.org/10.1093/schbul/sbs050

Wesselmann, D., \& Potter, A. E. (2009). Change in adult attachment status following treatment with EMDR: Three case studies. Journal of EMDR Practice and Research, 3(3), 178-191. https://doi. org/10.1891/1933-3196.3.3.178

Wilensky, M. (2006). Eye movement desensitization and reprocessing (EMDR) as a treatment for phantom limb pain. Journal of Brief Therapy, 5(1), 31-44. https://doi.org/10.1186/1471-2474-14-256

Wilson, D., Silver, S. M., Covi, W., \& Foster, S. (1996). Eye movement desensitization and reprocessing: Effectiveness and autonomic correlates. Journal of Behavior Therapy and Experimental Psychiatry, 27, 219-229. https://doi.org/10.1016/S0005-7916(96)00026-2

Wolpe, J. (1990). The practice of behavior therapy (4th ed.). Pergamon Press.

Zarghi, A. (2015). Memory editing with emphasizing the role of EM in EMDR. International Clinical Neuroscience Journal, 2(2), 66-70. https://doi.org/10.22037/icnj.v2i2.9473 